TRANSACTIONS OF THE

AMERICAN MATHEMATICAL SOCIETY

Volume 351, Number 3, March 1999, Pages 901-945

S $0002-9947(99) 02351-X$

\title{
ERRATIC SOLUTIONS OF SIMPLE DELAY EQUATIONS
}

\author{
BERNHARD LANI-WAYDA
}

\begin{abstract}
We give an example of a smooth function $g: \mathbb{R} \longrightarrow \mathbb{R}$ with only one extremum, with sign $g(x)=-\operatorname{sign} g(-x)$ for $x \neq 0$, and the following properties: The delay equation $\dot{x}(t)=g(x(t-1))$ has an unstable periodic solution and a solution with phase curve transversally homoclinic to the orbit of the periodic solution.

The complicated motion arising from this structure, and its robustness under perturbation of $g$, are described in terms of a Poincaré map. The example is minimal in the sense that the condition $g^{\prime}<0$ (under which there would be no extremum) excludes complex solution behavior.

Based on numerical observations, we discuss the role of the erratic solutions in the set of all solutions.
\end{abstract}

\section{INTRODUCTION}

In this work we try to find and describe complicated solution behavior in a functional differential equation as simple as possible. Let us introduce the framework for the class of equations that we consider. Let $E, F$ be normed spaces, and $M \subset E$ be an open subset. For $k \in \mathbb{N}_{0}$, the space of bounded $C^{k}$-maps $f: M \rightarrow F$ with bounded derivatives up to order $k$ is denoted by $B C^{k}(M, F)$. The norm on this space is given by

$$
|f|_{C^{k}}=\max _{j=0, \ldots, k} \sup _{x \in M}\left|D^{j} f(x)\right|,
$$

with the usual norm on $L_{c}(E, F)$. (The space $B C^{k}(M, F)$ is a Banach space if $F$ is a Banach space.) For compact intervals $I \subset \mathbb{R}$, the space $C^{k}(I, F)$ is defined analogously. When convenient, we write

$$
\left(B C^{k}(M, F),||_{C^{k}}\right) \quad \text { and }\left(C^{k}(I, F),||_{C^{k}}\right) .
$$

Let $C:=C^{0}([-1,0], \mathbb{R})$. If $I \subset \mathbb{R}$ is an interval, $t \in \mathbb{R},[t-1, t] \subset I$ and $x: I \longrightarrow$ $\mathbb{R}$ is continuous, the segment $x_{t} \in C$ is defined by $x_{t}(s):=x(t+s)(s \in[-1,0])$.

If $G \in B C^{1}(C, \mathbb{R})$ and $\varphi \in C$, the initial value problem

$$
\left\{\begin{aligned}
\dot{x}(t) & =G\left(x_{t}\right) \\
x_{0} & =\varphi
\end{aligned}\right.
$$

has a unique solution $x^{\varphi, G}:[-1, \infty) \longrightarrow \mathbb{R}$ (see [HV-L] or [DvGV-LW]). The map $\Phi^{G}: \mathbb{R}_{0}^{+} \times C \longrightarrow C, \quad(t, \varphi) \mapsto x_{t}^{\varphi, G}$ is a continuous semiflow. The restriction

Received by the editors September 4, 1996.

1991 Mathematics Subject Classification. Primary 34K15, 58F13, 70K50.

Supported by the Deutsche Forschungsgemeinschaft within the Schwerpunkt Analysis, Ergodentheorie und Effiziente Simulation Dynamischer Systeme. 
$\Phi^{G} \mid(1, \infty) \times C$ is $C^{1}$, and $D_{2} \Phi^{G}$ exists on $\mathbb{R}_{0}^{+} \times C$. See [L-W1] for a proof of smoothness properties, also with respect to $G$.

If $g: \mathbb{R} \longrightarrow \mathbb{R}$ and $G(\psi)=g(\psi(-1))$ for all $\psi \in C$, then the functional differential equation

$$
\dot{x}(t)=G\left(x_{t}\right)
$$

is equivalent to

$$
\dot{x}(t)=g(x(t-1)) .
$$

We also write $\Phi^{g}$ for the semiflow $\Phi^{G}$ in this case. It is easy to see that if $g \in$ $B C^{1}(\mathbb{R}, \mathbb{R})$, then $G \in B C^{1}(C, \mathbb{R})$, and $D G(\psi) \chi=g^{\prime}(\psi(-1)) \chi(-1)$ for $\psi, \chi \in C$.

Note that, if $G(\psi)=-\mu \psi(0)+g(\psi(-1))$, then eq. $(G)$ reads as

$$
\dot{x}(t)=-\mu x(t)+g(x(t-1)) .
$$

Functional differential equations of type $(G)$ are used as models in engineering and biology; see, e.g., [Dr, Chapter V].

Complicated motion. Numerical solutions that appear to be 'chaotic' have been observed in a number of experiments; among the earliest are the works of Mackey and Glass [MG], Lasota [La] and of Lasota and Wazewska-Czyzewska [LWCz] on models of type $(\mu, g)$ for physiological control processes with delayed negative feedback.

See [GP] for a computation of dimension-like quantities from numerical trajectories of the Mackey-Glass equation.

If 'chaos' is to be described in mathematical terms, the first question is how to render an analytical notion of 'chaotic' behavior. One way of doing this is to demand the existence of a subset of the state space on which the dynamics is equivalent to the index shift on a space of symbol sequences indexed with $\mathbb{Z}$. Since the work of Morse and Hedlund $[\mathrm{M}],[\mathrm{MH}]$, the symbol shift is a standard model for irregular, quasi-random motion. Typically one extracts a Poincaré map $\Pi$ from the semiflow and proves that trajectories $\left(x_{n}\right)_{n \in \mathbb{Z}}$ of $\Pi$ in some open set $W$ can be described by symbol sequences $\left(a_{n}\right)_{n \in \mathbb{Z}}$. The value of $a_{n}$ indicates in which of several disjoint subsets of $W$ the point $x_{n}$ is contained. The existence of such a shift embedding follows if $\Pi$ has a hyperbolic fixed point and a trajectory transversally homoclinic to that fixed point. For diffeomorphisms in finite dimension, this result is due to Smale [S]. A shadowing lemma approach that yields a description of trajectories in some neighborhood of the homoclinic orbit was presented by Palmer $[\mathrm{P}]$ and by Kirchgraber and Stoffer [KS]. For noninvertible mappings in infinite dimension, corresponding results which even give a complete symbolic coding of all trajectories in some neighborhood were proved by Hale and Lin [HL1], and, using a generalized shadowing lemma, by Steinlein and Walther [SW].

The 'transversally homoclinic' framework applies to all presently known examples of delay equations for which complicated motion is analytically proven. For the smoothed step functions from the earliest examples by Walther [W1] and An der Heiden and Walther [AdHW], complicated orbits were first constructed by embedding an interval map into a Poincaré map for the semiflow; the interval map had properties as in the paper by Li and Yorke [LY]. Later, Hale and Lin [HL2] remarked that these examples fit into the setting of transversally homoclinic points for a Poincaré map. A detailed proof of this statement and a robustness result 
were given in [L-W1]. In the more recent examples from [W2] and [L-WW2], the approach was from the beginning to construct transversally homoclinic points.

Hale and Sternberg [HS] obtained numerical evidence for the occurrence of homoclinic orbits in the Mackey-Glass equation.

Let us briefly comment on the scope and the limitations of shift embedding results in the 'transversally homoclinic' setting. On the one hand, the trajectories described by symbol sequences are robust under $C^{1}$-perturbations of the equation (see [L-W1] and [L-WW2]). In this sense, the presence of shift dynamics is an essential feature of the dynamical system.

On the other hand, the points on the 'chaotic' trajectories comprise only a 'thin' (Cantor) set, and these trajectories are dynamically unstable. It may well be that a typical solution does not spend much time in the vicinity of the special solutions captured by the symbolic coding. In fact, the numerical observations reported in Section 7 suggest that this may be the case for examples similar to the one constructed in the present paper. Results that capture complicated behavior on 'large' subsets of phase space, for dynamical systems given by simple analytical expressions, are not easy to obtain even in low finite dimensions. (Compare, e.g., $[\mathrm{BC} 1],[\mathrm{BC} 2]$ and $[\mathrm{Laz}]$.$) The observations from [DL-W] suggest that, numerically,$ erratic motion can take place for every initial value. A corresponding analytical result for delay equations is currently not available.

Monotone nonlinearities. If $g(0)=0$, the monotonicity condition

$$
g^{\prime}<0
$$

excludes erratic solutions, according to the Poincaré-Bendixson theorem for delay equations proved by Mallet-Paret and Sell [M-PS]. It was shown by Walther [W3] that, under condition $(\mathrm{M})$, equation $(\mu, g)$ with $\mu \geq 0$ has a global two-dimensional attractor homeomorphic to a disc, and the planar dynamics on this attractor cannot be complicated. Define the set $S$ of data with at most one change of sign by

$$
\begin{gathered}
S=\{\varphi \in C \backslash\{0\}: \text { There exist } a \in[-1,0] \text { and } j \in\{0,1\} \text { with } \\
0 \leq(-1)^{j} \varphi(t) \text { for } t \leq a \\
\text { and } \left.(-1)^{j} \varphi(t) \leq 0 \text { for } a \leq t\right\} .
\end{gathered}
$$

Functions $x$ defined on an interval unbounded to the right are called eventually slowly oscillating if $x_{t} \in S$ holds for all sufficiently large $t$. Further, $x: \mathbb{R} \longrightarrow \mathbb{R}$ is called slowly oscillating if $x_{t} \in S$ for all $t \in \mathbb{R}$. The set $S$ is of dominant importance for the semiflow induced by equation $(g)$, if condition $(\mathrm{M})$ holds: $S$ is invariant, and it is shown in [M-PW] that the domain of attraction into $S$ is open and dense in $C$.

Negative feedback. Consider now the negative feedback condition

$$
x \cdot g(x)<0 \text { for } x \neq 0,
$$

which is weaker than (M). The set $S$ is also invariant if only condition (NF) holds, and the domain of attraction into $S$ is open. Density of this domain in $C$ is not yet proved, but likely. Condition (NF) still has a restrictive effect on the possible complexity of the semiflow: It was shown by Mallet-Paret [M-P] that under (NF), the set of all bounded solutions which are defined on all of $\mathbb{R}$ admits a Morse decomposition. This is a partition into $N$ invariant subsets $S_{1}, \ldots, S_{N}$, and into 
solutions that are asymptotic to some $S_{j}$ for $t \longrightarrow-\infty$ and to some $S_{k}$ with $k<j$ for $t \longrightarrow \infty$. The index $i \in\{1, \ldots, N\}$ describes the number of zeroes per time unit, and the solutions in the 'lowest' Morse set $S_{1}$ are the slowly oscillating solutions. The Morse decomposition result means that, if the behavior of the solutions inside each $S_{j}$ is ignored, the orbit structure looks like the attractor of a gradient flow - equilibria and connecting orbits. However, it was shown in [L-WW2] that (NF) does not exclude complicated motion, and that erratic solutions of $(\mathrm{g})$ can be found within the slowly oscillating class. The example function $g$ from [L-WW2] has a rather complicated shape with at least two extrema.

A minimal example with erratic solutions. The purpose of the present paper is to construct smooth functions $g$ such that equation $(g)$ admits a shift embedding (and hence has a set of erratic solutions), and with an only 'minimal' violation of condition (M): $g$ satisfies (NF) and has only one extremum. We obtain a solution transversally homoclinic to an unstable periodic solution. The homoclinic solution has a simpler shape, compared to the previous example [L-WW2]. The main result is formulated in Theorem 6.1.

Let us mention the related result of Gedeon $[\mathrm{G}]$ for ordinary differential equations with a cyclic feedback structure: That example also has an only minimal violation of monotonicity conditions that would exclude chaotic solutions, according to the result of Mallet-Paret and Smith [M-PSm]. Transversality is not proved in [G], but even without transversality, a semiconjugacy with a symbol shift can be proved.

The techniques of proof in the present paper are based on [L-WW2], with some improvements. In particular, one important step is to achieve that a solution $z$ starting from an initial value $\varphi$ in the unstable manifold of the periodic orbit is close to a prescribed 'target' $\psi$ at some time $t^{*}$; an estimate of the form $\left|z_{t^{*}}-\psi\right| \leq \epsilon$ is obtained. The idea of the construction enforces that $\varphi$ is outside and $\psi$ inside some neighborhood of the periodic orbit $\mathcal{O}$. Two technical improvements, compared to [L-WW2], make the simpler shape of $g$ and of the homoclinic solution possible:

1) The relation between the distances of $\varphi$ and $\psi$ to $\mathcal{O}$ is expressed in a more precise, quantitative way.

2) The method of estimating $\left|z_{t^{*}}-\psi\right|$ uses also second order terms of Taylor expansions.

We obtain the homoclinic solution from a shooting-type argument. The transversality proof is based on the criterion from Theorem 8.2 of [L-WW1]. The latter result characterizes transversality by the condition that certain solutions of the variational equation along the homoclinic solution are eventually slowly oscillating. That this condition can be verified is enforced by suitable modifications of the nonlinearity.

Although the so far simplest example of delay equations with transversally homoclinic solutions, our nonlinearity is still an 'artificial' construction, not given by a simple expression. The erratic solutions, which are analytically described, will be hard to observe in numerical experiments, as is discussed in Section 7.

\section{Homoclinic solutions, Poincaré maps and transversality}

In this section, we set up a framework for the analytic description of erratic solutions of functional differential equations. Symbolic coding of the solutions, 
and their robustness under perturbation of the equation, is expressed in terms of associated Poincaré maps.

A sequence $\left(\chi_{n}\right)_{n \in \mathbb{Z}}$ of points from the domain of a map $P$ is called a trajectory of $P$ if $P\left(\chi_{n}\right)=\chi_{n+1} \quad(n \in \mathbb{Z})$.

Definition 2.1. Let $X$ be a Banach space, $H \subset X$ a closed hyperplane, and $\Phi: \mathbb{R}_{0}^{+} \times X \longrightarrow X$ a semiflow. Let $U \subset C$ and $\varphi \in U$. A map $P: U \longrightarrow H$ is called a Poincaré map for $\Phi$ if the following conditions hold.

(i) There exist an open interval $I$ with $\inf I>0$ and a map $\tau: U \longrightarrow I$ with the property

$$
\forall t \in I \forall \psi \in U: \quad \Phi(t, \psi) \in H \Longleftrightarrow t=\tau(\psi) .
$$

(ii) $P(\psi)=\Phi(\tau(\psi), \psi))$ for all $\psi \in U$.

Definition 2.2. If $n \in \mathbb{N}$ and $H_{i} \subset X$ are hyperplanes, and $U_{i} \subset X$ are pairwise disjoint, and $P_{i}: U_{i} \longrightarrow H_{i}, \quad \psi \mapsto \Phi\left(\tau_{i}(\psi), \psi\right)$ are Poincaré maps as in Definition $2.1(i=1, \ldots, n)$, then the map

$$
\Pi: \bigcup_{i=1}^{n} U_{i} \longrightarrow \bigcup_{i=1}^{n} H_{i}, \quad \Pi(\psi):=P_{i}(\psi) \text { if } \psi \in U_{i}
$$

is also called a Poincaré map.

Remark 2.3. If $\Pi: \bigcup_{i=1}^{n} U_{i} \longrightarrow \bigcup_{i=1}^{n} H_{i}$ is a Poincaré map, then every trajectory $\left(\chi_{k}\right)_{k \in \mathbb{Z}}$ of $\Pi$ defines a function $x: \mathbb{R} \longrightarrow X$ with the properties

(i) $x(t+s)=\Phi(s, x(t))$ for $t \in \mathbb{R}, s \geq 0$;

(ii) there is a sequence $\left(t_{k}\right)_{k \in \mathbb{Z}}$ with $x\left(t_{k}\right)=\chi_{k}(k \in \mathbb{Z})$.

Proof. For every $k \in \mathbb{Z}$ there exists a unique $i(k) \in\{1, \ldots, n\}$ with $\chi_{k} \in U_{i(k)}$ and $\Pi\left(\chi_{k}\right)=\Phi\left(\tau_{i(k)}\left(\chi_{k}\right), \chi_{k}\right)$. Define $t_{0}:=0, t_{k+1}:=t_{k}+\tau_{i(k)}\left(\chi_{k}\right)$ for $k \geq 0$, and $t_{k-1}:=t_{k}-\tau_{i(k-1)}\left(\chi_{k-1}\right)$ for $k \leq 0$. Since $\tau_{i}$ maps into an interval $I_{i}$ with $\inf I_{i}>$ $0(i=1, \ldots, n)$, we have $\sum_{k=0}^{n} t_{ \pm k} \longrightarrow \pm \infty$ for $n \longrightarrow \infty$. Define $x: \mathbb{R} \longrightarrow \mathbb{R}$ by $x(t):=\Phi\left(t-t_{k-1}, \chi_{k-1}\right)$ if $t \in\left[t_{k-1}, t_{k}\right), k \in \mathbb{Z}$. Then property (ii) is satisfied. Let $t \in \mathbb{R}, s \geq 0$. There exist uniquely determined $k, l \in \mathbb{Z}$ with $k \leq l$, with $t \in\left[t_{k-1}, t_{k}\right)$ and $t+s \in\left[t_{l-1}, t_{l}\right)$. Then

$$
\begin{aligned}
x(t+s) & =\Phi\left(t+s-t_{l-1}, \chi_{l-1}\right)=\Phi\left(t+s-t_{l-1}, \Pi^{l-1-(k-1)} \chi_{k-1}\right) \\
& =\Phi\left(t+s-t_{l-1}, \Phi\left(\sum_{j=k-1}^{l-2} \tau_{i(j)}\left(\chi_{j}\right), \chi_{k-1}\right)\right) \\
& =\Phi\left(t+s-t_{l-1}, \Phi\left(\sum_{j=k-1}^{l-2} t_{j+1}-t_{j}, \chi_{k-1}\right)\right)
\end{aligned}
$$

(From the definition of the $t_{j}$;

in case $k=l$, the sum is to be read as zero.)

$$
\begin{aligned}
& =\Phi\left(t+s-t_{l-1}, \Phi\left(t_{l-1}-t_{k-1}, \chi_{k-1}\right)\right)=\Phi\left(s, \Phi\left(t-t_{k-1}, \chi_{k-1}\right)\right) \\
& =\Phi(s, x(t)) .
\end{aligned}
$$

Let now $H \subset X$ be a hyperplane. There exists a continuous linear functional $h \in X^{*}$ and $c \in \mathbb{R}$ such that $H=h^{-1}(\{c\})$. With $H_{0}:=\operatorname{ker} h$, one has $H=\varphi+H_{0}$ 
for every $\varphi \in H$. Note that so far we have not required any smoothness properties of $\tau$ and $P$. If $I \subset \mathbb{R}^{+}$is open and $\Phi$ is $C^{1}$ on $I \times X$, then smooth Poincaré maps are typically obtained from the Implicit Function Theorem, if the conditions $\Phi\left(t^{*}, \varphi\right) \in H$ and $D_{1} \Phi\left(t^{*}, \varphi\right) 1 \notin H_{0}$ are satisfied (see, e.g., [L-W1], or Section XIV.3 of $[\mathrm{DvGV}-\mathrm{LW}])$.

Poincaré maps associated with functional differential equations of type $(G)$ can be described as functions not only of the initial value $\varphi \in C$ but also of the nonlinearity $G$. The following theorem is a slight variant of Theorem 1.7 from $[\mathrm{L}-\mathrm{W} 1]$. For $F \in B C^{1}(C, \mathbb{R})$, we denote by $\Phi(\cdot, \cdot, F): \mathbb{R}_{0}^{+} \times C \longrightarrow C$ the semiflow generated by equation $(F)$. It follows from Lemma 1.5 of [L-W1] that the $\operatorname{map} \Phi: \mathbb{R}_{0}^{+} \times C \times\left(B C^{1}(C, \mathbb{R}),||_{C^{1}}\right) \longrightarrow C$ is continuous, and its restriction to $(1, \infty) \times C \times B C^{1}(C, \mathbb{R})$ is $C^{1}$.

Theorem 2.4. Let $h \in C^{*}$ be a continuous linear functional, $c \in \mathbb{R}$, and set $H:=h^{-1}(\{c\}) \subset C$. Assume $t_{0}>1$, that $\varphi \in C, G \in B C^{1}(C, \mathbb{R})$ and $x_{t_{0}}^{\varphi, G} \in$ $H, h\left(\dot{x}_{t_{0}}^{\varphi, G}\right) \neq 0$. Let $\mathcal{U} \subset C$ be an open neighborhood of the $\operatorname{set}\left\{x_{s}^{\varphi, G}: 0 \leq s \leq\right.$ $\left.t_{0}\right\}$.

Then there exist bounded open neighborhoods $U \subset C$ of $\varphi$ and $\mathcal{B} \subset B C^{1}(C, \mathbb{R})$ of $G$ and a bounded $C^{1}$ map $\tau: U \times \mathcal{B} \longrightarrow \mathbb{R}$ with the following properties.

(i) For $F \in \mathcal{B}$, the map $P_{F}: U \longrightarrow H \subset C, \psi \mapsto \Phi(\tau(\psi, F), \psi, F)$ is a Poincaré map for $\Phi(\cdot, \cdot, F)$, and $P_{F} \in B C^{1}(U, C)$. Further, $\tau(\varphi, G)=t_{0}$, so that $P_{G}(\varphi)=\Phi\left(t_{0}, \varphi, G\right)$.

(ii) For $\psi \in U, F \in \mathcal{B}$ and $s \in[0, \tau(\psi, F)]$ one has $\Phi(s, \psi, F) \in \mathcal{U}$.

(iii) If $\tilde{G} \in \mathcal{B}$ and $D \tilde{G}_{\mid \mathcal{U}}$ is uniformly continuous on $\mathcal{U}$, then $D P_{\tilde{G}}$ is uniformly continuous, and the map $\mathcal{B} \ni F \mapsto P_{F} \in\left(B C^{1}(U, C),\left.\right|_{C^{1}}\right)$ is continuous at $\tilde{G}$.

Proof. There exists $T>t_{0}$ such that $\left\{x_{s}^{\varphi, G}: s \in[0, T]\right\} \subset \mathcal{U}$. The conditions of Theorem 1.7 from [L-W1] are satisfied by $\varphi, \mathcal{U}$ and $G_{\mid \mathcal{U}}$. From the latter theorem and from Lemma 1.5 of the same reference, we obtain neighborhoods $U$ of $\varphi$ in $C$ and $\tilde{\mathcal{B}}$ of $G_{\mid \mathcal{U}}$ in $B C^{1}(\mathcal{U}, \mathbb{R})$ and a local semiflow $\tilde{\Phi}:[0, T] \times U \times \tilde{\mathcal{B}} \longrightarrow \mathcal{U} \subset C$. The set $\mathcal{B}:=\left\{F \in B C^{1}(C, \mathbb{R}): F_{\mid \mathcal{U}} \in \tilde{\mathcal{B}}\right\}$ is an open neighborhood of $G$ in $B C^{1}(C, \mathbb{R})$. It is clear that $\tilde{\Phi}\left(\cdot, \cdot, F_{\mid \mathcal{U}}\right)$ is a restriction of $\Phi(\cdot, \cdot, F)$ for $F \in \mathcal{B}$ (compare Remark 1.6 from [L-W1]). The assertions now follow from Theorem 1.7 of [L-W1].

We want to describe erratic solutions of delay equations by 'chaotic' trajectories of a Poincaré map $\Pi$. Such trajectories exist in a neighborhood of a transversally homoclinic orbit of $\Pi$. We recall this notion, and we assume the reader to be familiar with local invariant manifolds at a hyperbolic fixed point.

Definition 2.5. Let $U$ be an open subset of the Banach space $X$, and let $\Pi: U \longrightarrow$ $X$ be a $C^{1}$ map. Let $z \in U$ be a hyperbolic fixed point of $\Pi$ and let $W^{u}, W^{s}$ be local unstable and stable manifolds of $\Pi$ at $z$, respectively. A trajectory $\left(x_{n}\right)_{n \in \mathbb{Z}}$ of $\Pi$ is called a transversally homoclinic trajectory (or orbit) if the following condition is satisfied:

There exists $n_{0} \in \mathbb{N}$ such that for $m, n \in \mathbb{Z}, n \geq n_{0}, m \leq-n_{0}$, one has $x_{m} \in$ $W^{u}, x_{n} \in W^{s}$, and $D f^{n-m}\left(x_{m}\right)$ maps the tangent space $T_{x_{m}} W^{u}$ isomorphically to a direct summand of the tangent space $T_{x_{n}} W^{s}$. 
For $J, M \in \mathbb{N}$, define $\Sigma_{J M}$ as the set of sequences $\left(a_{n}\right)_{n \in \mathbb{Z}} \in\{0, \ldots, J\}^{\mathbb{Z}}$ composed of blocks $12 \ldots J$ and of blocks of at least $M$ zeroes. $\Sigma_{J M}$ is a metric space with the metric $d\left(\left(a_{n}\right),\left(b_{n}\right)\right)=\sup _{n \in \mathbb{Z}} 2^{-|n|}\left|a_{n}-b_{n}\right|$. Define the shift operator $\sigma_{J M}: \Sigma_{J M} \longrightarrow \Sigma_{J M},\left(a_{n}\right) \mapsto\left(a_{n+1}\right)$.

If a $C^{1}$ map $\Pi$ has a transversally homoclinic orbit, all trajectories in a neighborhood of this orbit can be described by the symbol sequences from some space $\Sigma_{J M}$ ([SW]). The first result of this type for noninvertible maps in infinite dimension is due to Hale and Lin [HL1].

Our next aim is to establish a link between solutions of a delay equation homoclinic to a periodic solution and homoclinic orbits of associated Poincaré maps. We do this for general equations of type $(G)$ first, and then specialize to equations where the transversality criterion from [L-WW1] applies. Assume $G \in B C^{1}(C, \mathbb{R})$. Let $y: \mathbb{R} \longrightarrow \mathbb{R}$ be a periodic solution of eq. $(G)$ with minimal period $\eta>0$ and $H=h^{-1}(\{c\}) \subset C$ a hyperplane as in Theorem 2.4. Assume $y_{0} \in H, \quad h\left(\dot{y}_{\eta}\right) \neq 0$. Let $\mathcal{U} \subset C$ be an open bounded neighborhood of the set $\left\{y_{s}: s \in \mathbb{R}\right\}$. Then there exist bounded open neighborhoods $U \subset C$ of $y_{0}$ and $\mathcal{B} \subset B C^{1}(C, \mathbb{R})$ of $G$ and Poincaré maps $P_{F}: U \longrightarrow H(F \in \mathcal{B})$ with the properties described in Theorem 2.4 .

Define $U_{H}:=U \cap H$ and set $\pi:=\left.P_{G}\right|_{H}: U_{H} \longrightarrow H$. Set $H_{0}:=\operatorname{ker} h$. The hyperplane $H=y_{0}+H_{0}$ is, in general, not a vector space, but we can identify $H$ with the Banach space $H_{0}$ via the map $H_{0} \ni v \mapsto y_{0}+v$. Thus $\pi$ is a $C^{1}$ map on an open subset of a Banach space, and $D \pi(\psi) \in L_{c}\left(H_{0}, H_{0}\right)$ for $\psi \in U_{H}$. Obviously $\pi\left(y_{0}\right)=y_{0}$.

Lemma 2.6. Assume that the fixed point $y_{0}$ of $\pi$ is hyperbolic and unstable; let $W^{u}, W^{s} \subset U_{H}$ denote local unstable and stable manifolds of $\pi$ at $y_{0}$. Then there exists an open neighborhood $\Lambda \subset U_{H}$ of $y_{0}$ such that $\pi$ maps $\Lambda \cap W^{u}$ diffeomorphically onto $W^{u}$. Assume that $z: \mathbb{R} \longrightarrow \mathbb{R}$ is a solution of eq. $(G)$ different from $y$ and that $\left(t_{n}\right)_{n \in \mathbb{Z}}$ is a strictly increasing sequence in $\mathbb{R}$ with the subsequent properties:

(i) $z_{t_{n}}=\pi\left(z_{t_{n-1}}\right) \in W^{u} \cap \Lambda$ for $n<0$;

(ii) $z_{t_{0}}=\pi\left(z_{t_{-1}}\right) \in W^{u}$;

(iii) $z_{t_{1}} \in W^{s}, \quad \dot{z}_{t_{1}} \notin H_{0}$;

(iv) $z_{t_{n+1}}=\pi\left(z_{t_{n}}\right) \in W^{s}$ for $n \geq 1$.

Then there exist a bounded set $\mathcal{V} \subset C$, open bounded neighborhoods $U_{0} \subset U_{H}$ of $y_{0}$ in $H, U_{1}$ of $z_{t_{0}}$ in $H$ and $\mathcal{G}$ of $G$ in $B C^{1}(C, \mathbb{R})$, and $C^{1}$ Poincaré maps

$$
\Pi_{F}: U_{0} \cup U_{1} \longrightarrow H \subset C \quad(F \in \mathcal{G})
$$

with the following properties:

a) $\operatorname{dist}\left(U_{0}, U_{1}\right)>0$;

b) $\left\{z_{t_{n}}: n \neq 0\right\} \subset U_{0}$;

c) $\Pi_{G \mid U_{0}}=\pi_{\mid U_{0}}$

d) $\Pi_{G}\left(z_{t_{0}}\right)=z_{t_{1}}$;

e) if $\tilde{G} \in \mathcal{G}$ and $D \tilde{G}_{\mid \mathcal{V}}$ is uniformly continuous, then $D \Pi_{\tilde{G}}$ is uniformly continuous, and the map $\mathcal{G} \ni F \mapsto \Pi_{F} \in B C^{1}\left(U_{0} \cup U_{1}, H\right)$ is continuous at $\tilde{G}$;

f) with $\chi_{n}:=z_{t_{n}}(n \in \mathbb{Z})$, the sequence $\left(\chi_{n}\right)$ is a homoclinic orbit of $\Pi_{G}$. 
Proof. Choose a bounded open neighborhood $\mathcal{U}_{1}$ of $\left\{z_{t}: t \in \mathbb{R}\right\}$ in $C$. With $\mathcal{U}$ from the passage preceding the lemma, we set $\mathcal{V}:=\mathcal{U} \cup \mathcal{U}_{1}$. Note that, since eq. $(G)$ is autonomous, we have with $\varphi:=z_{t_{0}}$ that $x^{\varphi, G}(t)=z\left(t_{0}+t\right)$ for $t \geq 0$. The conditions $\dot{z}_{t_{1}} \notin H_{0}, z_{t_{1}} \in W^{s} \subset H$ imply the existence of bounded open neighborhoods $U_{1}^{\prime} \subset C$ of $z_{t_{0}}$ and $\mathcal{B}_{1}$ of $G$ in $B C^{1}(C, \mathbb{R})$ and of $C^{1}$ Poincaré maps $P_{F}^{(1)}: U_{1}^{\prime} \longrightarrow H \subset C$ as in Theorem 2.4. In particular, the analogous statement of Theorem 2.4 (iii) holds for $\tilde{G} \in \mathcal{B}_{1}$, if $D \tilde{G}_{\mid \mathcal{U}_{1}}$ is uniformly continuous.

With $U$ from the passage before the statement of the lemma, we have $z_{t_{n}} \in U$ for all $n \in \mathbb{Z}$. Since $W^{u}$ and $W^{s}$ are local unstable and stable manifolds of $\pi$, properties (i) and (iv) imply that $z_{t_{n}} \longrightarrow y_{0}$ for $n \longrightarrow \pm \infty$, so the set $Z:=$ $\left\{y_{0}\right\} \cup\left\{z_{t_{n}}: n \in \mathbb{Z} \backslash\{0\}\right\}$ is compact. Note that $z_{t_{0}} \notin Z$, since the $z_{t_{n}}(n \leq 0)$ are pairwise different and different from $y_{0}$, and since $W^{u} \cap W^{s}=\left\{y_{0}\right\}$. We can choose disjoint bounded open neighborhoods $U_{0}$ and $U_{1}$ of $Z$ resp. $z_{t_{0}}$ in $H$ such that $Z \subset U_{0} \subset U_{H}, U_{1} \subset U_{1}^{\prime} \cap U_{H}$, and $\operatorname{dist}\left(U_{0}, U_{1}\right)>0$. Assertions a) and b) are then true. Recall $\mathcal{B}$ from the passage preceding the lemma; set

$$
\mathcal{G}:=\mathcal{B}_{1} \cap \mathcal{B} \text {. }
$$

For $F \in \mathcal{G}$, we define $\Pi_{F}$ by

$$
\Pi_{F}(\psi):= \begin{cases}P_{F}^{(1)}(\psi), & \psi \in U_{1}, \\ P_{F}(\psi), & \psi \in U_{0} .\end{cases}
$$

The maps $\Pi_{F}$ are Poincaré maps. Assertion c) follows from $\left.P_{G}\right|_{U_{H}}=\pi$, and assertion d) follows from $z_{t_{0}} \in U_{1}$ and the construction of $P_{G}^{(1)}$.

Proof of assertion e): If $\tilde{G} \in \mathcal{G}$ and $D \tilde{G}_{\mid \mathcal{V}}$ is uniformly continuous, then obviously $D \tilde{G}_{\mid \mathcal{U}}$ and $D \tilde{G}_{\mid \mathcal{U}_{1}}$ are uniformly continuous. The construction of the maps $P_{G}^{(1)}$ and $P_{\tilde{G}}$ according to Theorem 2.4 implies that $D P_{\tilde{G}}^{(1)}$ is uniformly continuous on $U_{1}$ and that $D P_{\tilde{G}}$ is uniformly continuous on $U_{0}$. Since $\operatorname{dist}\left(U_{0}, U_{1}\right)>0$, it follows that $D \Pi_{\tilde{G}}$ is uniformly continuous. Further, the maps $\mathcal{B} \ni F \mapsto P_{F} \in B C^{1}(U, C)$ and $\mathcal{B}_{1} \ni F \mapsto P_{F}^{(1)} \in B C^{1}\left(U_{1}^{\prime}, C\right)$ are continuous at $\tilde{G}$. It follows trivially from $U_{0} \subset U, U_{1} \subset U_{1}^{\prime}$, and from the definition of the maps $\Pi_{F}$ that the map $\mathcal{G} \ni F \mapsto$ $\Pi_{F} \in B C^{1}\left(U_{0} \cup U_{1}, C\right)$ is continuous at $\tilde{G}$.

Proof of assertion $\mathrm{f}$ ): We have $\chi_{n}=z_{t_{n}} \longrightarrow y_{0}$ for $n \longrightarrow \pm \infty$. For $n<0$ and $n \geq 1$ we have $\chi_{n} \in U_{0}$, and the definition of $\Pi_{G}$ together with properties (i), (ii) and (iv) shows that

$$
\Pi_{G}\left(\chi_{n}\right)=P_{G}\left(\chi_{n}\right)=\pi\left(z_{t_{n}}\right)=z_{t_{n+1}}=\chi_{n+1} .
$$

For $n=0$, we have $\chi_{0}=z_{t_{0}} \in U_{1}$, and

$$
\Pi_{G}\left(\chi_{0}\right)=P_{G}^{(1)}\left(z_{t_{0}}\right)=z_{t_{1}}=\chi_{1} .
$$

It follows from $z \neq y$ that $\left(\chi_{n}\right)$ is nonconstant.

We obtain robust 'chaotic' trajectories of Poincaré maps if the homoclinic orbit $\left(\chi_{n}\right)$ from Lemma 2.6 has the transversality property. For arbitrary sets $A \subset B$ and maps $f: A \longrightarrow B$, we write $\operatorname{traj}(f, A)$ for the set of all trajectories of $f$ in $A$. We denote the maximal invariant subset of $f$ in $A$ by $\Omega(f, A)$; then

$$
x \in \Omega(f, A) \Longleftrightarrow \exists\left(x_{n}\right) \in \operatorname{traj}(f, A), \quad x_{0}=x .
$$


If $A$ is a bounded subset of a normed space with norm | |, we give $\operatorname{traj}(f, A)$ the topology induced by the metric $d\left(\left(x_{n}\right),\left(y_{n}\right)\right)=\sup _{n \in \mathbb{Z}} 2^{-|n|}\left|x_{n}-y_{n}\right| . f$ induces a shift operator $\hat{f}: \operatorname{traj}(f, A) \longrightarrow \operatorname{traj}(f, A), \quad\left(x_{n}\right) \mapsto\left(x_{n+1}\right)=\left(f\left(x_{n}\right)\right)$.

We do not repeat the definition of the term 'hyperbolic set' for noninvertible mappings, which is used in the theorem below. (See [SW], and, for equivalent properties, [L-WW2].) We just mention that the main difference in comparison to diffeomorphisms is that one gives up the invariance of the unstable directions under the derivative of the map.

Theorem 2.7. In the situation of Lemma 2.6, assume in addition that

a) the homoclinic orbit $\left(\chi_{n}\right)$ of $\Pi_{G}$ is transversal in the sense of Definition 2.5;

b) $D G_{\mid \mathcal{V}}$ is uniformly continuous.

Then there exists $\bar{\epsilon}_{0}>0$ such that for $\epsilon_{0} \in\left(0, \bar{\epsilon}_{0}\right]$ there exist $J, M \in \mathbb{N}$ open subsets $V_{0}, \ldots, V_{J}$ of $U_{0} \subset U_{1}$ and a neighborhood $\mathcal{F} \subset \mathcal{G}$ of $G$ in $B C^{1}(C, \mathbb{R})$ such that the following properties hold:

(i) $\operatorname{dist}\left(V_{i}, V_{j}\right)>0$ if $i, j \in\{0, \ldots, J\}, i \neq j$;

(ii) $\operatorname{diam}\left(V_{i}\right) \leq \epsilon_{0}, \quad i \in\{0, \ldots, J\}$.

(iii) Set $W:=V_{0} \cup \ldots \cup V_{J}$ and define the 'position' map $p: W \longrightarrow\{0, \ldots, J\}$, $p(x):=i$ if $x \in V_{i}$. For every $F \in \mathcal{F}$, the map

$$
\operatorname{traj}\left(\Pi_{F}, W\right) \longrightarrow\{0, \ldots, J\}, \quad\left(x_{n}\right) \mapsto\left(p\left(x_{n}\right)\right)
$$

maps into $\Sigma_{J M}$ and induces a homeomorphism $\hat{p}: \operatorname{traj}\left(\Pi_{F}, W\right) \longrightarrow \Sigma_{J M}$, with

$$
\hat{p} \circ \hat{\Pi}_{F}=\sigma_{J M} \circ \hat{p} ;
$$

(iv) for every $F \in \mathcal{F}, \quad \Omega\left(\Pi_{F}, W\right)$ is a hyperbolic set for $\Pi_{F}$.

Proof. Condition b) and part e) of Lemma 2.6 imply that $D \Pi_{G}$ is uniformly continuous. This fact, together with condition a), allows us to apply Theorems 6.4 , 6.5 and 6.6 from [L-W2]. We obtain $\bar{\epsilon}_{0}>0$ such that for $\epsilon_{0} \in\left(0, \bar{\epsilon}_{0}\right]$ there exist $J, M \in \mathbb{N}$ and open subsets $V_{0}, \ldots, V_{J}$ of $U_{0} \cup U_{1}$ with properties (i) and (ii); further, setting $W:=V_{0} \cup \ldots \cup V_{J}$, the position map $p$ induces a homeomorphism

$$
\tilde{p}: \operatorname{traj}\left(\Pi_{G}, W\right) \longrightarrow \Sigma_{J M}
$$

(see Theorem 6.4 from $[\mathrm{L}-\mathrm{W} 2]$ ). Let $\epsilon_{0} \in\left(0, \bar{\epsilon}_{0}\right]$. From Theorem 6.6 [L-W2], we obtain $\tilde{\epsilon}>0$ such that the $\tilde{\epsilon}$-neighborhood of $\Omega\left(\Pi_{G}, W\right)$ is contained in $W$, and with the following property: For every $\epsilon \in(0, \tilde{\epsilon}]$ there is a neighborhood $\Gamma_{\epsilon}$ of $\Pi_{G}$ in $B C^{1}\left(U_{0} \cup U_{1}, H\right)$ such that the following two statements hold for $\phi \in \Gamma_{\epsilon}$ :

1) For every trajectory $\left(x_{n}\right) \in \operatorname{traj}\left(\Pi_{G}, W\right)$ there exists a unique trajectory $\left(y_{n}\right) \in \operatorname{traj}(\phi, W)$ with $\left|y_{n}-x_{n}\right| \leq \epsilon(n \in \mathbb{Z})$, and the corresponding map $h$ : $\operatorname{traj}\left(\Pi_{G}, W\right) \longrightarrow \operatorname{traj}(\phi, W)$ is a homeomorphism.

2 ) The set $\Omega(\phi, W)$ is a hyperbolic set for $\phi$.

Choose now $\epsilon \in(0, \tilde{\epsilon}] \operatorname{such}$ that $\epsilon<\min _{i \neq j, i, j \in\{1, \ldots, n\}} \operatorname{dist}\left(V_{i}, V_{j}\right)$. Then, for $x \in \Omega\left(\Pi_{G}, W\right)$, one has $x \in V_{j}$ for some $j \in\{0, \ldots, J\}$, and for $y \in W$ with $|x-y| \leq \epsilon$, one also has $y \in V_{j}$, so that $p(x)=p(y)$.

For $\phi \in \Gamma_{\epsilon}$, the map $\tilde{p} \circ h^{-1}: \operatorname{traj}(\phi, W) \longrightarrow \Sigma_{J M}$ is a homeomorphism. If $\left(y_{n}\right)=h\left(\left(x_{n}\right)\right) \in \operatorname{traj}(\phi, W)$, we have $\left|x_{n}-y_{n}\right| \leq \epsilon(n \in \mathbb{Z})$, and

$$
\left(\tilde{p} \circ h^{-1}\right)\left(\left(y_{n}\right)_{n \in \mathbb{Z}}\right)=\tilde{p}\left(\left(x_{n}\right)_{n \in \mathbb{Z}}\right)=\left(p\left(x_{n}\right)_{n \in \mathbb{Z}}\right)=\left(p\left(y_{n}\right)\right)_{n \in \mathbb{Z}} .
$$


Thus $p$ induces the homeomorphism $\hat{p}:=\tilde{p} \circ h^{-1}$. For $\left(y_{n}\right) \in \operatorname{traj}(\phi, W)$, one has

$$
\begin{aligned}
(\hat{p} \circ \hat{\phi})\left(\left(y_{n}\right)\right) & =\hat{p}\left(\phi\left(y_{n}\right)\right)=\hat{p}\left(\left(y_{n+1}\right)\right)=\left(p\left(y_{n+1}\right)\right) \\
& =\sigma_{J M}\left[\left(p\left(y_{n}\right)\right)\right]=\left(\sigma_{J M} \circ \hat{p}\right)\left(\left(y_{n}\right)\right) .
\end{aligned}
$$

Condition b) and Part e) of Lemma 2.6 show that there exists a neighborhood $\mathcal{F} \subset \mathcal{G}$ of $G$ in $B C^{1}(C, \mathbb{R})$ such that for all $F \in \mathcal{F}$, one has $\Pi_{F} \in \Gamma_{\epsilon}$. It is now obvious that properties (iii) and (iv) hold for $F \in \mathcal{F}$.

In the above theorem, we had assumed the transversality property of the homoclinic orbit $\left(\chi_{n}\right)$. It has to be verified when we construct our example, and we employ the criterion from [L-WW1] that describes transversality in terms of oscillation properties. We now specialize to the case $G(\psi)=g(\psi(-1))$, that is, to delay equations of type $(g)$. If $x$ is a solution of $(g)$, the variational equation along $x$ is

$$
\dot{v}(t)=g^{\prime}(x(t-1)) v(t-1) .
$$

Lemma 2.8. Let $g \in B C^{1}(\mathbb{R}, \mathbb{R})$. Assume that $y: \mathbb{R} \longrightarrow \mathbb{R}$ is a periodic solution of $(g)$ with minimal period $\eta>0$. Assume further that the following conditions hold:

(i) y is slowly oscillating;

(ii) $g$ satisfies $(N F)$;

(iii) $g^{\prime}<0$ on the range $y(\mathbb{R})$ of $y$.

(iv) The spectrum of the monodromy operator $D_{2} \Phi^{g}\left(\eta, y_{0}\right)$ contains a point

$$
\lambda^{u} \in(1, \infty) \text {. }
$$

As in Lemma 2.6, let $\pi$ be a Poincaré map associated with $y$ and $\omega$. Then $y_{0}$ is a hyperbolic fixed point of $\pi$ with one-dimensional unstable space. If $W^{u}, W^{s}$ are local unstable and stable manifolds of $\pi$ at $y_{0}$ then $\operatorname{dim} W^{u}=1=\operatorname{codim} W^{s}$.

Proof. The assertions are proved in Section 6 of [L-WW1]. In that reference, it was assumed that $g^{\prime}<0$ on a neighborhood of $y(\mathbb{R})$, which follows from compactness of $y(\mathbb{R})$ and condition (iii). Further, the symmetry properties $y(t-2)=-y(t)(t \in \mathbb{R})$ and $g(\xi)=-g(-\xi)$ ) were assumed, but these properties are irrelevant for the proof of the above assertions; compare Remark 6.1 from [L-WW1].

Theorem 2.9. In the situation of Lemma 2.6 (with the homoclinic solution $z$ ), let the underlying equation be eq. $(g)$ with $g \in B C^{1}(C, \mathbb{R})$, and set $G(\psi):=$ $g(\psi(-1))(\psi \in C)$.

a) Then, with $\mathcal{V}$ from Lemma 2.6, $D G_{\mid \mathcal{V}}$ is uniformly continuous.

b) Assume in addition that $y$ and $g$ satisfy the conditions of Lemma 2.8. Set $\varphi:=z_{t_{0}} \in W^{u}$ and let $\omega \in H$ be a vector that spans the one-dimensional tangent space $T_{\varphi} W^{u}$. Consider the solution $w:\left[t_{0}-1, \infty\right) \longrightarrow \mathbb{R}$ of the variational equation $(g, z)$ with $w_{t_{0}}=\omega$. Assume that the following condition is satisfied:

(T) For all $(a, b) \in \mathbb{R}^{2} \backslash\{(0,0)\}$, the function $a \cdot \dot{z}+b \cdot w$ is eventually slowly oscillating.

Then the homoclinic orbit $\left(\chi_{n}\right)$ of $\Pi_{G}$ from Lemma 2.6,f) is transversal, and the conclusions of Theorem 2.7 hold.

c) Under the conditions of b), the domain $U_{0} \cup U_{1}$ of $\Pi_{G}$ can be chosen such that, for all trajectories of $\Pi_{G}$, the corresponding solutions of eq. $(g)$ are slowly oscillating. 
Proof. Ad a): Since $\mathcal{V}$ is bounded, the set $V:=\{\psi(-1): \psi \in V\} \subset \mathbb{R}$ is bounded, and $g^{\prime}$ is uniformly continuous on $V$; in particular, $g^{\prime}{ }_{\mid}$is uniformly continuous. For $\psi, \tilde{\psi} \in \mathcal{V}, \chi \in C$ we have

$$
|D G(\psi) \chi-D G(\tilde{\psi}) \chi| \leq\left|g^{\prime}(\psi(-1))-g^{\prime}(\tilde{\psi}(-1))\right||\chi|,
$$

and hence uniform continuity of $D G \mid \mathcal{V}$ follows from uniform continuity of $g^{\prime} \mid V^{\text {. }}$

Ad b): We have to show that the transversality property from Definition 2.5 holds. Set $n_{0}:=1$. From Lemma 2.6(i), we have

$$
\chi_{n}=z_{t_{n}} \in W^{u} \cap \Lambda \text { for } n \leq-1 \text { and } \chi_{n} \in W^{s} \text { for } n \geq 1 .
$$

Let now $m \leq-1, n \geq 1$. Let $\omega_{m}$ be a vector that spans $T_{\chi_{m}} W^{u}$. From the fact that $\pi$ induces a diffeomorphism on $W^{u} \cap \Lambda$ we see that there exists $\lambda \neq 0$ such that $D \pi^{|m|}\left(\chi_{m}\right) \omega_{m}=\lambda \omega$. It follows from assertions b) and c) of Lemma 2.6 that also

$$
D \Pi_{G}^{|m|}\left(\chi_{m}\right) \omega_{m}=\lambda \omega .
$$

For $j \geq 1$, let $p r_{j} \in L_{c}\left(H_{0}, H_{0}\right)$ denote the projection onto $H_{0}$ parallel to $\dot{z}_{t_{j}}$. Recall that the derivative $D \Pi^{G}$ is given by the derivative of the semiflow, $D_{2} \Phi^{G}$, followed by projection onto $H_{0}$ (see [DvGV-LW, p. 370, Proposition 3.2]), and that $D_{2} \Phi^{G}$ is given by solutions of the variational equation $(g, z)$. In formulas, we have

$$
D \Pi_{G}\left(z_{t_{0}}\right) \omega=\operatorname{pr}_{1}\left(D_{2} \Phi^{G}\left(t_{1}-t_{0}, z_{t_{0}}\right) \omega\right)=\operatorname{pr}_{1}\left(w_{t_{1}}\right) .
$$

There exists $\alpha \in \mathbb{R}$ with

$$
D \prod_{G}\left(z_{t_{0}}\right) \omega=w_{t_{1}}-\alpha \dot{z}_{t_{1}}
$$

Next, we have

$$
\begin{aligned}
& D \Pi_{G}^{n-1}\left(z_{t_{1}}\right)\left(w_{t_{1}}-\alpha \dot{z}_{t_{1}}\right)=\left[p r_{n} \circ D_{2} \Phi^{G}\left(t_{n}-t_{1}, z_{t_{1}}\right)\right]\left(w_{t_{1}}-\alpha \dot{z}_{t_{1}}\right) \\
= & p r_{n} D_{2} \Phi^{G}\left(t_{n}-t_{1}, z_{t_{1}}\right) w_{t_{1}}-\underbrace{\alpha \cdot p r_{n} \dot{z}_{t_{n}}}_{=0} \\
= & p r_{n} w_{t_{n}}
\end{aligned}
$$

and there exists $\beta \in \mathbb{R}$ with

$$
D \Pi_{G}^{n-1}\left(z_{t_{1}}\right)\left(w_{t_{1}}-\alpha \dot{z}_{t_{1}}\right)=w_{t_{n}}-\beta \dot{z}_{t_{n}} .
$$

Formulas (2.1), (2.2) and (2.3) together give

$$
\begin{aligned}
D \Pi_{G}^{n-m}\left(\chi_{m}\right) \omega_{m} & =D \Pi_{G}^{n-1}\left(z_{t_{1}}\right) D \Pi_{G}\left(z_{t_{0}}\right) D \Pi_{G}^{|m|}\left(\chi_{m}\right) \\
& =\lambda w_{t_{n}}-\lambda \beta \dot{z}_{t_{n}} .
\end{aligned}
$$

Set $\omega_{n}:=D \Pi_{G}^{n-m}\left(\chi_{m}\right) \omega_{m}$. Since codim $T_{\chi_{n}} W^{s}=1$, we have to show $\omega_{n} \notin T_{\chi_{n}} W^{s}$. Let $\zeta$ denote the solution of eq. $(g)$ with initial value $\zeta_{0}=\chi_{n}$, and let $\nu$ denote the solution of the variational eq. $(g, \zeta)$ with $\nu_{0}=\omega_{n}$. We then have

$$
\zeta(\cdot)=z\left(t_{n}+\cdot\right), \quad \nu(\cdot)=\lambda w\left(t_{n}+\cdot\right)-\lambda \beta \dot{z}\left(t_{n}+\cdot\right) .
$$

The transversality criterion from Theorem 8.2 of [L-WW1] shows that $\omega_{n} \notin T_{\chi_{n}} W^{s}$ if, for all $(a, b) \in \mathbb{R}^{2} \backslash\{(0,0)\}$, the function $a \dot{\zeta}+b \nu$ is eventually slowly oscillating. (Here we use that $g^{\prime}<0$ on $y(\mathbb{R})$.) In view of $(2.4)$, the latter condition is equivalent to the property that for all $(a, b) \in \mathbb{R}^{2} \backslash\{(0,0)\}$ the function $a \dot{z}+b w$ is eventually slowly oscillating, which is just condition $(\mathrm{T})$. Consequently, the homoclinic orbit 
$\left(\chi_{n}\right)$ is transversal. In view of assertion a), we see that the hypotheses of Theorem 2.7 are satisfied.

Ad c): Recall from Lemma 2.6 that $U_{0}$ and $U_{1}$ are neighborhoods of $y_{0}$ and $z_{t_{0}}$, respectively. Since $y$ is slowly oscillating, there exists $T>0$ with $y_{T}>0$ (see Proposition 4.6 from [L-WW1]). Hence there exists a neighborhood $U_{0,+}$ of $y_{0}$ in $C$ such that $x_{T}^{\psi}>0$ for all $\psi \in U_{0,+}$. As a first consequence, it follows from this property and from convergence of $z_{t}$ to the orbit of $y$ for $t \longrightarrow-\infty$ that there exist arbitrarily large $t>0$ with $z_{-t}>0$. Hence, since the set $S$ is invariant under the semiflow induced by the negative feedback equation $(g)$, the solution $z$ is slowly oscillating. Repeating the above argument for $z_{t_{0}}$ instead of $y_{0}$, we see that there exists a neighborhood $U_{1,+}$ of $z_{t_{0}}$ in $C$ and a $T_{1}>0$ such that $x_{T_{1}}^{\psi}>0$ for all $\psi \in U_{1,+}$. Assertion c) now follows if we choose $U_{0}$ and $U_{1}$ as subsets of $U_{0,+}$ and $U_{1,+}$, respectively. The proof is analogous to the proof of Corollary 5.4 from [L-WW2].

\section{Providing a starting VAlue and 'TARgets'}

An unstable slowly oscillating periodic solution. As in [L-WW2], we start with a function $g \in B C^{1}(\mathbb{R}, \mathbb{R})$ with the following properties:

$g^{\prime}<0, g^{\prime}$ is decreasing on an interval $[0, A](A>0)$. Further, $g(-x)=$ $-g(x)(x \in \mathbb{R})$, eq. $(g)$ has a slowly oscillating unstable periodic solution $y$ : $\mathbb{R} \longrightarrow \mathbb{R}$ with $y(\mathbb{R}) \subset[-A, A] . y$ has the symmetry

$$
y(t-2)=-y(t) \quad(t \in \mathbb{R})
$$

and is called a Kaplan-Yorke solution, since such symmetric solutions were first obtained by Kaplan and Yorke [KY]. The phase of $y$ is chosen such that

$$
y(-1)=0, \quad y>0 \text { on }(-1,1) .
$$

In the notation [L-WW2], we have $(y, g) \in Y G$. On the space $C=C^{0}([-1,0], \mathbb{R})$, we write || for ||$_{C^{0}}$, unless the more explicit notation seems favourable. For a normed space $(E,||)$ and $r>0$, we use the notation $E(r)$ for the open ball with radius $r$ in $E$.

Set $H:=\{\psi \in C: \quad \psi(-1)=0\}$ and let $\mathcal{O}:=\left\{y_{t}: t \in \mathbb{R}\right\}$ denote the orbit of $y$ in $C$. We have $y_{0}=y_{4} \in H, \dot{y}_{4} \notin H$. Let us recall some facts from [L-WW2]: There exists an open neighborhood $U_{P}$ of $y_{0}$ in $H$ and a Poincaré map $P: U_{P} \longrightarrow H \subset C$. We have $P \in B C^{1}\left(U_{P}, H\right), \quad P\left(y_{0}\right)=y_{0}$, and $D P\left(y_{0}\right)$ is hyperbolic with unstable space $U \subset H$, stable space $Z \subset H$, and $\operatorname{dim} U=1$.

There exists a norm \|\| on $H$ equivalent to ||$_{C^{0}}$ such that $D P\left(y_{0}\right)$ is expanding on $U$ and contracting on $Z$ w.r. to \|\| , and such that $\|v+w\|=\max \{\|v\|,\|w\|\}$ for $v \in Z, w \in U$. For $\delta>0$ and a subspace $W \subset H$, we set

$$
W_{\|\|}(\delta):=\{\psi \in W:\|\psi\|<\delta\} \text { and } W(\delta):=\left\{\psi \in W:|\psi|_{C^{0}}<\delta\right\} .
$$

Set

$$
N_{\delta}:=\left\{\psi \in H:\left\|\psi-y_{0}\right\|<\delta\right\}=y_{0}+Z_{\|\|}(\delta)+U_{\|\|}(\delta) .
$$

For $\psi \in C$, let $x^{\psi}:[-1, \infty) \longrightarrow \mathbb{R}$ denote the solution of eq. $(g)$ with $x_{0}=\psi$.

We can choose $\bar{\delta} \in(0,1 / 4]$ with the following properties: For $\delta \in(0, \bar{\delta}]$, the local unstable and stable sets $W^{u}\left(y_{0}, P, N_{\delta}\right), W^{s}\left(y_{0}, P, N_{\delta}\right)$ coincide with the graphs

$$
y_{0}+\left\{\psi+w^{u}(\psi): \psi \in U_{\|\|}(\delta)\right\}, \quad y_{0}+\left\{\psi+w^{s}(\psi): \psi \in Z_{\|\|}(\delta)\right\}
$$


of $C^{1}$-maps $w^{u}: U_{\|\|}(\delta) \longrightarrow Z_{\|||}(\delta) \subset Z, \quad w^{s}: Z_{\|\| \mid}(\delta) \longrightarrow U_{\|\|}(\delta) \subset U$, respectively. One has $w^{u}(0)=0, w^{s}(0)=0, D w^{u}(0)=0, D w^{s}(0)=0$.

For $f: \mathbb{R} \longrightarrow \mathbb{R}$ and $M \subset \mathbb{R}$, by a modification of $f$ on $\mathbb{R} \backslash M$ we mean a function $\tilde{f}: \mathbb{R} \longrightarrow \mathbb{R}$ with $\tilde{f}(x)=f(x)$ for $x \in M$. We will construct modifications of $g$ outside the range of $y$. The aim is that solutions of the modified equation which start in some unstable set $W^{u}\left(y_{0}, P, N_{\delta}\right)$ have their segments at a time approximately equal to 3 in a small neighborhood of $y_{0}$.

We first prove some preparatory statements that hold for all sufficiently small $\delta>0$, where $\delta$ is essentially a measure for the distance of the starting value in $W^{u}\left(y_{0}, P, N_{\delta}\right)$ to $y_{0}$. Later on, we pick an appropriate fixed $\delta>0$. This is an important technical detail of our construction.

Proposition 3.1. There exist $k_{1} \in(0,1], k_{2}>0, k_{3} \in(0,1]$ such that the subsequent properties hold for all $\delta \in(0, \bar{\delta}]$.

a) $\psi \in N_{k_{1} \delta} \Longrightarrow\left|\psi-y_{0}\right|<\delta$;

b) $\forall \psi \in W^{s}\left(y_{0}, P, N_{k_{1} \delta}\right) \forall t \geq 0: \quad x_{t}^{\psi} \in \mathcal{O}+C(\delta)$;

c) $\psi \in H,\left|\psi-y_{0}\right| \leq k_{3} \delta \Longrightarrow x^{\psi}(1-\delta) \geq k_{2} \delta$.

Proof. There exist $K>0$ and $\lambda>0$ with the property

$$
\forall \psi \in W^{s}\left(y_{0}, P, N_{\bar{\delta}}\right): \quad \forall t \geq 0: \quad \operatorname{dist}\left(x_{t}^{\psi}, \mathcal{O}\right) \leq K \exp (-\lambda t)\left|\psi-y_{0}\right| .
$$

(Here, 'dist' refers to ||$=||_{C^{0}}$.) Choose $T>1$ such that $K \exp (-\lambda T)<1$. There exists $c>0$ such that

$$
\forall \psi, \chi \in C \forall t \in[0, T]: \quad\left|x^{\psi}(t)-x^{\chi}(t)\right| \leq c|\chi-\psi|
$$

There exist constants $c_{1}, c_{2}>0$ such that the estimates \|\|$\leq c_{1}||$ and ||$\leq c_{2}\|\|$ hold. Set $k_{1}:=\frac{1}{2\left(c c_{2}+c_{2}+1\right)}$. For $\psi \in N_{k_{1} \delta}$, one has $\left|\psi-y_{0}\right| \leq c_{2} k_{1} \delta<\delta$, hence property a) holds.

If $\psi \in W^{s}\left(y_{0}, P, N_{k_{1} \delta}\right)$ then $\left|x^{\psi}(t)-y(t)\right| \leq c c_{2} k_{1} \delta$ for $t \in[0, T]$, so $\operatorname{dist}\left(x_{t}^{\psi}, \mathcal{O}\right) \leq$ $\max \left\{c_{2} k_{1} \delta ; c c_{2} k_{1} \delta\right\}<\delta$ for $t \in[0, T]$. For $t \geq T$, we have

$$
\operatorname{dist}\left(x_{t}^{\psi}, \mathcal{O}\right) \leq K \exp (-\lambda T)\left|\psi-y_{0}\right| \leq 1 \cdot c_{2} k_{1} \delta<\delta
$$

and property $\mathrm{b})$ is proved.

Note that $\left|x^{\psi}(1)-y(1)\right|=\left|x^{\psi}(1)\right| \leq c\left|\psi-y_{0}\right|$ for $\psi \in C$. For $t \in(1 / 2,1], \psi \in C$ with $\left|\psi-y_{0}\right| \leq 1$, one has

$$
\left|\dot{x}^{\psi}(t)-\dot{y}(t)\right|=\left|g\left(x^{\psi}(t-1)\right)-g(y(t-1))\right| \leq|g|_{C^{1}}\left|\psi-y_{0}\right| .
$$

Set

$$
\mu:=\min \{|g(y(s))|: s \in[-1 / 2,0]\}, \quad k_{3}:=\min \left\{\frac{\mu}{2\left(c+|g|_{C^{1}}\right)}, 1\right\} .
$$


On $[1 / 2,1]$ we have $|\dot{y}(t)|=|g(y(t-1))| \geq \mu>0$, which implies $y(1-\delta) \geq \mu \cdot \delta$ for $\delta \in[0,1 / 2]$. For $\delta \in(0, \bar{\delta}]$ and $\psi \in H$ with $\left|\psi-y_{0}\right| \leq k_{3} \delta$, one has

$$
\begin{aligned}
x^{\psi}(1-\delta) & =x^{\psi}(1)+\int_{1}^{1-\delta} \dot{x}^{\psi}(s) d s \\
& \geq \underbrace{y(1)}_{=0}+\int_{1}^{1-\delta} \dot{y}(s) d s-\left|x^{\psi}(1)-y(1)\right|-\int_{1}^{1-\delta}\left|\dot{y}(s)-\dot{x}^{\psi}(s)\right| d s \\
& \geq y(1-\delta)-c k_{3} \delta-\delta|g|_{C^{1}} k_{3} \delta \\
& \geq\left(\mu-\left(c+|g|_{C^{1}}\right) k_{3}\right) \delta \geq \frac{\mu}{2} \delta .
\end{aligned}
$$

Property c) now follows if we put $k_{2}:=\mu / 2$.

It was shown in Section 3 of [L-WW2] that there exists an eigenvector $\chi^{u} \in H$ of $D P\left(y_{0}\right)$ with $U=\mathbb{R} \cdot \chi^{u}, \chi^{u}(0)>0, \dot{\chi}^{u}>0$ on $[-1,0)$. We can choose $\chi^{u}$ such that $\left\|\chi^{u}\right\|=1$. Let $\pi^{u}, \pi^{s}$ denote the projections onto $U$, respectively $Z$, according to $H=U \oplus Z$. Recall the set $S \subset C$ of segments with at most one sign change. It is known that $S \cap Z=\emptyset$ (see, e.g., Corollary 5.4 from [L-WW1]). Since $y_{0} \in S$, we have $\pi^{u} y_{0} \neq 0$, and so there exists a unique real number $\eta_{0}>0$ with $\pi^{u} y_{0}=\eta_{0} \cdot \chi^{u}$. Define $i: \mathbb{R} \longrightarrow U, r \mapsto r \cdot \eta_{0} \cdot \chi^{u}$. The map $i$ is an isomorphism, and $i^{-1}\left(\pi^{u}\left(y_{0}\right)\right)=1$. Set

$$
\mathcal{H}: N_{\bar{\delta}} \longrightarrow \mathbb{R}, \quad \mathcal{H}(\psi):=i^{-1}\left[\pi^{u}\left(\psi-y_{0}\right)-w^{s}\left(\pi^{s}\left(\psi-y_{0}\right)\right)\right] .
$$

Then, for all $\delta \in(0, \bar{\delta}]$ and $\psi \in N_{\delta}$, we have the equivalence

$$
\psi \in W^{s}\left(y_{0}, P, N_{\delta}\right) \Longleftrightarrow \mathcal{H}(\psi)=0 .
$$

We provide a Lipschitz estimate for $\mathcal{H}$ : For $\chi \in U, \chi=\lambda \cdot \chi^{u}$, we have $\left\|\chi^{u}\right\|=\lambda$ and

$$
\left|i^{-1} \chi\right|=\left|i^{-1}\left(\frac{\lambda}{\eta_{0}} \cdot \eta_{0} \chi^{u}\right)\right|=\frac{|\lambda|}{\eta_{0}}=\frac{1}{\eta_{0}}\|\chi\| .
$$

Since $w^{s}$ is $C^{1}$ and $D w^{s}(0)=0$, there exists $\tilde{\delta} \in(0, \bar{\delta}]$ such that $w^{s}$ has Lipschitz constant 1 on $Z_{\|\| \|}(\tilde{\delta})$. Since the operator norms $\left\|\pi^{u}\right\|$ and $\left\|\pi^{s}\right\|$ are equal to one, we obtain for $\chi, \psi \in N_{\tilde{\delta}}$ :

$$
|\mathcal{H}(\psi)-\mathcal{H}(\chi)| \leq \frac{1}{\eta_{0}}\left[\left\|\pi^{u}\right\| \cdot\left\|\psi-y_{0}\right\|+\left\|\pi^{s}\right\| \cdot\left\|\psi-y_{0}\right\|\right]=\frac{2}{\eta_{0}}\left\|\psi-y_{0}\right\| .
$$

We now provide 'target' segments. Later, we want to steer the phase curves of solutions starting in some unstable manifold of $y_{0}$ into a neighborhood of these targets.

Proposition 3.2. Set $\varphi_{\lambda}:=(1+\lambda) y_{0}$ for $\lambda \in \mathbb{R}$. There exists $\lambda_{0} \in(0,1)$ such that, for $\lambda \in\left[-\lambda_{0}, \lambda_{0}\right]$, one has

$$
\mathcal{H}\left(\varphi_{\lambda}\right) \geq \frac{1}{2} \lambda \text { if } \lambda>0, \quad \mathcal{H}\left(\varphi_{\lambda}\right) \leq \frac{1}{2} \lambda \text { if } \lambda<0 .
$$

Proof. Since $w^{s}(0)=0, D w^{s}(0)=0$, there exists $r \in(0,1)$ such that for $\chi \in Z$ with $\|\chi\| \leq r$,

$$
\left\|w^{s}(\chi)\right\| \leq \frac{\eta_{0}}{2\left(\left\|\pi^{s} y_{0}\right\|+1\right)}\|\chi\|
$$


Set $\lambda_{0}:=\frac{r}{\left(\left\|\pi^{s} y_{0}\right\|+1\right)}$. For $\lambda \in\left[-\lambda_{0}, \lambda_{0}\right]$, we have

$$
\left\|\pi^{s}\left(\varphi_{\lambda}-y_{0}\right)\right\|=|\lambda| \cdot\left\|\pi^{s} y_{0}\right\| \leq \lambda_{0}\left\|\pi^{s} y_{0}\right\| \leq r
$$

and

$$
w^{s}\left(\pi^{s}\left(\varphi_{\lambda}-y_{0}\right)\right)\left\|\leq \frac{\eta_{0}}{2\left(\left\|\pi^{s} y_{0}\right\|+1\right)}|\lambda| \cdot\right\| \pi^{s} y_{0} \| \leq \frac{\eta_{0}}{2}|\lambda| .
$$

It follows that, for $\lambda \in\left[-\lambda_{0}, \lambda_{0}\right]$, one has

$$
\left|i^{-1}\left(w^{s}\left(\varphi_{\lambda}-y_{0}\right)\right)\right| \leq \frac{1}{\eta_{0}} \frac{\eta_{0}}{2}|\lambda|=\frac{|\lambda|}{2} .
$$

We obtain

$$
\begin{aligned}
\mathcal{H}\left(\varphi_{\lambda}\right) & =i^{-1}\left[\pi^{u}\left(\lambda y_{0}\right)-w^{s}\left(\pi^{s}\left(\varphi_{\lambda}-y_{0}\right)\right)\right] \\
& =\lambda \underbrace{i^{-1}\left(\pi^{u} y_{0}\right)}_{=1}-i^{-1} w^{s}\left(\pi^{s}\left(\varphi_{\lambda}-y_{0}\right)\right) \\
& \in\left[\lambda-\frac{|\lambda|}{2}, \lambda+\frac{|\lambda|}{2}\right],
\end{aligned}
$$

and the assertion follows.

Proposition 3.3. There exist $\delta^{\prime} \in(0, \tilde{\delta}]$ and constants $M_{2}>0, k_{4} \in(0,1], d_{2}>0$ such that for all $\iota \in\left(0, \delta^{\prime}\right]$ there exist functions $\psi_{+}, \psi_{-} \in N_{\iota}$ with the following properties:

(i) $\psi_{+}, \psi_{-}$are $C^{2}, \quad \dot{\psi}_{ \pm}>0$ on $[-1,0), \dot{\psi}_{ \pm}(0)=0$, and $\ddot{\psi}_{ \pm}<0$ on $[-1,0]$, and $\ddot{\psi}_{ \pm}(t) \leq-d_{2}<0$ for $t \in[-1 / 2,0]$.

(ii) $\left|\psi_{ \pm}\right|_{C^{2}} \leq M_{2}$.

(iii) For all $\psi \in \psi_{+}+\overline{H\left(k_{4} \iota\right)}$ and for all $\chi \in \psi_{-}+\overline{H\left(k_{4} \iota\right)}$, one has $\psi, \chi \in N_{\iota}$, and

$$
\mathcal{H}(\psi)>0>\mathcal{H}(\chi)
$$

Proof. Choose $\lambda_{0}$ as in Proposition 3.2, and choose $\delta^{\prime} \in(0, \tilde{\delta}]$ such that $\delta^{\prime}<$ $2\left\|y_{0}\right\| \cdot \lambda_{0}$. Set $M_{2}:=\left(\lambda_{0}+1\right)\left|y_{0}\right|_{C^{2}}+2$ and $k_{4}:=\min \left\{\frac{\eta_{0}}{32\left(\left\|y_{0}\right\|+1\right)}, \frac{1}{5}\right\}$. It follows from eq. ( $g$ ) that there exists $D_{2}>0$ such that $y_{0}^{\prime \prime}(t) \leq-D_{2}<0$ for $t \in[-1 / 2,0]$. Set $d_{2}:=\left(1-\lambda_{0}\right) D_{2}$. Let now $\iota \in\left(0, \delta^{\prime}\right]$ be given. Set $\lambda:=\frac{\iota}{2\left(\left\|y_{0}\right\|+1\right)}$; then $\lambda \leq \lambda_{0}$. With $\varphi_{\lambda}$ as in Proposition 3.2, we have

$$
\left\|\varphi_{\lambda}-y_{0}\right\|=\left\|\lambda y_{0}\right\| \leq \frac{\iota}{2}<\tilde{\delta}, \text { and } \mathcal{H}\left(\varphi_{\lambda}\right) \geq \frac{\lambda}{2}
$$

If $\psi \in \varphi_{\lambda}+H_{\|\|}\left(2 k_{4} \iota\right)$ then

$$
\left\|\psi-y_{0}\right\| \leq 2 k_{4} \iota+\left\|\varphi_{\lambda}-y_{0}\right\|=\left(2 k_{4}+1 / 2\right) \iota<\iota \leq \tilde{\delta}
$$

so we have $\psi, \varphi_{\lambda} \in N_{\tilde{\delta}}$. Using the Lipschitz estimate for $\mathcal{H}$, we obtain for all $\psi \in \varphi_{\lambda}+H_{\|\|}\left(2 k_{4} \iota\right)$ :

$$
\mathcal{H}(\psi) \geq \mathcal{H}\left(\varphi_{\lambda}\right)-\frac{2}{\eta_{0}} \cdot 2 k_{4} \iota \geq \frac{\lambda}{2}-\frac{\iota}{8\left(\left\|y_{0}\right\|+1\right)}=\frac{\iota}{8\left(\left\|y_{0}\right\|+1\right)}
$$

Set $p(x):=(x-1)(x+1)$ for $x \in[-1,0]$. Then

$$
p^{\prime}(x)=2 x<0 \text { for } x \in[-1,0), \quad p^{\prime}(0)=0, \quad p^{\prime \prime}(x)=2 \text { for } x \in[-1,0],
$$

and $|p|_{C^{2}}=\max \left\{|p|_{C^{0}},\left|p^{\prime}\right|_{C^{0}},\left|p^{\prime \prime}\right|_{C^{0}}\right\} \leq \max \{1,2,2\}=2$. 
Consider now the functions $\varphi_{\lambda, \epsilon}:=\varphi_{\lambda}-\epsilon \cdot p$ for $\epsilon \in(0,1)$. These functions are $C^{2}$ and have the following properties:

$$
\begin{gathered}
\varphi_{\lambda, \epsilon}^{\prime}=\underbrace{(1+\lambda) y_{0}^{\prime}}_{\geq 0}-\epsilon p^{\prime}>0 \text { on }[-1,0), \quad \varphi_{\lambda, \epsilon}^{\prime}(0)=0, \\
\varphi_{\lambda, \epsilon}^{\prime \prime}=\underbrace{(1+\lambda) y_{0}^{\prime \prime}}_{\leq 0}-2 \epsilon<0 \text { on }[-1,0],
\end{gathered}
$$

and

$$
\varphi_{\lambda, \epsilon}^{\prime \prime}(x) \leq \varphi_{\lambda}^{\prime \prime}(x)=(1+\lambda) y_{0}^{\prime \prime}(x) \leq-\left(1-\lambda_{0}\right) D_{2}=-d_{2}<0
$$

for $x \in[-1 / 2,0]$. Further, we have

$$
\left|\varphi_{\lambda, \epsilon}\right|_{C^{2}} \leq\left|\varphi_{\lambda}\right|_{C^{2}}+\epsilon|p|_{C^{2}} \leq\left(\lambda_{0}+1\right)\left|y_{0}\right|_{C^{2}}+2=M_{2} .
$$

Choose $\epsilon \in(0,1]$ such that $\left\|\varphi_{\lambda, \epsilon}-\varphi_{\lambda}\right\|<k_{4} \iota$ and set $\psi_{+}:=\varphi_{\lambda, \epsilon}$. Then $\psi_{+}$ has the properties asserted in (i) and (ii). Let now $\psi \in \psi_{+}+H_{\|\|}\left(k_{4} \iota\right)$; then $\psi \in \varphi_{\lambda}+H_{\|\|}\left(2 k_{4} \iota\right)$, so $(3.3)$ shows that $\mathcal{H}(\psi)>0$. Further, since $\left\|\varphi_{\lambda}-y_{0}\right\|=$ $\left\|\lambda y_{0}\right\| \leq \iota / 2$, and $\iota / 2+2 k_{4} \iota<(1 / 2+2 / 5) \iota<\iota$, we have $\psi_{ \pm} \in N_{\iota}$. Consequently, $\psi_{+}$also has the properties stated in item (iii). The construction of $\psi_{-}$satisfying conditions (i)-(iii) is analogous, setting $\lambda:=-\frac{\iota}{2\left(\left\|y_{0}\right\|+1\right)}$.

The next proposition is the main step towards the choice of an initial segment through which there is a backward solution of eq. $(g)$, the phase curve of which converges to the periodic orbit $\mathcal{O}$. Later, we will find a modified equation such that the backward phase curve is preserved and the forward phase curve also converges to $\mathcal{O}$. In the proof of Proposition 3.4 below, we refer to the proof of Proposition 3.1 from [L-WW2], which is partially analogous.

Proposition 3.4. For every $\hat{\delta} \in(0, \bar{\delta}]$, there exist $\varphi \in W^{u}\left(y_{0}, P, N_{\bar{\delta}}\right)$ and $\delta_{1} \in$ $(0, A-y(0))$ with the subsequent properties. (With $k_{3}$ from Proposition 3.1, set $\delta:=\frac{2\left|\varphi-y_{0}\right|}{k_{3}}$, so that $\left|\varphi-y_{0}\right|=k_{3} \delta / 2$.)

a) $\delta \leq \hat{\delta}, \varphi(0)<A, \varphi(0)-y(0)>\delta_{1} \geq k_{3} \delta / 8, \delta_{1} \leq \bar{\delta}$. Further,

$$
\varphi(-1 / 2) \geq y(-1 / 2) / 2 .
$$

b) There exists a solution $X: \mathbb{R} \longrightarrow \mathbb{R}$ of eq. (g) with $X_{0}=\varphi$.

c) $\dot{X}>0$ on $[-1,0), \quad \dot{X}<0$ on $(0,1], \quad X(-1)=0$.

d) There exist $\theta_{-}, \theta_{+} \in(0,1 / 4)$ such that

$$
\begin{aligned}
X(t) & >y(0)+\delta_{1} \text { for } t \in\left(-\theta_{-}, \theta_{+}\right) ; \\
X\left(-\theta_{-}\right) & =X\left(\theta_{+}\right)=y(0)+\delta_{1} ; \\
-y(0)-1 / 2 & <X(t)<y(0)+\delta_{1} \text { for } t \in\left(-\infty,-\theta_{-}\right) ; \\
-y(0)-1 / 2 & \leq X(t) \text { for } t \in(-\infty, 1] .
\end{aligned}
$$

e) $\theta_{-} \leq \delta, \quad \theta_{+} \leq \delta$

f) $|g(y(t))-g(X(t))| \leq y(0) / 4$ for $t \in[0,1]$.

g) The tangent space $T_{\varphi} W^{u}\left(y_{0}, P, N_{\bar{\delta}}\right)$ is spanned by a segment $\omega \in H$ with $\omega(0)>0$.

h) The solution $w:[-1, \infty) \longrightarrow \mathbb{R}$ of the variational equation $(g, X)$ with $w_{0}=\omega$ satisfies $w(t)>0$ for $t \in\left[-\theta_{-}, \theta_{+}\right]$. 
Proof. 1. Assume that properties b), c) and d) hold for some $\varphi$ with $\varphi(0)>y(0)$ and some $\delta_{1} \in(0, \varphi(0)-y(0))$, and for corresponding numbers $\theta_{-}, \theta_{+}$. Then the analogous properties hold for every $\tilde{\delta}_{1} \in\left[\delta_{1}, \varphi(0)-y(0)\right)$, with the corresponding numbers $\tilde{\theta}_{-}, \tilde{\theta}_{+}$. These $\tilde{\theta}_{ \pm}$are uniquely determined, in view of c), and we have $\lim _{\tilde{\delta}_{1} \longrightarrow \varphi(0)-y(0)} \tilde{\theta}_{ \pm}=0$.

2. It was shown in Proposition 3.1 of [L-WW2] that there exists $\varphi \in W^{u}\left(y_{0}, P, N_{\bar{\delta}}\right)$ such that properties b),c),d), f) and g) hold. (Concerning property f), note that the number $L$ from part b) of that proposition is a Lipschitz constant for $g$.) It is clear from the construction that these properties can be achieved with $\varphi$ arbitrarily close to $y_{0}$.

3. All $\varphi \in W^{u}\left(y_{0}, P, N_{\bar{\delta}}\right)$ with $\varphi(0)>y(0)$ and sufficiently close to $y_{0}$ are of the form $\varphi=\varphi_{r}=y_{0}+r \cdot \chi^{u}+w^{u}\left(r \cdot \chi^{u}\right)$ for some uniquely determined $r>0$. If $r$ is so small that

$$
\left|w^{u}\left(r \chi^{u}\right)\right| \leq \frac{1}{3} r\left|\chi^{u}\right|,
$$

then, using $\left|\chi^{u}\right|=\chi^{u}(0)$, we obtain

$$
\begin{aligned}
\left|\varphi_{r}(0)-y(0)\right| & \geq r \chi^{u}(0)-\frac{1}{3} r \chi^{u}(0)=\frac{2}{3} r \cdot \chi^{u}(0) \\
& =\frac{1}{2} \cdot \frac{4}{3} r\left|\chi^{u}\right| \\
& \geq \frac{1}{2}\left|r \chi^{u}+w^{u}\left(r \cdot \chi^{u}\right)\right| \\
& =\frac{1}{2}\left|\varphi_{r}-y_{0}\right| .
\end{aligned}
$$

4. Let now $\hat{\delta} \in(0, \bar{\delta}]$. In view of steps 2 and 3 , we can choose $r>0$ so small that $\varphi:=\varphi_{r}$ satisfies

$$
\varphi(0)<A,\left|\varphi-y_{0}\right| \leq \frac{k_{3} \hat{\delta}}{2}, \varphi(0)-y(0) \leq \bar{\delta}, \varphi(-1 / 2) \geq y(-1 / 2) / 2,
$$

and that properties b), c), d), f) and g) hold with some $\tilde{\delta}_{1} \in(0, \varphi(0)-y(0))$ and corresponding numbers $\tilde{\theta}_{ \pm}$. Then, setting $\delta:=2\left|\varphi-y_{0}\right| / k_{3}$, we have $\delta \leq \hat{\delta}$. Using step 1), we can choose $\delta_{1} \in\left[\tilde{\delta}_{1}, \varphi(0)-y(0)\right)$ so close to $\varphi(0)-y(0)$ that properties b), c), d), f) and g) also hold with $\delta_{1}$ and the corresponding numbers $\theta_{ \pm}$instead of $\tilde{\delta}_{1}$ and $\tilde{\theta}_{ \pm}$, and that, in addition, the following conditions are satisfied:

(i) $\theta_{-}, \theta_{+} \leq \delta$, so that property e) holds;

(ii) property h) holds (here we use the continuity of $w$ );

(iii) $\delta_{1} \geq \frac{\varphi(0)-y(0)}{2}$.

It remains now to check that a) holds. In view of (3.6) and the choice of $\delta_{1}$ and $\delta$, we only have to prove the inequalities $\delta_{1} \leq \bar{\delta}$ and $\delta_{1} \geq k_{3} \delta / 8$. First, $\delta_{1} \leq \varphi(0)-y(0) \leq \bar{\delta}$. Second, from (ii) above and from (3.5), we get

$$
\delta_{1} \geq \frac{\varphi(0)-y(0)}{2} \geq \frac{1}{4}\left|\varphi-y_{0}\right|=k_{3} \delta / 8 .
$$


We are now ready to choose an initial value and targets. Set

$$
\gamma_{2}:=\left|g^{\prime}(0)\right|\left|g\left(y_{0}(-1 / 2) / 2\right)\right|>0 \text { and } c_{g}:=\sqrt{\frac{|g|_{C^{0}}}{\gamma_{2}}} .
$$

Recall that $\delta^{\prime} \leq \tilde{\delta} \leq \bar{\delta} \leq 1 / 4$. Choose $\hat{\delta} \in\left(0, \delta^{\prime}\right]$ such that the following inequalities hold for all $\delta \in(0, \hat{\delta}]$ :

(i) $y(2-\delta)-y(1+\delta) \leq-y(0) / 2$;

(ii) $2 \delta|g|_{C^{0}} \leq y(0) / 8$;

(iii) $12 \delta|g|_{C^{1}} \leq 1 / 2$;

(iv) $2 c_{g} \sqrt{\delta} \leq 1 / 2-\delta, \quad \delta \leq c_{g} \cdot \sqrt{\delta}$;

(v) $10 M_{2} c_{g} \delta^{3 / 2} \leq \frac{k_{4} k_{1} k_{3} \delta}{64}$;

(vi) $\frac{9 M_{2}}{2} \delta^{2} \leq \frac{k_{4} k_{1} k_{3} \delta}{32}$.

We now apply Proposition 3.4 with this number $\hat{\delta}$. From now on, let $\varphi, \delta \in$ $(0, \hat{\delta}], X, \theta_{ \pm}, \delta_{1} \in\left[k_{3} \delta / 4, k_{3} \delta / 2\right]$, and $\omega$ be as in Proposition 3.4. We set

$$
\iota:=k_{1} \delta_{1} \text {. }
$$

Then $\iota \leq \delta_{1}<\delta \leq \hat{\delta} \leq \delta^{\prime}$, since $k_{1}, k_{3} \leq 1$. Applying Proposition 3.3 with this number $\iota$, we obtain $\psi_{+}, \psi_{-} \in N_{\iota}$ with the properties listed in items (i)-(iii) of Proposition 3.3. Set

$$
\psi_{s}:=(1-s) \psi_{-}+s \psi_{+} \in N_{\iota} \text { for } s \in[0,1]
$$

these functions $\psi_{s}$ will be the targets.

\section{Successive modifications of $g$}

We obtain our example by successive deformations of the original nonlinearity $g$, which satisfies $g^{\prime}<0$. The modifications are such that the unstable periodic solution $y$ of eq. $(g)$, together with local unstable and stable manifolds, is preserved. The initial value $\varphi$ from the unstable manifold leads to a homoclinic solution for the finally obtained equation.

The methods are partially similar to the ones used in the previous example from [L-WW2]. However, obtaining the simpler shapes of both the nonlinearity and the homoclinic solution requires finer techniques for estimates on solution behavior, compared to [L-WW2]. In addition, the necessity to avoid zeroes of the derivative of the nonlinearity (except for one) leads to some technical complications. We have already encountered one of these technicalities - the addition of the term $-\epsilon p$ to $\varphi_{\lambda}$ in the proof of Proposition 3.3, which had the purpose to achieve $\ddot{\psi}_{ \pm}<0$ on the whole interval $[-1,0]$.

If $h: \mathbb{R} \longrightarrow \mathbb{R}$ is a continuous modification of $g$ on $\mathbb{R} \backslash\left[-y(0)-1, y(0)+\delta_{1}\right]$ then $X$ satisfies eq. $(h)$ on $\left(-\infty, 1-\theta_{-}\right]$. It follows that there exists a unique solution of $(h)$ defined on $\mathbb{R}$ which coincides with $X$ on $\left(-\infty, 1-\theta_{-}\right]$. We denote this solution by $z(\cdot, h)$. Obviously, $\operatorname{dist}\left(z(\cdot, h)_{t}, \mathcal{O}\right) \longrightarrow 0$ as $t \longrightarrow-\infty$, and $z(\cdot, h)_{0}=\varphi$. Similarly, if $h$ is $C^{1}$, we use the notation $w(\cdot, h):[-1, \infty) \longrightarrow \mathbb{R}$ for the solution of the variational equation $(h, z(\cdot, h))$ with initial value $w(\cdot, h)_{0}=\omega$, and we have $w(\cdot, h)=w(\cdot, g)$ on $\left[-1,1-\theta_{-}\right]$. (Here, $\omega$ is as in parts $\mathrm{g}$ ) and $\mathrm{h}$ ) of Proposition 3.4.) 
We want to find a modification $g^{*}$ of $g$ such that the phase curve of $z\left(\cdot, g^{*}\right)$ is homoclinic to $\mathcal{O}$. Further, we want to achieve that the solutions $\dot{z}\left(\cdot, g^{*}\right)$ and $w\left(\cdot, g^{*}\right)$ of the variational equation $\left(g^{*}, z\left(\cdot, g^{*}\right)\right)$ satisfy the assumptions of Theorem 2.9.

Remark 4.1. Let $\tilde{g} \in C^{1}(\mathbb{R}, \mathbb{R})$ be a modification of $g$ on $\mathbb{R} \backslash\left[-y(0)-1, y(0)+\delta_{1}\right]$. If there exists $t^{*}>0$ with $z(\cdot, \tilde{g})_{t^{*}} \in W^{s}\left(y_{0}, P, N_{\iota}\right)$ then the solution $z(\cdot, \tilde{g})$ of eq. $(\tilde{g})$ has a phase curve homoclinic to the orbit of $y$.

Proof. Set

$$
\psi:=z(\cdot, \tilde{g})_{t^{*}} \in W^{s}\left(y_{0}, P, N_{\iota}\right)=W^{s}\left(y_{0}, P, N_{k_{1} \delta_{1}}\right) .
$$

From Proposition 3.1,b), we know that the solution $x^{\psi}:[-1, \infty) \longrightarrow \mathbb{R}$ of eq. $(g)$ with $x_{0}^{\psi}=\psi$ satisfies $x_{t}^{\psi} \in \mathcal{O}+C\left(\delta_{1}\right)$ for $t \geq 0$, so $x^{\psi}$ is also a solution of $(\tilde{g})$. It follows that $z\left(t_{*}+t, \tilde{g}\right)=x^{\psi}(t)$ for $t \geq 0$, so $\operatorname{dist}(z(\cdot, \tilde{g}) t, \mathcal{O}) \longrightarrow 0$ for $t \longrightarrow \infty$.

The next statement is an improved version of Proposition 2.2 from [L-WW2] which helps to avoid zeroes of the derivative in the construction of nonlinearities.

Proposition 4.2. Let $a, b \in \mathbb{R}, a<b$, and $\zeta \in C^{0}([a, b], \mathbb{R})$. Set $m:=\min \zeta$, and $M:=\max \zeta$. Assume $m<M$ and that

$$
\mu(\{s \in[a, b]: \zeta(s) \in\{m, M\}\})=0,
$$

where $\mu$ denotes the Lebesgue measure. Let $K>0, B>0, M_{0} \in(0, B), \alpha_{0}>$ $0, \alpha_{1}>0, M_{1}<0$ and $\eta>0$ be given such that

$$
M_{0}(b-a)<K<B(b-a) \quad \text { and } \quad \alpha_{0}(b-a)<K .
$$

Then there exists $r \in\left(0, \frac{M-m}{2}\right)$ and a continuous map

$$
\begin{aligned}
\left(0, \alpha_{0}\right] \times\left(0, \alpha_{1}\right] & \longrightarrow\left(C^{0}([m, M], \mathbb{R}),||_{\infty}\right), \\
\left(m_{0}, m_{1}\right) & \mapsto h\left(\cdot, m_{0}, m_{1}\right)
\end{aligned}
$$

such that, for every $\left(m_{0}, m_{1}\right) \in\left(0, \alpha_{0}\right] \times\left(0, \alpha_{1}\right]$, the function $h=h\left(\cdot, m_{0}, m_{1}\right)$ has the following properties:

(i) $h \in C^{1}([m, M], \mathbb{R})$;

(ii) $h(m)=m_{0}, h^{\prime}(m)=m_{1}, h(M)=M_{0}, h^{\prime}(M)=M_{1}$;

(iii) $0<h(x)<B$ for $x \in[m, M], h^{\prime}((m+M) / 2)=0$, and

$$
h^{\prime}(x)>0 \text { for } x \in[m,(m+M) / 2), \quad h^{\prime}(x)<0 \text { for } x \in((m+M) / 2, M] .
$$

(iv) $\int_{a}^{b} h(\zeta(s)) d s=K$;

(v) $h(x) \geq \frac{K}{2(b-a)}$ for $x \in[m+r, M-r]$;

$$
\int_{\substack{s \in[a, b] \\ \zeta(s) \in[m, m+r]}} h(\zeta(s)) d s \leq \eta, \quad \int_{\substack{s \in[a, b] \\ \zeta(s) \in[M-r, M]}} h(\zeta(s)) d s \leq \eta .
$$

Proof. Take a function $\chi \in B C^{1}(\mathbb{R}, \mathbb{R})$ with $\chi(x)=0$ for $x \leq 0, \dot{\chi}>0$ on $(0,1)$ and $\chi(x)=1$ for $x \geq 1$. It follows from (4.2) that there exist $\lambda_{1}, \lambda_{2}>0$ with

$$
\begin{gathered}
\max \left\{\alpha_{0}, M_{0}\right\}<\lambda_{1}<\lambda_{2}<B, \text { and } \\
\frac{K}{2}<\lambda_{1}(b-a)<K<\lambda_{2}(b-a) .
\end{gathered}
$$


Choose a function $\beta \in C^{1}([m, M], \mathbb{R})$ with $\beta(m)=\beta^{\prime}(m)=\beta^{\prime}(M)=\beta(M)=0$ and with $\beta^{\prime}(x)>0$ for $x \in(m,(m+M) / 2), \beta^{\prime}(x)<0$ for $x \in((m+M) / 2, M)$. Choose $\rho_{0} \in(0,(M-m) / 2]$ such that

$$
\lambda_{2}+\rho_{0}|\beta|_{C^{0}}<B .
$$

For $m_{0} \in\left(0, \alpha_{0}\right], m_{1} \in\left(0, \alpha_{1}\right], \rho \in\left(0, \rho_{0}\right]$ and $\lambda \in \mathbb{R}$, we define a function $f_{\rho, \lambda}=f_{\rho, \lambda, m_{0}, m_{1}}:[m, M] \longrightarrow \mathbb{R}$,

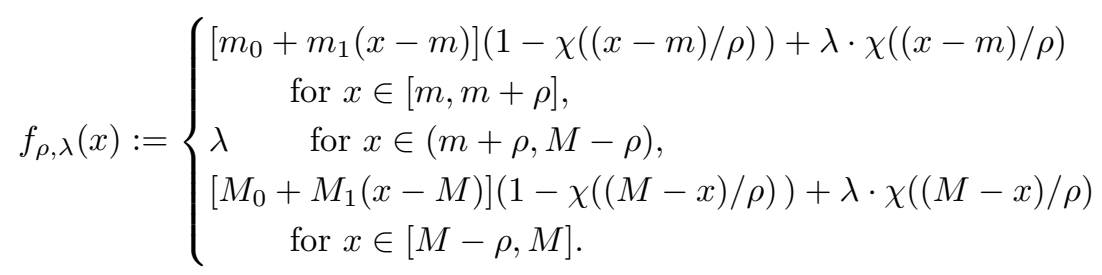

Set $g_{\rho, \lambda}:=g_{\rho, \lambda, m_{0}, m_{1}}:=f_{\rho, \lambda, m_{0}, m_{1}}+\rho \beta$ for $\rho, \lambda, m_{0}, m_{1}$ as above. Note that both $f_{\rho, \lambda}$ and $g_{\rho, \lambda}$ are in $C^{1}([m, M], \mathbb{R})$ and satisfy the boundary conditions that are imposed on the function $h$ in assertion (ii).

(4.5) Claim: For $\lambda>0$ and $\rho \in\left(0, \rho_{0}\right]$ with

$$
m_{0}+m_{1} \rho<\lambda \text { and } M_{0}+\left|M_{1}\right| \rho<\lambda,
$$

the function $g:=f_{\rho, \lambda, m_{0}, m_{1}}$ satisfies

$$
\left.g^{\prime}(x)>0 \text { for } x \in[m,(m+M) / 2), \quad g^{\prime}(x)<0 \text { for } x \in((m+M) / 2), M\right] .
$$

Proof. Let $\lambda>0$ and $\rho \in\left(0, \rho_{0}\right]$ satisfy the assumptions of the claim. First, $g^{\prime}(m)=f_{\rho, \lambda}^{\prime}(m)=m_{1}>0$. For $x \in(m, m+\rho)$, one has with $\xi:=(x-m) / \rho$ that

$$
\begin{aligned}
g^{\prime}(x)= & m_{0}\left(-\chi^{\prime}(\xi) / \rho\right)+\underbrace{m_{1}(1-\chi(\xi))}_{\geq 0} \\
& +m_{1}(x-m)\left(-\chi^{\prime}(\xi) / \rho\right)+\lambda \chi^{\prime}(\xi) / \rho+\rho \beta^{\prime}(x) \\
\geq & \left(\lambda-m_{0}\right) \chi^{\prime}(\xi) / \rho-m_{1}(x-m) \chi^{\prime}(\xi) / \rho+\rho \beta^{\prime}(x) \\
\geq & \frac{\lambda-m_{0}-m_{1} \rho}{\rho} \chi^{\prime}(\xi)\left(\text { note that } \beta^{\prime}>0 \text { on }(m, m+\rho) \subset(m,(m+M) / 2)\right) \\
> & 0(\text { from }(4.6) \text { and the choice of } \chi) .
\end{aligned}
$$

For $x \in[m+\rho,(m+M) / 2)$ respectively $x \in((m+M) / 2, M-\rho]$, we have $g^{\prime}(x)=$ $\rho \beta^{\prime}(x)>0$ respectively $\ldots<0$

For $x \in(M-\rho, M)$ and $\xi:=(M-x) / \rho$,

$$
\begin{aligned}
g^{\prime}(x) & =M_{0} \chi^{\prime}(\xi) / \rho+\underbrace{M_{1}(1-\chi(\xi))}_{\leq 0} \\
& +M_{1}(x-M) \chi^{\prime}(\xi) / \rho-\lambda \chi^{\prime}(\xi) / \rho+\underbrace{\rho \beta^{\prime}(x)}_{\leq 0} \\
& \leq \frac{M_{0}+\left|M_{1}\right| \rho-\lambda}{\rho} \chi^{\prime}(\xi)<0 .
\end{aligned}
$$

Finally, $g^{\prime}(M)=M_{1}<0$. The claim is proved.

According to (4.3), we can choose $\rho_{1} \in\left(0, \rho_{0}\right]$ such that $\max \left\{\alpha_{0}+\alpha_{1} \rho_{1}, M_{0}+\right.$ $\left.\left|M_{1}\right| \rho_{1}\right\}<\lambda_{1}$. Then, for $m_{0} \in\left(0, \alpha_{0}\right], m_{1} \in\left(0, \alpha_{1}\right], \lambda \in\left[\lambda_{1}, \lambda_{2}\right]$ and $\rho \in\left(0, \rho_{1}\right]$, 
condition (4.6) is satisfied. Claim (4.5), the definition of $g_{\rho, \lambda}$, and inequality (4.4) show that we have

$$
\left|g_{\rho, \lambda, m_{0}, m_{1}}\right|_{C^{0}} \leq g_{\rho, \lambda, m_{0}, m_{1}}((m+M) / 2) \leq \lambda+\rho|\beta|_{C^{0}} \leq \lambda_{2}+\rho_{0}|\beta|_{C^{0}}<B .
$$

Set $\epsilon_{1}:=K-\lambda_{1}(b-a), \epsilon_{2}:=\lambda_{2}(b-a)-K$. For $\rho \in\left(0, \rho_{1}\right]$, we set $E_{\rho}:=\{s \in$ $[a, b]: \zeta(s) \in[m, m+\rho] \cup[M-\rho, M]\}$. It follows from condition (4.1) and the $\sigma$-continuity of the Lebesgue measure that there exists $\rho_{2} \in\left(0, \rho_{1}\right]$ such that for $\rho \in\left(0, \rho_{2}\right]$ one has

$$
\mu\left(E_{\rho}\right)<\min \left\{\eta / B, \frac{\min \left\{\epsilon_{1}, \epsilon_{2}\right\}}{3\left(B+\lambda_{2}(b-a)+\lambda_{2}\right)}\right\} .
$$

Choose $\rho_{3} \in\left(0, \rho_{2}\right]$ such that

$$
\rho_{3}(b-a)|\beta|_{C^{0}}<\frac{\min \left\{\epsilon_{1}, \epsilon_{2}\right\}}{3} .
$$

Let now $\rho \in\left(0, \rho_{3}\right], \lambda \in\left[\lambda_{1}, \lambda_{2}\right], m_{0} \in\left(0, \alpha_{0}\right], m_{1} \in\left(0, \alpha_{1}\right]$. Using (4.7) and (4.8), we obtain

$$
\begin{aligned}
& \left|\int_{a}^{b} g_{\rho, \lambda, m_{0}, m_{1}}(\zeta(s)) d s-\lambda(b-a)\right| \\
& \leq \mu\left(E_{\rho}\right)(B+\lambda(b-a)) \\
& +\left|\int_{s \in[a, b] \backslash E_{\rho}} g_{\rho, \lambda, m_{0}, m_{1}}(\zeta(s)) d s-\lambda(b-a)\right| \\
& \leq \frac{\min \left\{\epsilon_{1}, \epsilon_{2}\right\}}{3}+\left|\int_{s \in[a, b] \backslash E_{\rho}}[\lambda+\rho \beta(\zeta(s))] d s-\lambda(b-a)\right|
\end{aligned}
$$

(use (4.8) and the definition of $g_{\rho, \lambda, m_{0}, m_{1}}$ )

$$
\begin{aligned}
& =\frac{\min \left\{\epsilon_{1}, \epsilon_{2}\right\}}{3}+\left|\lambda\left(-\mu\left(E_{\rho}\right)\right)+\int_{s \in[a, b] \backslash E_{\rho}} \rho \beta(\zeta(s)) d s\right| \\
& \leq \frac{\min \left\{\epsilon_{1}, \epsilon_{2}\right\}}{3}+\lambda_{2} \mu\left(E_{\rho}\right)+\rho(b-a)|\beta|_{C^{0}} \\
& <\frac{\min \left\{\epsilon_{1}, \epsilon_{2}\right\}}{3}+\frac{\min \left\{\epsilon_{1}, \epsilon_{2}\right\}}{3}+\frac{\min \left\{\epsilon_{1}, \epsilon_{2}\right\}}{3}=\min \left\{\epsilon_{1}, \epsilon_{2}\right\} .
\end{aligned}
$$

Consequently, we have for $m_{0} \in\left(0, \alpha_{0}\right], m_{1} \in\left(0, \alpha_{1}\right]$ that

$$
\int_{a}^{b} g_{\rho_{3}, \lambda_{1}, m_{0}, m_{1}}(\zeta(s)) d s<K<\int_{a}^{b} g_{\rho_{3}, \lambda_{2}, m_{0}, m_{1}}(\zeta(s)) d s .
$$

The definition of the functions $g_{\rho_{3}, \lambda, m_{0}, m_{1}}$ shows that the map

$$
\gamma:\left[\lambda_{1}, \lambda_{2}\right] \times\left[0, \alpha_{0}\right] \times\left[0, \alpha_{1}\right] \ni\left(\lambda, m_{0}, m_{1}\right) \mapsto g_{\rho_{3}, \lambda, m_{0}, m_{1}} \in\left(C^{0}([m, M], \mathbb{R}),||_{C^{0}}\right)
$$

is continuous. It follows that the map

$$
j:\left[\lambda_{1}, \lambda_{2}\right] \times\left[0, \alpha_{0}\right] \times\left[0, \alpha_{1}\right] \longrightarrow \mathbb{R}, \quad\left(\lambda, m_{0}, m_{1}\right) \mapsto \int_{a}^{b} g_{\rho_{3}, \lambda, m_{0}, m_{1}}(\zeta(s)) d s
$$


is continuous. $j$ is differentiable w.r. to the first argument, and one has

$$
\begin{aligned}
\partial_{1} j\left(\lambda, m_{0}, m_{1}\right)= & \int_{s \in[a, b], \zeta(s) \in\left[m, m+\rho_{3}\right]} \chi\left((\zeta(s)-m) / \rho_{3}\right) d s \\
& +\int_{s \in[a, b], \zeta(s) \in\left[M-\rho_{3}, M\right]} \chi\left((M-\zeta(s)) / \rho_{3}\right) d s+\int_{s \in[a, b] \backslash E_{\rho}} 1 d s \\
& \geq \mu\left([a, b] \backslash E_{\rho}\right)>0 .
\end{aligned}
$$

It follows from (4.9) and (4.10) that for all $\left(m_{0}, m_{1}\right) \in\left(0, \alpha_{0}\right] \times\left(0, \alpha_{1}\right]$ there exists a unique $\lambda\left(m_{0}, m_{1}\right)$ with $j\left(\lambda\left(m_{0}, m_{1}\right), m_{0}, m_{1}\right)=K$. Further, (4.10) and the Implicit Function Theorem imply that the map $\lambda:\left(m_{0}, m_{1}\right) \mapsto \lambda\left(m_{0}, m_{1}\right)$ is continuous. Set now

$$
h\left(\cdot, m_{0}, m_{1}\right):=g_{\rho_{3}, \lambda\left(m_{0}, m_{1}\right), m_{0}, m_{1}} \text { for }\left(m_{0}, m_{1}\right) \in\left(0, \alpha_{0}\right] \times\left(0, \alpha_{1}\right],
$$

and set $r:=\rho_{3}$. Continuity of $\lambda(\cdot, \cdot)$ and of the map $\gamma$ above imply that the map $\left(m_{0}, m_{1}\right) \mapsto h\left(\cdot, m_{0}, m_{1}\right) \in\left(C^{0}([m, M], \mathbb{R}),||_{C^{0}}\right)$ is continuous.

We check the asserted properties (i)-(vi) now: Properties (i) and (ii) are clear from the definition of the functions $g_{\rho, \lambda}$. Property (iii) follows from (4.7) and from Claim (4.5). Property (iv) holds, since

$$
\int_{a}^{b} h\left(\zeta(s), m_{0}, m_{1}\right) d s=j\left(\lambda\left(m_{0}, m_{1}\right), m_{0}, m_{1}\right)=K .
$$

Property (v) follows from the definition of the $g_{\rho, \lambda}$ and from the first estimate in the second line of (4.3). Property (vi) follows from property (iii) together with the estimate $B \mu\left(E_{\rho}\right) \leq \eta($ see $(4.8))$.

We now construct the first modification $g_{1}$ of $g$, which will steer the solution $z\left(\cdot, g_{1}\right)$ to negative values away from the range of the periodic solution $y$. Parallel to controlling the behavior of $z\left(\cdot, g_{1}\right)$, we have to keep track of properties of $w\left(\cdot, g_{1}\right)$, which will finally make the proof of transversality possible. From now on, Figure 1 should be helpful in following the construction. (See figures at end of paper.)

Proposition 4.3. There exists a modification $g_{1}$ of $g$ on $\left[y(0)+\delta_{1}, \infty\right)$ with the following properties:

a) $g_{1} \in B C^{1}(\mathbb{R}, \mathbb{R})$, and $g_{1}$ satisfies $(N F)$;

b) $g_{1}^{\prime}<0$ on $[0, \infty)$;

c) $z\left(1, g_{1}\right)<0$, and $z\left(\cdot, g_{1}\right)$ has a unique zero $t_{0}$ in $(1-\delta, 1)$.

Setting $t_{\min }:=t_{0}+1$, we have

d) $\dot{z}\left(t, g_{1}\right)<0$ for $t \in\left(0, t_{\min }\right)$ and $\dot{z}\left(t, g_{1}\right)>0$ for $t \in\left(t_{\min }, 2+\theta_{+}\right]$;

e) $z\left(1+\theta_{+}, g_{1}\right) \leq-y(0)-1$;

f) $w\left(t_{\min }, g_{1}\right)<0$.

Proof. Choose $\eta \in B C^{1}(\mathbb{R}, \mathbb{R})$ with $\eta(r)=0$ for $r \leq y(0)+\delta_{1}$ and with $\dot{\eta}(r)<0$ for $r>y(0)+\delta_{1}$. Set $g_{\lambda}:=g+\lambda \cdot \eta$ for $\lambda>0$. The functions $g_{\lambda}$ satisfy (NF), and $g_{\lambda}^{\prime}<0$ on $[0, \infty)$. The solutions $z\left(\cdot, g_{\lambda}\right)$ and $w\left(\cdot, g_{\lambda}\right)$ are defined for $\lambda>0$, and $z\left(t, g_{\lambda}\right)=X(t)$ for $t \in\left(-\infty, 1-\theta_{-}\right], \quad w\left(t, g_{\lambda}\right)=w(t, g)$ for $t \in\left[-1,1-\theta_{-}\right]$. Since 
$\theta_{-} \leq \delta$, we have $z\left(1-\delta, g_{\lambda}\right)=X(1-\delta)$. Since $\left|\varphi-y_{0}\right|=k_{3} \delta / 2$ and $\delta \leq \hat{\delta} \leq \bar{\delta}$, we see from part c) of Proposition 3.1 that $X(1-\delta) \geq k_{2} \delta>0$. Hence we have

$$
z\left(1-\delta, g_{\lambda}\right)>0 \text {. }
$$

Now

$$
\begin{aligned}
z\left(1, g_{\lambda}\right) & =z\left(1-\delta, g_{\lambda}\right)+\int_{-\delta}^{0}(g+\lambda \eta)(X(s)) d s \\
& \leq X(1-\delta)+\int_{-\delta}^{0} g(X(s)) d s+\lambda \int_{-\theta_{-}}^{0} \eta(X(s)) d s \\
& =X(1)+\lambda \int_{-\theta_{-}}^{0} \eta(X(s)) d s .
\end{aligned}
$$

The last integral is strictly negative, because the interval $X\left(\left[-\theta_{-}, 0\right]\right)=[y(0)+$ $\left.\delta_{1}, \varphi(0)\right]$ does not consist of one point only. Hence we obtain

$$
\lim _{\lambda \longrightarrow \infty} z\left(1, g_{\lambda}\right)=-\infty
$$

For $t \in\left(1,1+\theta_{+}\right)$, we have

$$
\dot{z}\left(t, g_{\lambda}\right) \in(g+\lambda \eta)\left(X\left[\left(0, \theta_{+}\right)\right]\right)=(g+\lambda \eta)\left[\left(\left(y(0)+\delta_{1}, \varphi(0)\right)\right] \subset(-\infty, 0],\right.
$$

so that also

$$
\lim _{\lambda \longrightarrow \infty} z\left(1+\theta_{+}, g_{\lambda}\right)=-\infty
$$

There exists $\lambda_{0}>0$ such that for $\lambda>\lambda_{0}$ one has

$$
z\left(1, g_{\lambda}\right)<0, \quad z\left(1+\theta_{+}, g_{\lambda}\right)<-y(0)-1
$$

Similarly, using property h) from Proposition 3.4 , we obtain $\dot{w}\left(t, g_{\lambda}\right)<0$ on $[1-$ $\left.\theta_{-}, 1+\theta_{+}\right]$, and

$$
\lim _{\lambda \longrightarrow \infty} w\left(1, g_{\lambda}\right)=-\infty, \quad \lim _{\lambda \longrightarrow \infty} w\left(1+\theta_{+}, g_{\lambda}\right)=-\infty .
$$

Note that with $W:=\sup \left\{|w(s, g)|:-1 \leq s \leq 1-\theta_{-}\right\}$, we have $W \geq \omega(0)>0$, and

$$
\sup \left\{\left|w\left(s, g_{\lambda}\right)\right|:-1 \leq s \leq 1-\theta_{-}\right\}=W \text { for all } \lambda>0 .
$$

Choose $\lambda_{1} \geq \lambda_{0}$ such that, for all $\lambda \geq \lambda_{1}$,

$$
\begin{aligned}
& \left|w\left(1+\theta_{+}, g_{\lambda}\right)\right|=\max \left\{\left|w\left(s, g_{\lambda}\right)\right|:-1 \leq s \leq 1+\theta_{+}\right\}, \text {and } \\
& w\left(1+\theta_{+}, g_{\lambda}\right) \leq-3|g|_{C^{1}} W .
\end{aligned}
$$

For $\lambda>0$, property (NF) shows that $z\left(\cdot, g_{\lambda}\right)$ is strictly decreasing on $[0,1]$, and therefore $z\left(t, g_{\lambda}\right) \leq z\left(\theta_{+}, g_{\lambda}\right)=y(0)+\delta_{1}$ for $t \in\left[\theta_{+}, 1\right]$. It follows that

$$
g_{\lambda}\left(z\left(t-1, g_{\lambda}\right)\right)=g\left(z\left(t-1, g_{\lambda}\right)\right) \text { for } t \in\left[1+\theta_{+}, 2\right] .
$$

For $t \in\left[1+\theta_{+}, 2-\theta_{-}\right]$, we obtain the following estimate from the definition of $W$ :

$$
\left|\dot{w}\left(t, g_{\lambda}\right)\right|=\left|g^{\prime}\left(z\left(t-1, g_{\lambda}\right)\right) w\left(t-1, g_{\lambda}\right)\right| \leq|g|_{C^{1}} W .
$$


For $t \in\left[2-\theta_{-}, 2\right]$ and $\lambda \geq \lambda_{1}$ we have, using (4.13), the estimate

$$
\begin{aligned}
\left|\dot{w}\left(t, g_{\lambda}\right)\right| & =\left|g^{\prime}\left(z\left(t-1, g_{\lambda}\right)\right) w\left(t-1, g_{\lambda}\right)\right| \\
& \leq|g|_{C^{1}} \sup \left\{\left|w\left(s, g_{\lambda}\right)\right|: s \in\left[1-\theta_{-}, 1\right]\right\} \\
& =|g|_{C^{1}}\left|w\left(1+\theta_{+}, g_{\lambda}\right)\right| .
\end{aligned}
$$

Set now $g_{1}:=g_{\lambda_{1}}$. Properties a) and b) follow from $\dot{\eta} \leq 0$ and from $g^{\prime}<0$. The inequality in c) follows from (4.12). We see from (4.11) and (4.12) that $z\left(\cdot, g_{1}\right)$ has a zero $t_{0} \in(1-\delta, 1)$. This zero is unique since $\dot{z}\left(\cdot, g_{1}\right)<0$ on $(0,1]$. It follows now from (NF) that, with $t_{\text {min }}:=t_{0}+1, \quad \dot{z}\left(t, g_{1}\right)<0$ for $t \in\left(0, t_{\min }\right)$. Since $t_{0} \in(1-\delta, 1)$, we have $z\left(t, g_{1}\right)<0$ for $t \in\left(t_{0}, t_{\text {min }}\right) \supset\left(t_{0}, 2-\delta\right) \supset\left(t_{0}, 1+\theta_{+}\right]$, and therefore $\dot{z}\left(t, g_{1}\right)>0$ for $t \in\left(t_{\min }, 2+\theta_{+}\right]$. Assertion $\left.\mathrm{d}\right)$ is proved. Assertion e) follows from (4.12).

Proof of assertion $\mathrm{f})$ : For $t \in\left[2-\delta, 2-\theta_{-}\right]$, we have from the second line of (4.13), from (4.14) and eq. $\left(g_{1}, z\left(\cdot, g_{1}\right)\right)$ that

$$
\begin{aligned}
w\left(t, g_{1}\right) & \leq w\left(1+\theta_{+}, g_{1}\right)+|g|_{C^{1}} W\left(2-\theta_{-}-\left(1+\theta_{+}\right)\right) \\
& \leq w\left(1+\theta_{+}, g_{1}\right)+|g|_{C^{1}} W \\
& \leq \frac{2}{3} w\left(1+\theta_{+}, g_{1}\right)=-\frac{2}{3}\left|w\left(1+\theta_{+}, g_{1}\right)\right|<0 .
\end{aligned}
$$

For $t \in\left[2-\theta_{-}, 2\right]$, we obtain from (4.15) and from $\theta_{-} \leq \delta \leq 1 / 3|g|_{C^{1}}$ (see (3.7)(iii)) that

$$
\begin{aligned}
w\left(t, g_{1}\right) & \leq w\left(2-\theta_{-}, g_{1}\right)+\theta_{-}|g|_{C^{1}}\left|w\left(1+\theta_{+}, g_{1}\right)\right| \\
& \leq-\frac{2}{3}\left|w\left(1+\theta_{+}, g_{1}\right)\right|+\frac{1}{3}\left|w\left(1+\theta_{+}, g_{1}\right)\right|=-\frac{1}{3}\left|w\left(1+\theta_{+}, g_{1}\right)\right|<0 .
\end{aligned}
$$

Hence $w\left(t, g_{1}\right)<0$ for $t \in[2-\delta, 2]$. Recalling that $t_{0} \in(1-\delta, 1)$, we obtain assertion f).

We set $z_{0}:=z\left(1+\theta_{+}, g_{1}\right)$ (then $\left.z_{0} \leq-y(0)-1\right)$, and $z_{4}:=z\left(2-\delta, g_{1}\right)$. (We define numbers $z_{1}, z_{2}, z_{3}$ later.)

Corollary 4.4. For $t \in\left[2-\delta, 2+\theta_{+}\right]$, one has

$$
z\left(t, g_{1}\right) \leq z_{4}+2 \delta|g|_{C^{0}} \leq z_{0}-y(0) / 8<0 .
$$

Proof. For $t \in\left[1+\theta_{+}, 2-\delta\right]$, we have $t-1 \in\left[\theta_{+}, 1-\delta\right] \subset\left[\theta_{+}, 1-\theta_{-}\right]$, so we obtain from Proposition 3.4,c) and d) that $z\left(t-1, g_{1}\right)=X(t-1) \leq y(0)+\delta_{1}$. Using Proposition 3.4,f) one gets

$$
\left|\dot{z}\left(t, g_{1}\right)-\dot{y}(t)\right|=|g(X(t-1))-g(y(t-1))| \leq y(0) / 4 \text { for } t \in\left[1+\theta_{+}, 2-\delta\right] .
$$

Recalling that $\dot{y}<0$ on $[1,2)$, that $\theta_{+} \leq \delta$, and using inequality (3.7)(i), we get

$$
\begin{aligned}
z_{4} & =z\left(2-\delta, g_{1}\right) \leq z\left(1+\theta_{+}, g_{1}\right)+y(2-\delta)-y\left(1+\theta_{+}\right)+y(0) / 4 \\
& \leq z_{0}+y(2-\delta)-y(1+\delta)+y(0) / 4 \\
& \leq z_{0}-y(0) / 4
\end{aligned}
$$

Proposition $4.3, \mathrm{~d})$ shows that

$$
z\left(t, g_{1}\right) \leq z\left(2-\delta, g_{1}\right)=z_{4} \text { for } t \in\left[2-\delta, t_{\min }\right] .
$$


For $t \in\left[t_{\min }, 2+\theta_{+}\right]$, we have $z\left(t-1, g_{1}\right) \in\left[z\left(1+\theta_{+}, g_{1}\right), 0\right]=\left[z_{0}, 0\right]$ and $\left|\dot{z}\left(t, g_{1}\right)\right|=$ $\left|g\left(z\left(t-1, g_{1}\right)\right)\right| \leq|g|_{C^{0}}$. For these $t$, it follows from the above estimate that

$$
\begin{aligned}
z\left(t, g_{1}\right) & \leq z\left(t_{\min }, g_{1}\right)+\left(2+\theta_{+}-t_{\min }\right)|g|_{C^{0}} \\
& \leq z_{4}+((2+\delta)-(2-\delta))|g|_{C^{0}} \\
& =z_{4}+2 \delta|g|_{C^{0}} .
\end{aligned}
$$

Combining (4.17) and (4.18), we obtain the first estimate of the assertion. The second estimate follows from (4.16) together with the choice of $\delta$, namely, from (3.7),(ii). The property $z_{0}-y(0) / 8<0$ is clear.

Recall the functions $\psi_{s}(s \in[0,1])$ from the end of Section 3. Our aim is to find a family $\left\{g_{s}\right\}_{s \in[0,1]}$ of modifications of $g$ such that, for an appropriate time $t^{*}$, the segment $z\left(\cdot, g_{s}\right)_{t^{*}}$ is close to $\psi_{s}$, for all $s \in[0,1]$. The construction of such nonlinearities in the next lemma includes an application of Proposition 4.2. To prepare this application, we need some notation. We know from Proposition 4.3 that $\dot{z}\left(t, g_{1}\right)<0$ for $t \in\left(0, t_{0}+1\right)$, and $\left(0, t_{0}+1\right) \supset\left[1+\theta_{+}, 2-\delta\right]$. Consequently, $z\left(\cdot, g_{1}\right) \mid\left[1+\theta_{+}, 2-\delta\right]$ has a $C^{1}$ inverse function

$$
\tau:\left[1+\theta_{+}, 2-\delta\right] \longrightarrow\left[z\left(2-\delta, g_{1}\right), z\left(1+\theta_{+}, g_{1}\right)\right]=\left[z_{4}, z_{0}\right] \subset \mathbb{R} .
$$

Recall that, for all $s \in[0,1]$, we have $\dot{\psi}_{s}(t)>0$ for $t \in[-1,0), \ddot{\psi}_{s}(t)<0$ for $t \in[-1,0]$. Set

$$
\begin{gathered}
\alpha_{0}:=\max _{s \in[0,1]} \dot{\psi}_{s}(-1)>0 ; \quad \alpha_{1}:=\max _{s \in[0,1], t \in[0,1 / 4]} \frac{\ddot{\psi}_{s}(-1)}{\dot{z}\left(1+\theta_{+}+t, g_{1}\right)}>0 ; \\
M_{0}:=g\left(z\left(1+\theta_{+}, g_{1}\right)\right)=g\left(z_{0}\right)>0 ; \quad M_{1}:=g^{\prime}\left(z_{0}\right)<0 ; \\
\eta:=y(0) / 16, \quad K:=-z\left(2+\theta_{+}, g_{1}\right) .
\end{gathered}
$$

(From Corollary 4.4, we have $K>0$.) Choose now $\tilde{\theta} \in(0,1 / 4)$ such that the following inequalities hold:

$$
\begin{gathered}
\tilde{\theta} \leq \delta ; \\
z\left(1+\theta_{+}+\tilde{\theta}, g_{1}\right) \geq z\left(1+\theta_{+}, g_{1}\right)-y(0) / 16=z_{0}-y(0) / 16 ; \\
\tilde{\theta}\left[M_{2}+\frac{2(K+1)}{K}|g|_{C_{0}}+\max \left\{|g|_{C^{0}}, M_{2}\right\}+|g|_{C^{0}}\right] \leq \frac{k_{4} k_{1} k_{3} \delta}{64} ; \\
M_{0} \tilde{\theta}<K, \quad \alpha_{0} \tilde{\theta}<K .
\end{gathered}
$$

Set $B:=(K+1) / \tilde{\theta}$. Finally, define $z_{3}:=z\left(1+\theta_{+}+\tilde{\theta}, g_{1}\right)$. Note that the choice of $\alpha_{1}$ and the inequality $\tilde{\theta}<1 / 4$ imply that, for all $s \in[0,1]$,

$$
0<\frac{\ddot{\psi}_{s}(-1)}{\dot{z}\left(1+\theta_{+}+\tilde{\theta}, g_{1}\right)} \leq \alpha_{1}
$$

Lemma 4.5. There exist a continuous map

$$
[0,1] \ni s \mapsto g_{s} \in\left(B C^{0}(\mathbb{R}, \mathbb{R}),||_{C^{0}}\right)
$$

and numbers $\tau_{1}, \tau_{2} \in\left(1+\theta_{+}, 1+\theta_{+}+\tilde{\theta}\right)$ and $z_{\max } \in\left(z_{3}, z_{0}\right)$ satisfying the subsequent properties for all $s \in[0,1]$. (We set $z_{i}:=z\left(\tau_{i}, g_{1}\right)(i=1,2)$.) 
a) $g_{s} \in B C^{1}(\mathbb{R}, \mathbb{R})$, and $g_{s}$ satisfies $(N F)$. $g_{s}=g_{1}$ on $\left[z_{0}, \infty\right) \supset[-y(0)-1, \infty)$, and

$$
g_{s}^{\prime}>0 \text { on }\left(-\infty, z_{\max }\right), \quad g_{s}^{\prime}<0 \text { on }\left(z_{\max }, \infty\right)
$$

b) $g_{s}\left(z_{\max }\right) \leq \frac{K+1}{\tilde{\theta}}, \quad g_{s} \geq \frac{K}{2 \tilde{\theta}}$ on $\left[z_{2}, z_{1}\right]$.

c) $\int_{1+\theta_{+}}^{\tau_{1}} g_{s}\left(z\left(t, g_{1}\right)\right) d t \leq y(0) / 16, \quad \int_{\tau_{2}}^{1+\theta_{+}+\tilde{\theta}} g_{s}\left(z\left(t, g_{1}\right)\right) d t \leq y(0) / 16$, and

$$
\int_{1+\theta_{+}}^{1+\theta_{+}+\tilde{\theta}} g_{s}\left(z\left(t, g_{1}\right)\right) d t=-z\left(2+\theta_{+}, g_{1}\right) \text {. }
$$

d) $g_{s}\left(z\left(t, g_{1}\right)\right)=\dot{\psi}_{s}\left(t-\left(2+\theta_{+}+\tilde{\theta}\right)\right)$ for $t \in\left[1+\theta_{+}+\tilde{\theta}, 2-\delta\right]$.

e) $g_{s}(x) \leq M_{2}$ for $x \leq z_{3}$.

Proof. We set $a:=1+\theta_{+}, b:=1+\theta_{+}+\tilde{\theta}$, and $\zeta:=z\left(\cdot, g_{1}\right)_{\mid[a, b]}$. We want to apply Proposition 4.2 to the function $\zeta$ and with the numbers $K, B, M_{0}, \alpha_{0}, \alpha_{1}, M_{1}$ and $\eta$ from above. Condition (4.1) is satisfied, since $\zeta$ is strictly decreasing, and we have $m=\min \zeta=z_{3}, M=\max \zeta=z_{0}$. The inequalities in (4.2) are satisfied, in view of (4.22) and the choice of $B$. Applying Proposition 4.2, we obtain a number $r \in$ $\left(0,\left(z_{0}-z_{3}\right) / 2\right)$ and a continuous map $h:\left(0, \alpha_{0}\right] \times\left(0, \alpha_{1}\right] \longrightarrow\left(C^{0}\left(\left[z_{3}, z_{0}\right], \mathbb{R}\right),||_{C^{0}}\right)$ with the properties (i)-(vi) listed in Proposition 4.2. We now define $g_{s}$ for $s \in[0,1]$. (See Figure 2.) Note that the second inequality of Corollary 4.4 and (4.20) imply $z_{4}<z_{3}$.

$$
g_{s}(x):=\left\{\begin{array}{c}
g_{1}(x) \text { for } x \geq z_{0} ; \\
h\left(x, \dot{\psi}_{s}(-1), \frac{\ddot{\psi}_{s}(-1)}{\dot{z}\left(1+\theta_{+}+\tilde{\theta}, g_{1}\right)}\right) \text { for } x \in\left[z_{3}, z_{0}\right] \\
\dot{\psi}_{s}\left(\tau(x)-\left(2+\theta_{+}+\tilde{\theta}\right)\right) \text { for } x \in\left(z_{4}, z_{3}\right) ; \\
\dot{\psi}_{s}\left(-\delta-\theta_{+}-\tilde{\theta}\right) \cdot \exp \left[\left(x-z_{4}\right) \cdot \frac{\ddot{\psi}_{s}\left(-\delta-\theta_{+}-\tilde{\theta}\right)}{\dot{\psi}_{s}\left(-\delta-\theta_{+}-\tilde{\theta}\right) \cdot \dot{z}\left(2-\delta, g_{1}\right)}\right] \\
\text { for } x \in\left(-\infty, z_{4}\right] .
\end{array}\right.
$$

Set $\tau_{1}:=\tau\left(z_{0}-r\right)$ and $\tau_{2}:=\tau\left(z_{3}+r\right)$, so that $1+\theta_{+}<\tau_{1}<\tau_{2}<1+\theta_{+}+\tilde{\theta}$. Note that $g_{s}$ is well-defined on $\left[z_{3}, z_{0}\right]$ since $\dot{\psi}_{s}(-1) \in\left(0, \alpha_{0}\right]$ and $\frac{\ddot{\psi}_{s}(-1)}{\dot{z}\left(1+\theta_{+}+\tilde{\theta}, g_{1}\right)} \in$ $\left(0, \alpha_{1}\right]$. Note also that for $x \in\left(z_{4}, z_{3}\right)$, we have $\tau(x) \in\left(1+\theta_{+}+\tilde{\theta}, 2-\delta\right)$, so $\tau(x)-\left(2+\theta_{+}+\tilde{\theta}\right) \in\left(-1,-\delta-\theta_{+}-\tilde{\theta}\right)$, which shows that $g_{s}$ is well-defined on $\left(z_{4}, z_{3}\right)$. We now prove the asserted properties of the functions $g_{s}$. For $s \in[0,1]$, let $g_{s, 1}, g_{s, 2}, g_{s, 3}$ and $g_{s, 4}$ denote the restrictions of $g_{s}$ to $I_{1}:=\left[z_{0}, \infty\right), I_{2}:=$ $\left[z_{3}, z_{0}\right], I_{3}:=\left(z_{4}, z_{3}\right), I_{4}:=\left(-\infty, z_{4}\right]$, respectively. It is clear from the definitions, from assertion (i) of Proposition 4.2, from the fact that $\psi_{s} \in C^{2}([-1,0], \mathbb{R})$, and from $C^{1}$-smoothness of $\tau$ that $g_{s, i} \in B C^{1}\left(I_{i}, \mathbb{R}\right), \quad i=1, \ldots, 4$. The map $[0,1] \ni$ 
$s \mapsto \psi_{s} \in\left(C^{2}([-1,0], \mathbb{R}),||_{C^{2}}\right)$ is continuous, and the map

$$
\begin{gathered}
E: \mathbb{R}^{+} \times \mathbb{R}^{-} \longrightarrow\left(B C^{0}\left(\left(-\infty, z_{4}\right], \mathbb{R}\right),||_{C^{0}}\right), \\
E(\alpha, \beta)(x):=\exp \left(\left(x-z_{4}\right) \frac{\beta}{\alpha \dot{z}\left(2-\delta, g_{1}\right)}\right)
\end{gathered}
$$

is continuous. In view of continuity of the map $h$, it is now obvious that the maps $[0,1] \ni s \mapsto g_{s, i} \in\left(B C^{0}\left(I_{i}, \mathbb{R}\right),||_{C^{0}}\right)$ are continuous for $i=1, \ldots, 4$.

Claim: $g_{s} \in B C^{1}(\mathbb{R}, \mathbb{R})$ for $s \in[0,1]$, and the map

$$
[0,1] \ni s \mapsto g_{s} \in\left(B C^{0}(\mathbb{R}, \mathbb{R}),||_{C^{0}}\right)
$$

is continuous.

Proof. It suffices to show that the functions $g_{s}$ are continuously differentiable at the points $z_{0}, z_{3}, z_{4}$; the continuity assertion then follows from the statement preceding the claim. Using $g_{1}=g$ on $\left(-\infty, y(0)+\delta_{1}\right)$, and property (ii) from Proposition 4.2, and recalling $M=z_{0}$, we see that

$$
\begin{aligned}
& \lim _{x \longrightarrow z_{0}+} g_{s}(x)=g\left(z_{0}\right)=M_{0}=\lim _{x \longrightarrow z_{0}-} h(x, \ldots)=\lim _{x \longrightarrow z_{0}-} g_{s}(x), \text { and } \\
& \lim _{x \longrightarrow z_{0}+} g_{s}^{\prime}(x)=g_{1}^{\prime}\left(z_{0}\right)=g^{\prime}\left(z_{0}\right)=M_{1}=\lim _{x \longrightarrow z_{0}-} \partial_{1} h(x, \ldots)=\lim _{x \longrightarrow z_{0}-} g_{s}^{\prime}(x) .
\end{aligned}
$$

Similarly,

$$
\begin{aligned}
\lim _{x \longrightarrow z_{3}+} g_{s, 2}(x) & =h\left(z_{3}, \ldots\right)=h(m, \ldots)=\dot{\psi}_{s}(-1) \\
& =\dot{\psi}_{s}\left(\tau\left(z_{3}\right)-\left(2+\theta_{+}+\tilde{\theta}\right)\right)=\lim _{x \longrightarrow z_{3}-} g_{s, 3}(x),
\end{aligned}
$$

and

$$
\begin{aligned}
\lim _{x \longrightarrow z_{3}+} g_{s, 2}^{\prime}(x) & =\partial_{1} h\left(z_{3}, \ldots\right)=\frac{\ddot{\psi}_{s}(-1)}{\dot{z}\left(1+\theta_{+}+\tilde{\theta}, g_{1}\right)} \\
& =\ddot{\psi}_{s}(-1) \cdot \tau^{\prime}\left(z_{3}\right)=\lim _{x \longrightarrow z_{3}-} g_{s, 3}^{\prime}(x)
\end{aligned}
$$

where the last equality comes from the chain rule. Finally,

$$
\begin{aligned}
\lim _{x \longrightarrow z_{4}+} g_{s, 3}(x) & =\dot{\psi}_{s}\left(\tau\left(z_{4}\right)-\left(2+\theta_{+}+\tilde{\theta}\right)\right)=\dot{\psi}_{s}\left(-\delta-\theta_{+}-\tilde{\theta}\right) \\
& =\lim _{x \longrightarrow z_{4}-} g_{s, 4}(x),
\end{aligned}
$$

and

$$
\begin{aligned}
\lim _{x \longrightarrow z_{4}+} g_{s, 3}^{\prime}(x) & =\ddot{\psi}_{s}\left(-\delta-\theta_{+}-\tilde{\theta}\right) \tau^{\prime}\left(z_{4}\right) \\
& =\frac{\ddot{\psi}_{s}\left(-\delta-\theta_{+}-\tilde{\theta}\right)}{\dot{z}\left(2-\delta, g_{1}\right)}=\lim _{x \longrightarrow z_{4}-} g_{s, 4}^{\prime}(x) .
\end{aligned}
$$

(The claim is proved.)

It follows from $0<h$ (see part (iii) of Proposition 4.2) and from $\dot{\psi}_{s}>0$ on $[-1,0)$ that $g_{s}(x)>0$ for $x \leq z_{0}$. Since $g_{1}$ satisfies $(\mathrm{NF})$, it follows that $g_{s}$ also satisfies (NF).

Proof of the remaining assertions of a): $g_{s}(x)=g_{s, 1}(x)=g_{1}(x)$ for $x \geq z_{0}$, and $g_{s, 4}^{\prime}(x)>0$ for $x \leq z_{4}, g_{s, 3}^{\prime}(x)=\ddot{\psi}_{s}(\ldots) \tau^{\prime}(x)>0$ for $x \in\left(z_{4}, z_{3}\right)$. Setting $z_{\max }:=\left(z_{0}+z_{3}\right) / 2$, it follows from part (iii) of Proposition 4.2 that $g_{s, 2}^{\prime}>0$ on 
$\left[z_{3}, z_{\max }\right), g_{s, 2}^{\prime}<0$ on $\left(z_{\max }, z_{0}\right]$. Since $g_{1}^{\prime}<0$, we have $g_{s}^{\prime}<0$ on $\left[z_{0}, \infty\right)$. Together, we obtain the asserted properties of $g_{s}^{\prime}$.

Proof of b): From Proposition 4.2,(iii), we have $g_{s}\left(z_{\max }\right)=h\left(z_{\max }, \ldots\right)<B=$ $\frac{K+1}{\tilde{\theta}}$. For $x \in\left[z_{2}, z_{1}\right]=\left[z_{3}+r, z_{0}-r\right]=[m+r, M-r]$, property (v) from Proposition 4.2 shows $g_{s}(x)=h(x, \ldots) \geq K / 2 \tilde{\theta}$.

Proof of c): $\dot{\zeta}<0$ implies that $\{s \in[a, b]: \zeta(s) \in[m, m+r]\}=\left[\tau_{2}, 1+\theta_{+}+\tilde{\theta}\right]$ and $\{s \in[a, b]: \zeta(s) \in[M-r, M]\}=\left[1+\theta_{+}, \tau_{1}\right]$. The first two estimates of assertion c) now follow from part (vi) of Proposition 4.2, the definition of $\tau_{1}$ and $\tau_{2}$, and the choice of $\eta$. The equality in assertion c) follows from part (iv) of Proposition 4.2.

Proof of d): For $t \in\left[1+\theta_{+}+\tilde{\theta}, 2-\delta\right]$, one has $z\left(t, g_{1}\right) \in\left[z_{4}, z_{3}\right]$, and

$$
g_{s}\left(z\left(t, g_{1}\right)\right)=g_{s, 3}\left(z\left(t, g_{1}\right)\right)=\dot{\psi}_{s}\left(t-\left(2+\theta_{+}+\tilde{\theta}\right)\right) .
$$

Proof of e): Recall the definition of $\psi_{s}$, and Proposition 3.3. For $x \leq z_{3}$, we have

$$
\begin{aligned}
g_{s}(x) & \leq g_{s}\left(z_{3}\right)=\dot{\psi}_{s}(-1)=s \dot{\psi}_{+}(-1)+(1-s) \dot{\psi}_{-}(-1) \\
& \leq \max \left\{\left|\psi_{+}\right|_{C^{2}},\left|\psi_{-}\right|_{C^{2}}\right\} \leq M_{2} .
\end{aligned}
$$

Together with the estimate on the solutions $z\left(\cdot, g_{1}\right)$ from Corollary 4.4, the remark below is a preparation for estimating the error in the approximation of the targets $\psi_{s}$.

Remark 4.6. For $s \in[0,1]$ and $x \in\left[z_{4}, z_{4}+2 \delta|g|_{C^{0}}\right]$, one has

$$
0<g_{s}(x) \leq 5 M_{2} c_{g} \sqrt{\delta} .
$$

Proof. Set $z_{4}^{+}:=z_{4}+2 \delta|g|_{C^{0}}$. Let $t \in[3 / 2,2-\delta]$. Since $\theta_{-} \leq \delta$, we have $z\left(\cdot, g_{1}\right)=X(\cdot)$ on $(-\infty, t-1]$. Using Proposition 3.4,c), and Proposition 3.1,c), we see that

$$
X(t-1) \in[X(1-\delta), X(1 / 2)] \subset\left[0, X\left(\theta_{+}\right)\right]=\left[0, y(0)+\delta_{1}\right]
$$

and $g=g_{1}$ on this interval. Hence

$$
\left|g_{1}^{\prime}\left(z\left(t-1, g_{1}\right)\right)\right|=\left|g^{\prime}(X(t-1))\right| \geq\left|g^{\prime}(0)\right|,
$$

since $y(0)+\delta_{1}<A$ and $g^{\prime}$ is decreasing on $[0, A]$. Using Proposition 3.4,c), the fact that $g$ is decreasing on $[0, \infty)$, and the last inequality from Proposition $3.4, a)$, we get

$$
\begin{aligned}
\left|g_{1}\left(z\left(t-2, g_{1}\right)\right)\right| & =|g(X(t-2))| \geq|g(X(-1 / 2))|=|g(\varphi(-1 / 2))| \\
& \geq|g(y(-1 / 2) / 2)| .
\end{aligned}
$$

Recall the definitions of $\gamma_{2}$ and $c_{g}$ which were given before (3.7). From eq. $\left(g_{1}\right)$, we now obtain

$$
\ddot{z}\left(t, g_{1}\right) \geq\left|g^{\prime}(0)\right||g(y(-1 / 2) / 2)|=\gamma_{2} \text { for } t \in[3 / 2,2-\delta] .
$$

Taylor expansion of $z\left(\cdot, g_{1}\right)$ at $2-\delta$ gives, for $r \in[0,1 / 2-\delta]$,

$$
z\left(2-\delta-r, g_{1}\right)=z\left(2-\delta, g_{1}\right)+\dot{z}\left(2-\delta, g_{1}\right)(-r)+\ddot{z}(2-\delta-\sigma) \cdot r^{2} / 2,
$$

for some $\sigma \in[0, r]$. Since $\dot{z}\left(2-\delta, g_{1}\right)<0$, we see from (4.24) that

$$
z\left(2-\delta-r, g_{1}\right) \geq z\left(2-\delta, g_{1}\right)+\frac{\gamma_{2}}{2} r^{2}=z_{4}+\frac{\gamma_{2}}{2} r^{2} .
$$


From the choice of $\delta$ (see (3.7),(iv)), we have that

$$
r_{+}:=2 c_{g} \sqrt{\delta} \in[0,1 / 2-\delta],
$$

and hence we have

$$
z\left(2-\delta-r_{+}, g_{1}\right) \geq z_{4}+\frac{\gamma_{2}}{2} 4 c_{g}^{2} \delta=z_{4}+2|g|_{C^{0}} \delta
$$

Recall the inverse function $\tau$ of $z\left(\cdot, g_{1}\right)_{\mid\left[1+\theta_{+}, 2-\delta\right]}$. Let now $x \in\left[z_{4}, z_{4}+2 \delta|g|_{C^{0}}\right]$.

Then Corollary 4.4 and the choice of $\tilde{\theta}$ (see (4.20)) show that

$$
x \in\left[z_{4}, z_{0}-y(0) / 8\right] \subset\left[z_{4}, z_{0}-y(0) / 16\right] \subset\left[z_{4}, z_{3}\right] .
$$

Moreover, we see from (4.25) that $\tau(x) \geq 2-\delta-r_{+}$. It follows from property (NF) of $g_{s}$ and Lemma $\left.4.5, \mathrm{~d}\right)$, that, for $s \in[0,1]$, we have

$$
\begin{aligned}
0 & <g_{s}(x)=g_{s}\left(z\left(\tau(x), g_{1}\right)\right)=\dot{\psi}_{s}\left(\tau(x)-\left(2+\theta_{+}+\tilde{\theta}\right)\right) \\
& \leq \dot{\psi}_{s}(0)+\left|\ddot{\psi}_{s}\right|_{C^{0}} \cdot\left(2+\theta_{+}+\tilde{\theta}-\tau(x)\right) .
\end{aligned}
$$

From Proposition 3.3,(i), we have $\dot{\psi}_{s}(0)=0$. With Proposition 3.3,(ii), Proposition $3.4, \mathrm{e})$, estimate (4.19) and the choice of $\delta$ (see (3.7),(iv)), one obtains

$$
\begin{aligned}
g_{s}(x) & \leq M_{2}\left(2+\theta_{+}+\tilde{\theta}-\left(2-\delta-r_{+}\right)\right)=M_{2}\left(\theta_{+}+\tilde{\theta}+\delta+r_{+}\right) \\
& \leq M_{2}\left(3 \delta+2 c_{g} \sqrt{\delta}\right) \leq 5 M_{2} c_{g} \sqrt{\delta} .
\end{aligned}
$$

We now show that the nonlinearities $g_{s}$ already lead to solutions that closely approximate the targets $\psi_{s}$. This would be sufficient in order to obtain a homoclinic solution from a shooting argument, but we still have to arrange the transversality.

Lemma 4.7. Set $t^{*}:=3+\theta_{+}+\tilde{\theta}$. For $s \in[0,1]$, we have the following properties.

a) $z\left(t, g_{s}\right)=z\left(t, g_{1}\right)$ for $t \in\left(-\infty, 2+\theta_{+}\right]$;

b) $\dot{z}\left(t, g_{s}\right)>0$ for $t \in\left(t_{\min }, 3+\theta_{+}+\tilde{\theta}\right)$;

c) With $\chi_{s}:=z\left(\cdot, g_{s}\right)_{t^{*}} \in C$, we have $\chi_{s} \in H$ and $\left|\chi_{s}-\psi_{s}\right| \leq \frac{k_{4} \iota}{2}$.

Proof. Let $s \in[0,1]$. Proof of a): From Proposition 4.3,e), we have $z_{0}=$ $z\left(1+\theta_{+}, g_{1}\right) \leq-y(0)-1$, and we know from Lemma 4.5,a) that $g_{s}=g_{1}$ on $\left[z_{0}, \infty\right)$.

Claim: $z\left(t, g_{1}\right) \geq z_{0}$ for $t \in\left(-\infty, 1+\theta_{+}\right]$.

Proof. For $t \in\left(-\infty, 1-\theta_{-}\right]$, we have from Proposition 3.4,d) that

$$
z\left(t, g_{1}\right)=X(t) \geq-y(0)-1 / 2>z_{0} .
$$

For $t \in\left[1-\theta_{-}, 1+\theta_{+}\right] \subset\left[0, t_{0}+1\right]$, Proposition 4.3,d) shows that $z\left(t, g_{1}\right) \geq$ $z\left(1+\theta_{+}, g_{1}\right)=z_{0}$. (The claim is proved.)

It follows from the above claim, from eq. $\left(g_{s}\right)$ and from $g_{s}=g_{1}$ on $\left[z_{0}, \infty\right)$ that $z\left(\cdot, g_{s}\right)=z\left(\cdot, g_{1}\right)$ on $\left(-\infty, 2+\theta_{+}\right]$.

Proof of b): Using assertion a), Proposition 4.3,d), the property $z\left(\cdot, g_{1}\right)<0$ on $\left[1+\theta_{+}, 1+\theta_{+}+\tilde{\theta}\right]$, and property $(\mathrm{NF})$ of $g_{s}$, we conclude

$$
\dot{z}\left(t, g_{s}\right)>0 \text { on }\left(t_{\mathrm{min}}, 2+\theta_{+}+\tilde{\theta}\right] .
$$


It follows from the equality in Lemma $4.5, \mathrm{c})$, from eq. $\left(g_{s}\right)$ and from assertion a) that

$$
z\left(2+\theta_{+}+\tilde{\theta}, g_{s}\right)=z\left(2+\theta_{+}, g_{1}\right)+\int_{1+\theta_{+}}^{1+\theta_{+}+\tilde{\theta}} g_{s}\left(z\left(t, g_{1}\right)\right) d t=0
$$

Combining assertion a), Proposition 4.3,d), (4.26) and (4.27), we get that $z\left(\cdot, g_{s}\right)<0$ on $\left(t_{0}, 2+\theta_{+}+\tilde{\theta}\right)$. Assertion b) now follows from (NF).

Proof of c): From (4.27), we have $\chi_{s}(-1)=0$, which means that $\chi_{s} \in H$. For $t \in\left[2+\theta_{+}+\tilde{\theta}, 3-\delta\right]=\left[t^{*}-1, t^{*}-\left(\delta+\theta_{+}+\tilde{\theta}\right)\right]$, we have from assertion a) and Lemma $4.5, \mathrm{~d})$ that

$$
\dot{z}\left(t, g_{s}\right)=g_{s}\left(z\left(t-1, g_{1}\right)\right)=\dot{\psi}_{s}\left(t-\left(3+\theta_{+}+\tilde{\theta}\right)\right) .
$$

It follows from (4.27) and (4.28) that

$$
z\left(t^{*}+t, g_{s}\right)=\psi_{s}(t) \text { for } t \in\left[-1,-\delta-\theta_{+}-\tilde{\theta}\right] .
$$

In order to prove c), it therefore suffices to prove

$$
\left|z\left(t, g_{s}\right)-\psi_{s}\left(t-t^{*}\right)\right| \leq \frac{k_{4} \iota}{2} \text { for } t \in\left[3-\delta, 3+\theta_{+}+\tilde{\theta}\right] .
$$

Proof of (4.30): For $t \in\left[3-\delta, 3+\theta_{+}+\tilde{\theta}\right]$, we have, using (4.29), that

$$
\begin{aligned}
\left|z\left(t, g_{s}\right)-\psi_{s}\left(t-t^{*}\right)\right| & \leq\left|z\left(t, g_{s}\right)-z\left(3-\delta, g_{s}\right)\right|+\left|z\left(3-\delta, g_{s}\right)-\psi_{s}\left(t-t^{*}\right)\right| \\
& =\left|z\left(t, g_{s}\right)-z\left(3-\delta, g_{s}\right)\right|+\left|\psi_{s}\left(-\delta-\theta_{+}-\tilde{\theta}\right)-\psi_{s}\left(t-t^{*}\right)\right| .
\end{aligned}
$$

For $t \in\left[2-\delta, 2+\theta_{+}+\tilde{\theta}\right]$, one has $z\left(t, g_{s}\right) \in\left[z\left(t_{\min }, g_{1}\right), 0\right]$ and therefore $\dot{z}\left(t, g_{s}\right) \geq 0$ for $t \in\left[3-\delta, 3+\theta_{+}+\tilde{\theta}\right]$. Since also $\dot{\psi}_{s} \geq 0$, we can estimate the last two terms in $(4.31)$ by $\left|z\left(3+\theta_{+}+\tilde{\theta}, g_{s}\right)-z\left(3-\delta, g_{s}\right)\right|$ and by $\left|\psi_{s}(0)-\psi_{s}\left(-\delta-\theta_{+}-\tilde{\theta}\right)\right|$, respectively. In order to prove (4.30), it therefore suffices to prove the following two estimates:

$$
\begin{gathered}
\left|z\left(3+\theta_{+}+\tilde{\theta}, g_{s}\right)-z\left(3-\delta, g_{s}\right)\right| \leq \frac{k_{4} \iota}{4}, \\
\left|\psi_{s}(0)-\psi_{s}\left(-\delta-\theta_{+}-\tilde{\theta}\right)\right| \leq \frac{k_{4} \iota}{4} .
\end{gathered}
$$

Proof of (4.32): For $t \in\left[3-\delta, 3+\theta_{+}\right]$, we have $t-1 \in\left[2-\delta, 2+\theta_{+}\right]$and $z\left(t-1, g_{s}\right)=z\left(t-1, g_{1}\right)$. Combining the first inequality from Corollary 4.4 with Remark 4.6, one obtains

$$
0<\dot{z}\left(t, g_{s}\right)=g_{s}\left(z\left(t-1, g_{1}\right)\right) \leq 5 M_{2} c_{g} \sqrt{\delta} .
$$

With the choice of $\delta$ (see $(3.7)(\mathrm{v}))$, the estimate on $\delta_{1}$ from Proposition 3.4,a), and the definition of $\iota=k_{1} \delta_{1}$ (see the end of Section 3), we obtain

$$
\begin{aligned}
& \left|z\left(3+\theta_{+}, g_{s}\right)-z\left(3-\delta, g_{s}\right)\right| \leq 5 M_{2} c_{g} \sqrt{\delta}\left(\theta_{+}+\delta\right) \\
& \leq 10 M_{2} c_{g} \delta^{3 / 2} \quad\left(\text { use } \theta_{+} \leq \delta\right) \\
& \leq \frac{k_{4} k_{3} k_{1} \delta}{64} \leq \frac{k_{4} k_{1} \delta_{1}}{8}=\frac{k_{4} \iota}{8} .
\end{aligned}
$$


Recall the numbers $\tau_{1}$ and $\tau_{2}$ from Lemma 4.5. For $t \in\left[2+\theta_{+}, \tau_{1}+1\right]$, one has $t-1 \leq \tau_{1}<2+\theta_{+}$, and in view of assertion a) and the definition of $z_{1}$ and $z_{0}$ one obtains

$$
z\left(t-1, g_{s}\right)=z\left(t-1, g_{1}\right) \in\left[z_{1}, z_{0}\right] \subset(-\infty, 0) .
$$

Hence, $g_{s}\left(z\left(t-1, g_{s}\right)\right)>0$, and Lemma 4.5,c) shows that

$$
\begin{aligned}
z\left(t, g_{s}\right) & =z\left(2+\theta_{+}, g_{s}\right)+\int_{1+\theta_{+}}^{t-1} g_{s}\left(z\left(\tau, g_{1}\right)\right) d \tau \\
& \leq z\left(2+\theta_{+}, g_{s}\right)+\int_{1+\theta_{+}}^{\tau_{1}} g_{s}\left(z\left(\tau, g_{1}\right)\right) d \tau \\
& \leq z\left(2+\theta_{+}, g_{s}\right)+y(0) / 16 \\
& =z\left(2+\theta_{+}, g_{1}\right)+y(0) / 16
\end{aligned}
$$

Combining this estimate with Corollary 4.4 and the choice of $\tilde{\theta}$ (see (4.20)), we see that

$$
z\left(t, g_{s}\right) \leq z_{0}-y(0) / 16 \leq z\left(1+\theta_{+}+\tilde{\theta}, g_{1}\right)=z_{3} .
$$

Now Lemma 4.5,e) shows

$$
g_{s}\left(z\left(t, g_{s}\right)\right) \leq M_{2} \text { for } t \in\left[2+\theta_{+}, \tau_{1}+1\right] .
$$

For $t \in\left[\tau_{2}+1,2+\theta_{+}+\tilde{\theta}\right] \subset\left[2+\theta_{+}, 2+\theta_{+}+\tilde{\theta}\right] \subset\left(t_{\mathrm{min}}, 3+\theta_{+}+\tilde{\theta}\right)$, we have from assertion b) and from (4.27) that

$$
0=z\left(2+\theta_{+}+\tilde{\theta}, g_{s}\right) \geq z\left(t, g_{s}\right) \geq z\left(\tau_{2}+1, g_{s}\right) ;
$$

with Lemma $4.5, \mathrm{c})$, one gets

$$
z\left(\tau_{2}+1, g_{s}\right)=-\int_{\tau_{2}}^{1+\theta_{+}+\tilde{\theta}} g_{s}\left(z\left(t, g_{1}\right)\right) d s \geq-y(0) / 16>-y(0) .
$$

Hence, we have

$$
\left|g_{s}\left(z\left(t, g_{s}\right)\right)\right|=\left|g\left(z\left(t, g_{s}\right)\right)\right| \leq|g|_{C^{0}}
$$

for $t \in\left[\tau_{2}+1,2+\theta_{+}+\tilde{\theta}\right]$. For $x \in(-\infty, 0] \backslash\left[z_{3}, z_{0}\right]$, Lemma 4.5,a) and Lemma 4.5,e) together with the definition of $g_{1}$ show that $\left|g_{s}(x)\right| \leq \max \left\{|g|_{C^{0}}, M_{2}\right\}$. Further,

$$
z_{0}-z_{3} \leq \int_{\theta_{+}}^{\theta_{+}+\tilde{\theta}}|g(X(t))| d t \leq|g|_{C^{0}} \tilde{\theta}
$$

For $t \in\left[\tau_{1}+1, \tau_{2}+1\right]$, we have $\dot{z}\left(t, g_{s}\right) \in g_{s}\left(\left[z_{2}, z_{1}\right]\right)$, so the second estimate of Lemma $4.5, \mathrm{~b})$ shows that $\dot{z}\left(t, g_{s}\right) \geq \frac{K}{2 \tilde{\theta}}$. Using also the first inequality of Lemma 
$4.5, \mathrm{~b})$, we obtain the following estimate.

$$
\begin{aligned}
& \left|\int_{\tau_{1}+1}^{\tau_{2}+1} g_{s}\left(z\left(t, g_{s}\right)\right) d t\right| \leq\left|\int_{\substack{\left\{t \in\left[\tau_{1}+1, \tau_{2}+1\right] \\
z\left(t, g_{s}\right) \in\left[z_{3}, z_{0}\right]\right\}}} \ldots\right|+\left|\underset{\substack{\left\{t \in\left[\tau_{1}+1, \tau_{2}+1\right] \\
z\left(t, g_{s}\right) \notin\left[z_{3}, z_{0}\right]\right\}}}{\cdots} \ldots\right| \\
& \leq \int_{\substack{\left\{t \in\left[\tau_{1}+1, \tau_{2}+1\right] \\
z\left(t, g_{s}\right) \in\left[z_{3}, z_{0}\right]\right\}}}\left|g_{s}\left(z\left(t, g_{s}\right)\right)\right| \frac{\dot{z}\left(t, g_{s}\right)}{K / 2 \tilde{\theta}} d t+\left(\tau_{2}-\tau_{1}\right) \max \left\{|g|_{C^{0}}, M_{2}\right\} \\
& \leq \frac{2 \tilde{\theta}}{K} g_{s}\left(z_{\max }\right) \int_{\left\{t \in\left[\tau_{1}+1, \tau_{2}+1\right]:\right.} \dot{z}\left(t, g_{s}\right) d t+\tilde{\theta} \cdot \max \{\ldots\} \\
& \left.z\left(t, g_{s}\right) \in\left[z_{3}, z_{0}\right]\right\} \\
& \left(\text { recall } 1+\theta_{+}<\tau_{1}<\tau_{2}<1+\theta_{+}+\tilde{\theta}\right) \\
& \leq \frac{2 \tilde{\theta}}{K} \frac{K+1}{\tilde{\theta}}\left(z_{0}-z_{3}\right)+\tilde{\theta} \cdot \max \{\ldots\} \\
& \leq\left[\frac{2(K+1)}{K}|g|_{C^{0}}+\max \left\{|g|_{C^{0}}, M_{2}\right\}\right] \tilde{\theta} .
\end{aligned}
$$

Putting together the estimates (4.35), (4.36) and (4.37), we get

$$
\begin{aligned}
& \left|z\left(3+\theta_{+}+\tilde{\theta}, g_{s}\right)-z\left(3+\theta_{+}, g_{s}\right)\right| \\
\leq & \left|\int_{2+\theta_{+}}^{\tau_{1}+1} g_{s}\left(z\left(t, g_{s}\right)\right)\right|+\left|\int_{\tau_{1}+1}^{\tau_{2}+1} \ldots\right|+\left|\int_{\tau_{2}+1}^{2+\theta_{+}+\tilde{\theta}} \ldots\right| \\
\leq & \tilde{\theta} M_{2}+\tilde{\theta}\left[\frac{2(K+1)}{K}|g|_{C^{0}}+\max \left\{|g|_{C^{0}}, M_{2}\right\}\right]+\tilde{\theta}|g|_{C^{0}} .
\end{aligned}
$$

Using (4.21), Proposition 3.4,a), and the definition of $\iota$, we can estimate the last term by

$$
\frac{k_{4} k_{1} k_{3} \delta}{64} \leq \frac{k_{4} k_{1} \delta_{1}}{8}=\frac{k_{4} \iota}{8} .
$$

Together with (4.34), we obtain (4.32).

Proof of (4.33): Using Taylor expansion of $\psi_{s}$ at 0 , and the properties $\theta_{+} \leq$ $\delta, \tilde{\theta} \leq \delta$, one gets

$$
\left|\psi_{s}(0)-\psi_{s}\left(-\theta_{+}-\tilde{\theta}-\delta\right)\right| \leq|\underbrace{\dot{\psi}_{s}(0)}_{=0}|+M_{2} \frac{\left(\theta_{+}+\tilde{\theta}+\delta\right)^{2}}{2} \leq \frac{9 M_{2}}{2} \delta^{2} .
$$

With (3.7)(vi) and, as before, the relation between $\delta$ and $\delta_{1}$ from Proposition 3.4,a), we can estimate the last term by

$$
\frac{k_{4} k_{1} k_{3} \delta}{32} \leq \frac{k_{4} k_{1} \delta_{1}}{4}=\frac{k_{4} \iota}{4} .
$$

Estimate (4.33) is proved. Estimate (4.30) now follows from (4.32) and (4.33). 


\section{Comments on the proof of Lemma 4.7.}

1) Recall that, from Proposition 3.4, the number $\delta$ is essentially a measure for the distance between the segments $\varphi$ and $y_{0}$. Recall also that $\delta_{1}$ and $\iota$ are essentially proportional to $\delta$. The error estimate (4.30) with $\iota$ on the right hand side is the key point of the above proof. It was obtained by estimating the left hand sides of (4.32) and (4.33) in terms of powers of $\delta$ which are larger than one-see (4.34) and the proof of (4.33). In comparison with the paper [L-WW2], this technical detail is new. It allows us to solve the problems which had been discussed in the comment following Proposition 3.4 of [L-WW2] by use of relatively simple nonlinearities. In the latter paper, a solution was found at the expense of a more complicated shape of the nonlinearities and the homoclinic solution.

2) Note that the values of $g_{s}$ in the vicinity of $z_{\max }$ have a 'large' effect on the solution $z\left(\cdot, g_{s}\right)$ when these solutions 'feel' the maximum of $g_{s}$ for the first time (namely, an increase from $z\left(2+\theta_{+}, g_{1}\right)$ to 0$)$. The same values of $g_{s}$ have only a 'small' effect when the solution crosses $z_{\max }$ for the second time, as is expressed in the estimate (4.37). The main point of that estimate is that when $z\left(\cdot, g_{s}\right)$ passes the interval $\left[z_{3}, z_{0}\right]$ (where $g_{s}$ has large values) for the second time, it passes at high 'speed' $\left(\dot{z}\left(t, g_{s}\right) \geq K / 2 \tilde{\theta}\right)$. To establish this property was the purpose of the two estimates from Lemma 4.5,c), and of the corresponding estimates in Proposition 4.2, (vi). A similar technique was used in [L-WW2].

\section{Providing transversality}

We see from Lemma 4.7 that the solutions $z\left(\cdot, g_{s}\right)$ reach the targets $\psi_{s}(s \in[0,1])$, up to an error of at most $k_{4} \iota / 2$. We could already use these nonlinearities to obtain a homoclinic solution, but we need to add a perturbation that makes sure that the homoclinic solution will be transversal. The perturbation must be such that it essentially keeps the accuracy by which the targets $\psi_{s}$ are approximated. The method to achieve this is as in [L-WW2]; we add a perturbation to the $g_{s}$ which has small $C^{0}$ - norm, but 'large' $C^{1}$-norm, so that it has significant influence on the solutions of the variational equations $\left(g_{s}, z\left(\cdot, g_{s}\right)\right)$, but little influence on the solutions $z\left(\cdot, g_{s}\right)$.

Recall condition $(\mathrm{T})$ from Theorem 2.9. We will actually prove a stronger property, namely, that segments of solutions $a \cdot \dot{z}+b \cdot w \quad\left((a, b) \in \mathbb{R}^{2} \backslash\{(0,0)\}\right)$ are all contained in the set $S$ at a fixed time which is independent of $a$ and $b$. (In fact, at time $t^{*}+1$.) Proving this stronger property requires a perturbation that acts, so to speak, violently on the solutions of the variational equations $\left(g_{s}, z\left(\cdot, g_{s}\right)\right)$. At present, however, we have no other method available.

Set $z_{\min }:=z\left(t_{\min }, g_{1}\right)$. We want to apply Propositions 2.3 and Propositions 2.4 from [L-WW2] (in the corresponding version for a minimum) to $z\left(\cdot, g_{1}\right)$ and $w\left(\cdot, g_{1}\right)$ at $t_{\min }$. We cite these results as 'Proposition 2.3*' and 'Proposition 2.4*' in the sequel. First, $z\left(\cdot, g_{1}\right)$ is $C^{2}$, since it is a solution of eq. $\left(g_{1}\right)$ on all of $\mathbb{R}$. For $s \in[0,1]$, we have

$$
\begin{aligned}
& \dot{z}\left(t_{\min }, g_{s}\right)=\dot{z}\left(t_{\min }, g_{1}\right)=0, \\
& \ddot{z}\left(t_{\min }, g_{s}\right)=\ddot{z}\left(t_{\min }, g_{1}\right)=g^{\prime}(0) \dot{z}\left(t_{0}, g_{1}\right)>0 .
\end{aligned}
$$

In the notation of Proposition $2.3^{*}$, we set $a:=2-\delta, b:=2, z:=z\left(\cdot, g_{1}\right) \mid[a, b]$. Application of Proposition $2.3^{*}$,a) yields numbers $s_{1}, s_{2} \in[2-\delta, 2]$ and $d>0$ such 
that for every $\rho \in(0, d]$ there exist unique numbers $\sigma_{1}(\rho), \sigma_{2}(\rho)$ with the properties

$$
\begin{gathered}
s_{1} \leq \sigma_{1}(\rho)<t_{\min }<\sigma_{2}(\rho) \leq s_{2} \\
z(t) \leq z_{\min }+\rho \text { for } t \in\left[\sigma_{1}(\rho), \sigma_{2}(\rho)\right], \\
z(t)>z_{\min }+\rho \text { for } t \in\left[s_{1}, \sigma_{1}(\rho)\right) \cup\left(\sigma_{2}(\rho), s_{2}\right] .
\end{gathered}
$$

It is clear that these properties hold also for every $\tilde{d} \in(0, d]$. We can therefore choose $d$ such that, in addition,

$$
z_{\min }+d \leq z_{4}
$$

We prepare an application of Proposition $\left.2.3^{*}, \mathrm{~b}\right)$. In the notation of that proposition, we set

$$
\begin{aligned}
\gamma & :=\frac{1}{2} \min \left\{g_{s}\left(z_{\min }-1\right): s \in[0,1]\right\}>0 \\
\Delta & :=k_{4} \iota / 2 \\
W_{0} & :=\sup \left\{\left|w\left(t, g_{s}\right)\right|: t \in\left[t^{*}-1, t^{*}\right], s \in[0,1]\right\} ; \\
\zeta_{0} & :=-\max \left\{g^{\prime}(x): x \in\left[-y(0)-\delta_{1}, y(0)+\delta_{1}\right]\right\} \cdot \gamma>0 .
\end{aligned}
$$

Further, we choose a number $W_{1}>W_{0}$ such that

$$
|g|_{C^{1}} W_{0} \leq W_{1} / 2 \text { and } \frac{\left(|g|_{C^{1}}\right)^{2} W_{0}}{\zeta_{0}} \leq W_{1} / 2
$$

and we set

$$
W:=-3 W_{1}<0 .
$$

We know from Proposition 4.3,f) that $w\left(t_{\min }, g_{1}\right)<0$. We apply Proposition $\left.2.3^{*}, \mathrm{~b}\right)$ with $W, \gamma, \Delta$ and $d, s_{1}, s_{2}$ from above, and with $w\left(\cdot, g_{1}\right)$. We obtain a number $\rho \in(0, d]$ with

$$
\gamma\left(\sigma_{2}(\rho)-\sigma_{1}(\rho)\right) \leq \delta
$$

and a function $h \in B C^{1}(\mathbb{R}, \mathbb{R})$ with the following properties.

(i) $h(x)=0$ for $x \geq z_{\min }+\rho$;

(ii) $h(x)=h\left(z_{\min }\right)$ for $x \leq z_{\min }$;

(iii) $|h(x)| \leq \gamma$ for $x \in \mathbb{R}$;

(iv) $\operatorname{sign} h(x)=-\operatorname{sign}\left(w\left(t_{\min }\right), g_{1}\right) \cdot \operatorname{sign}\left(-3 W_{1}\right)=-1$ for $x \in\left(-\infty, z_{\min }+\rho\right)$;

(v) $\operatorname{sign} h^{\prime}\left(z\left(t, g_{1}\right)\right) w\left(t, g_{1}\right)=\operatorname{sign}\left(-3 W_{1}\right)=-1$ for $t \in\left(\sigma_{1}(\rho), \sigma_{2}(\rho)\right) \backslash\left\{t_{\min }\right\}$, so $h^{\prime}\left(z\left(t, g_{1}\right)\right)>0$ and $w\left(t, g_{1}\right)<0$ for these $t$;

(vi) $\int_{\sigma_{1}(\rho)}^{\sigma_{2}(\rho)} h^{\prime}\left(z\left(t, g_{1}\right)\right) w\left(t, g_{1}\right) d t=-3 W_{1}$.

Note that the minus sign in property (iv) is part of the analogous reformulation of Proposition $\left.2.3^{*}, \mathrm{~b}\right)$ for the case of a minimum, since, in this case, sign $h(x)=$ $-\operatorname{sign} h^{\prime}(x)$ for $x \in\left(z_{\min }, z_{\min }+\rho\right)$.

Next, we apply the analogue of Proposition $2.4^{*}$ for the case of a minimum to the functions $g_{s}$ and to $z\left(\cdot, g_{s}\right)$, for all $s \in[0,1]$. Let $s \in[0,1]$. In the notation of Proposition $2.4^{*}$, we set $t_{0}:=0, z:=z\left(\cdot,\left.g_{s}\right|_{[-1, \infty)}, w:=w\left(\cdot, g_{s}\right)\right.$, and we take $\gamma, W$ and $\Delta$ as above. The assumptions of Proposition $2.4^{*}$ are satisfied. 
Note that $z\left(\cdot, g_{s}\right)=z\left(\cdot, g_{1}\right), \quad w\left(\cdot, g_{s}\right)=w\left(\cdot, g_{1}\right)$ on $[2-\delta, 2]$, and that the function $h$ from above does not depend on $s$. Note also that $g_{s}$ and $g_{s}+h$ are both modifications of $g_{1}$ on $\left(-\infty, z\left(1+\theta_{+}, g_{1}\right)\right] \subset(-\infty,-y(0)-1]$, and that $z\left(t, g_{1}\right) \geq$ $z\left(1+\theta_{+}, g_{1}\right)$ for $t \in\left(-\infty, 1+\theta_{+}\right]$(see Proposition 3.4,d), and Proposition 4.3,d)). It follows that

$$
\begin{aligned}
z\left(\cdot, g_{s}\right) & =z\left(\cdot, g_{s}+h\right)=z\left(\cdot, g_{1}\right) \text { on }\left(-\infty, 2+\theta_{+}\right], \text {and } \\
w\left(\cdot, g_{s}\right) & =w\left(\cdot, g_{s}+h\right)=w\left(\cdot, g_{1}\right) \text { on }\left[-1,2+\theta_{+}\right] .
\end{aligned}
$$

From Proposition $2.4^{*}$, together with 5.2 , we obtain the following properties for the nonlinearities $g_{s}+h$ and the solutions $z\left(\cdot, g_{s}+h\right)$, respectively $w\left(\cdot, g_{s}+h\right)$.

$$
\begin{aligned}
z\left(t, g_{s}+h\right) & =z\left(t, g_{s}\right) \text { for } t \in\left(-\infty, \sigma_{1}(\rho)+1\right), \text { and } \\
w\left(t, g_{s}+h\right) & =w\left(t, g_{s}\right) \text { for } t \in\left[-1, \sigma_{1}(\rho)+1\right] ;
\end{aligned}
$$

(ii) $\left|z\left(t, g_{s}+h\right)-z\left(t, g_{s}\right)\right| \leq \Delta$ for $t \in\left[\sigma_{1}(\rho)+1, \sigma_{2}(\rho)+1\right]$;

(iii) $w\left(\sigma_{2}(\rho)+1, g_{s}+h\right)=w\left(\sigma_{2}(\rho)+1, g_{s}\right)-3 W_{1}$;

(iv) $\dot{z}\left(t, g_{s}+h\right)=\dot{z}\left(t, g_{s}\right)$ and $\dot{w}\left(t, g_{s}+h\right)=\dot{w}\left(t, g_{s}\right)$ for $t \in\left[\sigma_{2}(\rho)+1, \sigma_{1}(\rho)+2\right]$.

It follows from (5.3), (ii) and (5.3), (iv) that we have

$$
\left|z\left(t, g_{s}+h\right)-z\left(t, g_{s}\right)\right| \leq \Delta \text { for } t \in\left[\sigma_{1}(\rho)+1, \sigma_{1}(\rho)+2\right] .
$$

The functions $g_{s}+h$ do not satisfy $(\mathrm{NF})$, and their derivatives may have more than one zero. We modify these functions to new nonlinearities $f_{s}$ such that the above properties are preserved, and that $f_{s}$ satisfies $(\mathrm{NF})$ and $f_{s}^{\prime}$ has only one zero. Note that (5.1),(iii) and the choice of $\gamma$ imply $\left(g_{s}+h\right)\left(z_{\min }-1\right)>0$ for $s \in[0,1]$.

For $s \in[0,1]$, set $\lambda_{s}:=\frac{\left(g_{s}+h\right)^{\prime}\left(z_{\min }-1\right)}{\left(g_{s}+h\right)\left(z_{\min }-1\right)}$, and define $f_{s}$ by

$$
f_{s}(x):=\left\{\begin{array}{r}
\left(g_{s}+h\right)(x) \text { for } x>z_{\min }-1, \\
\left(g_{s}+h\right)\left(z_{\min }-1\right) \cdot \frac{e^{\lambda_{s}\left(x-\left(z_{\min }-1\right)\right)}}{1+\left[x-\left(z_{\min }-1\right)\right]^{2}} \text { for } x \leq z_{\min }-1 .
\end{array}\right.
$$

Proposition 5.1. For $s \in[0,1]$, the function $f_{s}$ is in $B C^{1}(\mathbb{R}, \mathbb{R})$, satisfies (NF), and $f_{s}^{\prime}>0$ on $\left(-\infty, z_{\max }\right), \quad f_{s}^{\prime}<0$ on $\left(z_{\max }, \infty\right)$.

The map $[0,1] \ni s \mapsto f_{s} \in\left(B C^{0}(\mathbb{R}, \mathbb{R}),||_{C^{0}}\right)$ is continuous. Further,

$$
\begin{gathered}
z\left(t, f_{s}\right)=z\left(t, g_{s}+h\right) \text { for } t \in\left(-\infty, t^{*}+1\right], \\
w\left(t, f_{s}\right)=w\left(t, g_{s}+h\right) \text { for } t \in\left[-1, t^{*}+1\right] .
\end{gathered}
$$

Proof. Let $s \in[0,1]$. First, $\lim _{x \longrightarrow z_{\min }-1-} f_{s}(x)=\left(g_{s}+h\right)\left(z_{\min }-1\right)$ and

$$
\begin{aligned}
\lim _{x \longrightarrow z_{\min }-1-} f_{s}^{\prime}(x) & =\lambda_{s} f_{s}\left(z_{\min }-1\right)=\left(g_{s}+h\right)^{\prime}\left(z_{\min }-1\right) \\
& =\lim _{x \longrightarrow z_{\min }-1+} f_{s}^{\prime}(x) .
\end{aligned}
$$

The property $f_{s} \in B C^{1}(\mathbb{R}, \mathbb{R})$ now follows from $g_{s}+h \in B C^{1}(\mathbb{R}, \mathbb{R})$ and the definition of $f_{s}$. Note that $f_{s}^{\prime}(x)>0$ for $x \leq z_{\text {min }}-1$. From 5.1(i),(ii) and (v), we have $h^{\prime} \geq 0$. Since $g_{s}^{\prime}>0$ on $\left(-\infty, z_{\max }\right)$, we have $f_{s}^{\prime}>0$ on $\left(-\infty, z_{\max }\right)$. We have $f_{s}(x)=g_{s}(x)$ for $x \geq z_{\text {min }}+d$ since $h(x)=0$ for these $x$. Hence, $f_{s}^{\prime}=g_{s}^{\prime}<0$ on $\left(z_{\max }, \infty\right)$. In order to prove $(\mathrm{NF})$ for $f_{s}$, it suffices to show that $f_{s}>0$ on $\left(-\infty, z_{\min }+d\right]$. It is obvious that $f_{s}(x)>0$ for $x \in\left(-\infty, z_{\min }-1\right]$. For 
$x \in\left(z_{\min }-1, z_{\min }+d\right]$, we have $g_{s}(x)>g_{s}\left(z_{\min }-1\right)$, and, recalling the definition of $\gamma$,

$$
\begin{aligned}
f_{s}(x)=\left(g_{s}+h\right)(x) & >g_{s}\left(z_{\min }-1\right)+h(x) \\
& \geq 2 \gamma-\gamma=\gamma>0 .
\end{aligned}
$$

It follows from Lemma 4.5 that the map $[0,1] \ni s \mapsto g_{s}+h \in\left(B C^{0}(\mathbb{R}, \mathbb{R}),||_{C^{0}}\right)$ is continuous. The definition of the functions $g_{s}$ on $\left(-\infty, z_{4}\right]$, together with the property $z_{\min }+d \leq z_{4}$ and with continuity of the map

$$
[0,1] \ni s \mapsto \psi_{s} \in\left(C^{2}([-1,0], \mathbb{R}),||_{C^{2}}\right)
$$

implies that the maps $[0,1] \ni s \mapsto \lambda_{s} \in \mathbb{R}$ and

$$
\left.\left.[0,1] \ni s \mapsto f_{s}\right|_{\left(-\infty, z_{\min }-1\right]} \in B C^{0}\left(\left(-\infty, z_{\min }-1\right], \mathbb{R}\right),||_{C^{0}}\right)
$$

are continuous. With the definition of $f_{s}$, the continuity assertion of Proposition 5.1 follows.

Proof of (5.5): Using 5.3,(i) and $\sigma_{1}(\rho)>2-\delta$, we see that $z\left(t, g_{s}+h\right)=z\left(t, g_{s}\right)$ for $t \in(-\infty, 3-\delta]$. With Lemma 4.7,b) and Proposition 4.3,d), and with the last property from Proposition $3.4, \mathrm{~d})$, we obtain

$$
\begin{aligned}
& z\left(t, g_{s}+h\right)=z\left(t, g_{s}\right) \geq z_{\min } \text { for } t \in(-\infty, 3-\delta], \text { and } \\
& z\left(t, g_{s}+h\right)=z\left(t, g_{s}\right)<0 \text { for } t \in\left[2-\delta, 2+\theta_{+}+\tilde{\theta}\right) .
\end{aligned}
$$

It follows from (5.6) that $\left(g_{s}+h\right)(x)>0$ for $x \in\left(z_{\min }, 0\right)$ (although $g_{s}+h$ does not satisfy (NF) for all $x)$. Hence we get that $\dot{z}\left(t, g_{s}+h\right)>0$ for $t \in\left[3-\delta, 3+\theta_{+}+\tilde{\theta}\right]$, so that together we obtain $z\left(t, g_{s}+h\right) \geq z_{\min }$ for $t \in\left(-\infty, t^{*}\right]$. Property (5.5) is now a consequence of $f_{s}=g_{s}+h$ on $\left[z_{\min }, \infty\right)$.

In the next lemma, we prove that the segments $z\left(\cdot, f_{s}\right)_{t^{*}}$ still approximate the targets $\psi_{s}(s \in[0,1])$, and that, in addition, a transversality property holds uniformly with respect to $s \in[0,1]$. This uniformity is necessary for our method of proof: We will find an $s^{*} \in(0,1)$ with the property that the orbit of $z\left(\cdot, f_{s^{*}}\right)$ is homoclinic to $\mathcal{O}$ by an intermediate value argument - therefore we have no information about where in $[0,1]$ this $s^{*}$ lies.

Recall the set $S$ of segments with at most one sign change.

Lemma 5.2. Let $s \in[0,1]$. The functions $z\left(\cdot, f_{s}\right)$ and $w\left(\cdot, f_{s}\right)$ have the following properties:

a) $z\left(\cdot, f_{s}\right)_{t^{*}} \in H,\left|z\left(\cdot, f_{s}\right)_{t^{*}}-\psi_{s}\right| \leq k_{4} \iota$.

b) If the condition

$$
\left|z\left(t, f_{s}\right)\right|<y(0)+\delta_{1} \text { for all } t \in\left[t^{*}-1, t^{*}+1\right]
$$

holds, then we have

$$
\forall(a, b) \in \mathbb{R}^{2} \backslash\{(0,0)\}:\left[a \dot{z}\left(\cdot, f_{s}\right)+b \cdot w\left(\cdot, f_{s}\right)\right]_{t^{*}+1} \in S .
$$

Proof. Let $s \in[0,1]$.

Proof of a): Recall property (5.5). Together with (5.3),(i), we obtain

$$
z\left(t, f_{s}\right)=z\left(t, g_{s}\right) \text { for } t \in\left(-\infty, \sigma_{1}(\rho)+1\right]
$$

In particular, Lemma $4.7, \mathrm{c})$ shows that $z\left(t^{*}-1, f_{s}\right)=0$, so $z\left(\cdot, f_{s}\right)_{t^{*}} \in H$.

Combining (5.5) and (5.4), and observing $\sigma_{1}(\rho)+2 \leq 2+2 \leq t^{*}+1$, we get

$$
\left|z\left(t, f_{s}\right)-z\left(t, g_{s}\right)\right| \leq \Delta \text { for } t \in\left[\sigma_{1}(\rho)+1, \sigma_{1}(\rho)+2\right] .
$$


Since $\sigma_{1}(\rho)+2>2-\delta+2>3+\theta_{+}+\tilde{\theta}=t^{*}$, we conclude from Lemma 4.7,c), from (5.8) and (5.9), and from the choice of $\Delta$ that

$$
\begin{aligned}
\left|z\left(\cdot, f_{s}\right)_{t^{*}}-\psi_{s}\right| & \leq\left|z\left(\cdot, f_{s}\right)_{t^{*}}-z\left(\cdot, g_{s}\right)_{t^{*}}\right|+\left|z\left(\cdot, g_{s}\right)_{t^{*}}-\psi_{s}\right| \\
& \leq \Delta+k_{4} \iota / 2=k_{4} \iota .
\end{aligned}
$$

Proof of b): Assume condition (5.7). It follows from (5.3),(iii), from $\sigma_{2}(\rho)+1 \in$ $(3-\delta, 3) \subset\left[t^{*}-1, t^{*}\right]$, from the definition of $W_{0}$ and the choice of $W_{1}$ that we have $w\left(\sigma_{2}(\rho)+1, g_{s}+h\right) \leq W_{0}-3 W_{1} \leq-2 W_{1}$. We set

$$
\bar{t}(s):=\min \left\{t \in\left[\sigma_{1}(\rho)+1, \sigma_{2}(\rho)+1\right]: w\left(t, g_{s}+h\right) \leq 0\right\} ;
$$

then $\bar{t}(s)<\sigma_{2}(\rho)+1$. Note also that

$$
3-\delta<\sigma_{1}(\rho)+1 \leq \bar{t}(s)<\sigma_{2}(\rho)+1<3 .
$$

(5.11) Claim: $w\left(\cdot, f_{s}\right)$ has the following properties:

(i) $\dot{w}\left(t, f_{s}\right)<0$ for $t \in\left[\sigma_{1}(\rho)+1, \sigma_{2}(\rho)+1\right]$;

(ii) $\left|w\left(t, f_{s}\right)\right| \leq W_{0}$ for $t \in\left[t^{*}-1, \bar{t}(s)\right]$;

(iii) $\left|\dot{w}\left(t, f_{s}\right)\right| \leq|g|_{C^{1}} W_{0}$ for $t \in\left[t^{*}, \bar{t}(s)+1\right]$;

$$
\begin{aligned}
\left|w\left(t, f_{s}\right)\right| & \leq 4 W_{1} \text { for } t \in\left[\sigma_{1}(\rho)+1, t^{*}\right], \text { and } \\
w\left(t, f_{s}\right) & \leq-2 W_{1} \text { for } t \in\left[\sigma_{2}(\rho)+1, t^{*}\right] ;
\end{aligned}
$$

(v) $\left|\dot{w}\left(t, f_{s}\right)\right| \leq 4|g|_{C^{1}} W_{1}$ for $t \in\left[\bar{t}(s)+1, t^{*}+1\right]$.

(vi) $w\left(t, f_{s}\right) \leq-W_{1}$ for $t \in\left[t^{*}, t^{*}+1\right]$;

(vii) $\dot{w}\left(t, f_{s}\right)>0$ for $t \in\left(\bar{t}(s)+1, t^{*}+1\right]$.

Proof. In view of (5.5), it suffices to prove the corresponding assertions for the function $w\left(\cdot, g_{s}+h\right)$.

Ad (i): For $t \in\left[\sigma_{1}(\rho)+1, \sigma_{2}(\rho)+1\right]$, we have $z\left(t-1, g_{1}\right) \leq z_{\min }+d \leq z_{4}<z_{3}<$ $z_{\max }$. Hence we obtain from (5.2), from (5.1),(v), and from $g_{s}^{\prime}>0$ on $\left(-\infty, z_{\max }\right)$ that

$$
\begin{aligned}
\dot{w}\left(t, g_{s}+h\right) & =\left(g_{s}+h\right)^{\prime}\left(z\left(t-1, g_{s}+h\right)\right) w\left(t-1, g_{s}+h\right) \\
& =\left(g_{s}+h\right)^{\prime}\left(z\left(t-1, g_{1}\right)\right) w\left(t-1, g_{1}\right)<0 .
\end{aligned}
$$

Ad (ii): For $t \in\left[t^{*}-1, \sigma_{1}(\rho)+1\right]$, we have from the definition of $W_{0}$ and from (5.3),(i) that

$$
\left|w\left(t, g_{s}+h\right)\right|=\left|w\left(t, g_{s}\right)\right| \leq W_{0} .
$$

Let $t \in\left[\sigma_{1}(\rho)+1, \bar{t}(s)\right]$. If $\bar{t}(s)=\sigma_{1}(\rho)+1$ then

$$
\left|w\left(t, g_{s}+h\right)\right|=\left|w\left(\sigma_{1}(\rho)+1, g_{s}\right)\right| \leq W_{0},
$$

and otherwise one has

$$
w\left(t, g_{s}+h\right) \in\left[0, w\left(\sigma_{1}(\rho)+1, g_{s}\right)\right] \subset\left[0, W_{0}\right] .
$$

Ad (iii): We have $g_{s}+h=g$ on $\left[-y(0)-\delta_{1}, y(0)+\delta_{1}\right]$. From condition (5.7) and the estimate (5.11),(ii), we obtain that for $t \in\left[t^{*}, \bar{t}(s)+1\right]$

$$
\left|\dot{w}\left(t, g_{s}+h\right)\right|=\left|g^{\prime}\left(z\left(t-1, f_{s}\right)\right) w\left(t-1, g_{s}+h\right)\right| \leq|g|_{C^{1}} W_{0} .
$$


Ad (iv): For $t \in\left[\sigma_{1}(\rho)+1, \sigma_{2}(\rho)+1\right]$, properties (5.3),(i), (5.11),(i) and (5.3),(iii) show that

$$
\begin{aligned}
w\left(t, g_{s}+h\right) & \in\left[w\left(\sigma_{2}(\rho)+1, g_{s}+h\right), w\left(\sigma_{1}(\rho)+1, g_{s}+h\right)\right] \\
& =\left[w\left(\sigma_{2}(\rho)+1, g_{s}\right)-3 W_{1}, w\left(\sigma_{1}(\rho)+1, g_{s}\right)\right] .
\end{aligned}
$$

Using the definition of $W_{0}$ and $W_{1}$, one sees that

$$
w\left(t, g_{s}+h\right) \in\left[-W_{0}-3 W_{1}, W_{0}\right] \subset\left[-4 W_{1}, 4 W_{1}\right] .
$$

For $t \in\left[\sigma_{2}(\rho)+1, t^{*}\right]$ we obtain from (5.3),(iv) and from $\sigma_{1}(\rho)+2>4-\delta>t^{*}$ that

$$
\begin{aligned}
w\left(t, g_{s}+h\right) & =w\left(t, g_{s}\right)-3 W_{1} \in\left[-W_{0}-3 W_{1}, W_{0}-3 W_{1}\right] \\
& \subset\left[-4 W_{1},-2 W_{1}\right] .
\end{aligned}
$$

Combining (5.12) and (5.13) yields property (iv).

Ad (v): From (5.10), we have $\left[\bar{t}(s)+1, t^{*}+1\right] \subset\left[\sigma_{1}(\rho)+2, t^{*}+1\right]$. Using condition (5.7) together with (5.5) and the first estimate in (5.11),(iv), one gets that for $t \in\left[\bar{t}(s)+1, t^{*}+1\right]$,

$$
\left|\dot{w}\left(t, g_{s}+h\right)\right|=\left|g^{\prime}\left(z\left(t-1, f_{s}\right)\right) w\left(t-1, g_{s}+h\right)\right| \leq|g|_{C^{1}} 4 W_{1} .
$$

Ad (vi): For $t \in\left[t^{*}, t^{*}+1\right]$, we obtain from properties (iv), (iii) and (v) that

$$
w\left(t, g_{s}+h\right) \leq-2 W_{1}+\left(\bar{t}(s)+1-t^{*}\right)|g|_{C^{1}} W_{0}+\left(t^{*}+1-(\bar{t}(s)+1)\right) 4|g|_{C^{1}} W_{1} .
$$

Using (5.10), we see that

$$
w\left(t, g_{s}+h\right) \leq-2 W_{1}+|g|_{C^{1}} W_{0}+\left(\theta_{+}+\tilde{\theta}+\delta\right) 4|g|_{C^{1}} W_{1} .
$$

With the choice of $\theta_{+}, \tilde{\theta}, \delta$ (see (3.7),(iii)) and of $W_{1}$, it follows that

$$
\begin{aligned}
w\left(t, g_{s}+h\right) & \leq-2 W_{1}+\frac{1}{2} W_{1}+12 \delta|g|_{C^{1}} W_{1} \\
& \leq-2 W_{1}+\frac{1}{2} W_{1}+\frac{1}{2} W_{1}=-W_{1} .
\end{aligned}
$$

Ad (vii): Combining (5.10), the definition of $\bar{t}(s)$, and (i) with the second property from (iv), we get that $w\left(t, g_{s}+h\right)<0$ for $t \in\left(\bar{t}(s), t^{*}\right]$. For these $t$, condition (5.7) together with (5.5) shows that $\left(g_{s}+h\right)^{\prime}\left(z\left(t, g_{s}+h\right)\right)=g^{\prime}\left(z\left(t, f_{s}\right)\right)<0$. The assertion now follows from the variational equation $\left(g_{s}+h, z\left(\cdot, g_{s}+h\right)\right)$.

(Claim (5.11) is proved.)

(5.14) Claim:

(i) $z\left(\cdot, f_{s}\right)$ is $C^{2}$ on $\left[t^{*}, t^{*}+1\right]$;

(ii) $\dot{z}\left(t^{*}, f_{s}\right)=0, \dot{z}\left(t, f_{s}\right) \in\left[-|g|_{C^{1}}, 0\right)$ for $t \in\left(t^{*}, t^{*}+1\right]$;

(iii) $\ddot{z}\left(t, f_{s}\right) \leq 0$ for $t \in\left[t^{*}, t^{*}+1\right]$;

(iv) $\ddot{z}\left(t, f_{s}\right) \leq-\zeta_{0}$ for $t \in\left[t^{*}, \bar{t}(s)+1\right]$.

Proof. Property (i) is a consequence of eq. $\left(f_{s}\right)$ and of $t^{*}>1$.

Ad (ii): From assertion a) of the lemma, we know $z\left(t^{*}-1, f_{s}\right)=0$, so $\dot{z}\left(t^{*}, f_{s}\right)=$ 0. One sees from (5.5), (5.3),(i), Lemma 4.7,a) and Proposition 4.3,d) that

$$
z\left(t, f_{s}\right)=z\left(t, g_{s}+h\right)=z\left(t, g_{s}\right)<0 \text { for } t \in\left(t_{0}, t^{*}-1\right),
$$

and this interval contains $\left[t^{*}-2, t^{*}-1\right)$. Now (NF) implies that

$$
\dot{z}\left(\cdot, f_{s}\right)>0 \text { on }\left[t^{*}-1, t^{*}\right), \quad \text { and } \quad z\left(\cdot, f_{s}\right)>0 \text { on }\left(t^{*}-1, t^{*}\right] .
$$


Using (NF) again, we infer $\dot{z}\left(\cdot, f_{s}\right)<0$ on $\left(t^{*}, t^{*}+1\right]$. Condition (5.7) and the property $f_{s}=g$ on $\left[-y(0)-\delta_{1}, y(0)+\delta_{1}\right]$ imply that $\dot{z}\left(t, f_{s}\right) \geq-|g|_{C^{1}}$ on this interval.

Ad (iii): For $t \in\left[t^{*}, t^{*}+1\right],(5.15)$ and (5.7) imply

$$
\ddot{z}\left(t, f_{s}\right)=\underbrace{g^{\prime}\left(z\left(t-1, f_{s}\right)\right)}_{<0} \cdot \underbrace{\dot{z}\left(t-1, f_{s}\right)}_{\geq 0} \leq 0 .
$$

Ad (iv): Let $t \in\left[t^{*}, \bar{t}(s)+1\right] \subset\left[t^{*}, 4\right] \subset\left[t^{*}, t^{*}+1\right]$. From (5.7), we have

$$
g^{\prime}\left(z\left(t-1, f_{s}\right)\right) \leq \max \left\{g^{\prime}(x): x \in\left[-y(0)-\delta_{1}, y(0)+\delta_{1}\right]\right\}<0 .
$$

Further, $t-2 \in\left[t^{*}-2, \bar{t}(s)-1\right] \subset\left[t^{*}-2,2\right]=\left[1+\theta_{+}+\tilde{\theta}, 2\right]$. From (5.5), (5.3),(i), and Lemma 4.7 ,a), we have $z\left(t-2, f_{s}\right)=z\left(t-2, g_{1}\right) \in\left[z_{\min }, z_{3}\right]$. Using the definition of $f_{s}$, the monotonicity properties of $f_{s}$ from Proposition 5.1, and (5.6), one gets

$$
\dot{z}\left(t-1, f_{s}\right)=f_{s}\left(z\left(t-2, f_{s}\right)\right) \geq f_{s}\left(z_{\min }\right) \geq \gamma>0 .
$$

Now (5.16) shows that

$$
\ddot{z}\left(t, f_{s}\right) \leq \max \left\{g^{\prime}(x): x \in\left[-y(0)-\delta_{1}, y(0)+\delta_{1}\right]\right\} \cdot \gamma=-\zeta_{0} .
$$

(Claim (5.14) is proved.)

We are now ready for the proof of assertion b) of Lemma 5.2. Let $(a, b) \in$ $\mathbb{R}^{2} \backslash\{(0,0)\}$, and note that the set $S \subset C$ is invariant under multiplication with nonzero reals.

In case $b=0$, we have $a \neq 0$, and assertion b) is equivalent to $\left[\dot{z}\left(\cdot, f_{s}\right)\right]_{t^{*}+1} \in S$, which follows from (5.14)(ii).

In case $b \neq 0$, we set $c:=a / b$, and the assertion is equivalent to

$$
\left[c \dot{z}\left(\cdot, f_{s}\right)+w\left(\cdot, f_{s}\right)\right]_{t^{*}+1} \in S .
$$

First case: $c \geq \frac{-|g|_{C^{1}} W_{0}}{\zeta_{0}}$. Then we have from (5.11),(vi), from (5.14),(ii), and from the choice of $W_{1}$ that for $t \in\left[t^{*}, t^{*}+1\right]$,

$$
c \dot{z}\left(t, f_{s}\right)+w\left(t, f_{s}\right) \leq \frac{|g|_{C^{1}} W_{0}}{\zeta_{0}}|g|_{C^{1}}-W_{1} \leq-W_{1} / 2<0,
$$

from which (5.17) follows.

Second case: $c<\frac{-|g|_{C^{1}} W_{0}}{\zeta_{0}}$. Then, for $t \in\left[t^{*}, \bar{t}(s)+1\right]$, properties (5.11),(iii) and (5.14),(iv) show that

$$
c \ddot{z}\left(t, f_{s}\right)+\dot{w}\left(t, f_{s}\right)>\frac{-|g|_{C^{1}} W_{0}}{\zeta_{0}}\left(-\zeta_{0}\right)-|g|_{C^{1}} W_{0}=0 .
$$

Further, for $t \in\left(\bar{t}(s)+1, t^{*}+1\right]$, properties (5.11),(vii) and (5.14),(iii) show

$$
c \ddot{z}\left(t, f_{s}\right)+\dot{w}\left(t, f_{s}\right) \geq \dot{w}\left(t, f_{s}\right)>0 .
$$

Together, we obtain that the segment $\left[c \dot{z}\left(\cdot, f_{s}\right)+w\left(\cdot, f_{s}\right)\right]_{t^{*}+1}$ is strictly increasing, and therefore (5.17) holds. Lemma 5.2 is proved.

Remark 5.3. The proof of Lemma 5.2 is similar to the proof of Lemma 3.8 from [L-WW2]. See the comment preceding that proof for an explanation of the basic principle. 


\section{TRANSVERSALLY HOMOCLINIC SOLUTIONS}

We are now ready to conclude our construction of a nonlinearity with only one extremum that generates a semiflow with transversally homoclinic orbits. The missing step is an intermediate value argument.

Theorem 6.1. $\quad$ a) There exists $s^{*} \in[0,1]$ such that the solution $z\left(\cdot, f_{s^{*}}\right)$ has a phase curve homoclinic to the orbit $\mathcal{O}$ of the periodic solution $y$ of eq. $\left(f_{s^{*}}\right)$.

b) The nonlinearity $f_{s^{*}}$ satisfies $(\mathrm{NF})$, and

$$
f_{s^{*}}^{\prime}>0 \text { on }\left(-\infty, z_{\max }\right), \quad f_{s^{*}}^{\prime}<0 \text { on }\left(z_{\max }, \infty\right) .
$$

c) The assumptions of Theorem 2.9 are satisfied by eq. $\left(f_{s^{*}}\right)$, the periodic solution $y$, by $\varphi=z\left(\cdot, f_{s^{*}}\right)_{0}$ and by $w\left(\cdot, f_{s^{*}}\right)$. From Theorems 2.9 and 2.7, we therefore obtain a Poincaré map with the property that the maximal invariant set of $P$ is hyperbolic. The action of $P$ on the trajectories in this set is conjugate to a symbol shift. The solutions of eq. $\left(f_{s^{*}}\right)$ corresponding to these trajectories are slowly oscillating.

Proof. Set $\Omega_{s}:=\left[z\left(\cdot, f_{s}\right)\right]_{t^{*}}$ for $s \in[0,1]$. Recall that $\psi_{0}=\psi_{-}, \psi_{1}=\psi_{+}$. From Lemma 5.2, a), and Proposition 3.3,(iii), we have $\Omega_{s} \in N_{\iota} \subset H$, and $\mathcal{H}\left(\Omega_{1}\right)>$ $0>\mathcal{H}\left(\Omega_{0}\right)$. Continuous dependence on initial data, together with continuity of the map $[0,1] \ni s \mapsto f_{s} \in\left(B C^{0}(\mathbb{R}, \mathbb{R}),||_{C^{0}}\right)$ and continuity of $\mathcal{H}$ implies that the map $[0,1] \ni s \mapsto \mathcal{H}\left(\Omega_{s}\right) \in C$ is continuous. It follows from the Intermediate Value Theorem that there exists $s^{*} \in[0,1]$ with $\mathcal{H}\left(\Omega_{s^{*}}\right)=0$. Now property (3.1), applied to $\iota \in(0, \bar{\delta}]$, shows that $\Omega_{s^{*}} \in W^{s}\left(y_{0}, P, N_{\iota}\right)$. Remark 4.1 now yields that $z\left(\cdot, f_{s^{*}}\right)$ is a solution of eq. $\left(f_{s^{*}}\right)$ with phase curve homoclinic to the orbit of $y$. Assertion a) is proved, and assertion b) is clear from Proposition 5.1.

Proof of assertion c): Set $\Omega^{*}:=\Omega_{s^{*}} \in W^{s}\left(y_{0}, P, N_{\iota}\right)$. Recall that $\iota=k_{1} \delta_{1}$ and, from Proposition 3.4,a), that $\delta_{1} \leq \bar{\delta}$. We see from Proposition 3.1,b) that the solution $x^{\Omega^{*}}$ of the unmodified eq. $(g)$ with $x_{0}^{\Omega^{*}}=\Omega^{*}$ satisfies $\forall t \geq 0: x_{t}^{\Omega^{*}} \in$ $\mathcal{O}+C\left(\delta_{1}\right)$, so that we have

$$
x^{\Omega^{*}}(t) \in\left(-y(0)-\delta_{1}, y(0)+\delta_{1}\right) \text { for all } t \in[-1, \infty) .
$$

Since $f_{s}^{*}=g$ on $\left(-y(0)-\delta_{1}, y(0)+\delta_{1}\right)$, we obtain

$$
z\left(t^{*}+t, f_{s^{*}}\right)=x^{\Omega^{*}}(t) \in\left(-y(0)-\delta_{1}, y(0)+\delta_{1}\right) \text { for all } t \in[-1, \infty) .
$$

In particular, condition (5.7) of Lemma 5.2 is satisfied. Moreover,

$$
f_{s^{*}}^{\prime}\left(z\left(t, f_{s^{*}}\right)\right)=g^{\prime}\left(z\left(t, f_{s^{*}}\right)\right)<0 \text { for } t \geq t^{*}-1,
$$

so that the variational equation $\left(f_{s^{*}}, z\left(\cdot, f_{s^{*}}\right)\right)$ has a negative coefficient for $t \geq$ $t^{*}-1$. It follows from this property, together with Lemma 5.2,b) and Proposition 4.6 of [L-WW1] that the function $a \cdot \dot{z}\left(\cdot, f_{s^{*}}\right)+b \cdot w\left(\cdot, f_{s^{*}}\right)$ is eventually slowly oscillating for all $(a, b) \in \mathbb{R}^{2} \backslash\{(0,0)\}$. Hence condition (T) of Theorem 2.9 holds, and the conclusions follow.

\section{REPORT ON NUMERICAL EXPERIMENTS}

The relatively simple shape of the nonlinearity obtained in the last section makes it tempting to try computer experiments with similar equations. We started with $g=-\alpha \sinh (\alpha>0)$. For $\alpha<\pi / 2$ and sufficiently close to $\pi / 2$, this equation has an unstable slowly oscillating periodic solution $y^{\alpha}$ of Kaplan-Yorke (KY) type; see, e.g., [ILW]. (Note that $-\sinh \notin B C^{1}(\mathbb{R}, \mathbb{R})$, but the values of the feedback function 
far away from the range of the periodic solution are irrelevant.) These solutions form a branch that bifurcates backwards from the zero solution at $\alpha=\pi / 2$.

Initial segments $y_{0}^{\alpha}$ of periodic solutions can be calculated from an associated system of ordinary differential equations in the plane, compare, e.g., [DL-W]. We used the parameter value $\alpha \approx 1.38$, where the amplitude of the KY-solution is approximately 1 .

We approximated segments in the unstable manifold of $y^{\alpha}$ simply by starting with a slight perturbation of $y_{0}^{\alpha}$ and computing the forward solution. As long as this solution remains close to the orbit of $y^{\alpha}$, one expects that its segments will approach the unstable manifold exponentially, since $y^{\alpha}$ is hyperbolic.

Then we tried to modify the function $-\alpha$ sinh along the lines of the construction from the previous sections, with the aim to obtain (approximately) homoclinic behavior for solutions starting (approximately) in the unstable manifold of $y^{\alpha}$. It turned out that one does not need the type of modification which was denoted $g_{1}$ in this paper, because $-\alpha \sinh (x)$ is already decreasing steeply enough for positive values of $x$.

We therefore only changed $-\alpha \sinh$ for negative values of $x$ such that the new function has one single maximum at some negative $x$. In fact, we found approximately homoclinic behavior for the following nonlinearity, which is $C^{\infty}$ and has precisely one maximum (as can be seen from elementary curve discussion).

$$
\begin{gathered}
g(x):=-\alpha \sinh (x) \cdot \Phi(x), \quad \text { where } \alpha \approx 1.38, \text { and } \\
1, \quad x \geq-1.3, \\
\Phi(x):=\left\{\begin{array}{rl}
1 & x<-1.3 . \\
\frac{1}{1+10 e^{-x} \cdot e^{1 /(x+1.3)}}, & x
\end{array}\right.
\end{gathered}
$$

Due to the backward bifurcation, the zero solution is stable for our $g$. The main numerical observation that we want to report is the following:

It seems that for arbitrary initial segments, the forward numerical solution converges to zero. Typical numerical solutions spend little time, if any, in the vicinity of the unstable KY-solution $y^{\alpha}$ and of the numerically homoclinic solution.

We should mention that, contrary to the previous work [DL-W], we have not put much emphasis on numerical accuracy in these experiments. In fact, an Euler method was used to compute solutions. Based on numerical experience, we believe that, in this case, higher numerical precision would qualitatively give the same results.

The conclusion from the computations is that the 'chaos', the existence of which we have analytically proved, may be hard to observe in experiments with similar nonlinearities. 


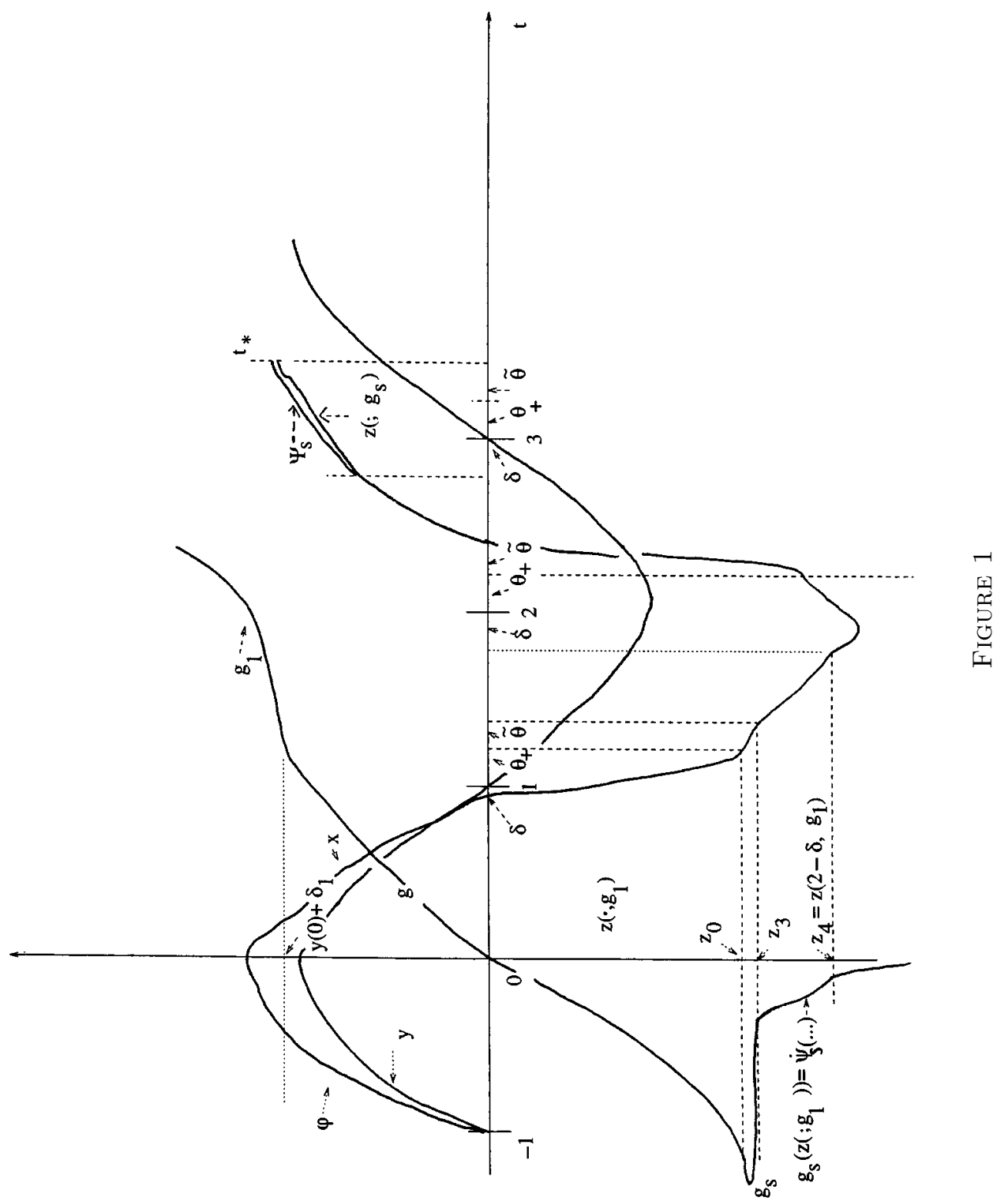




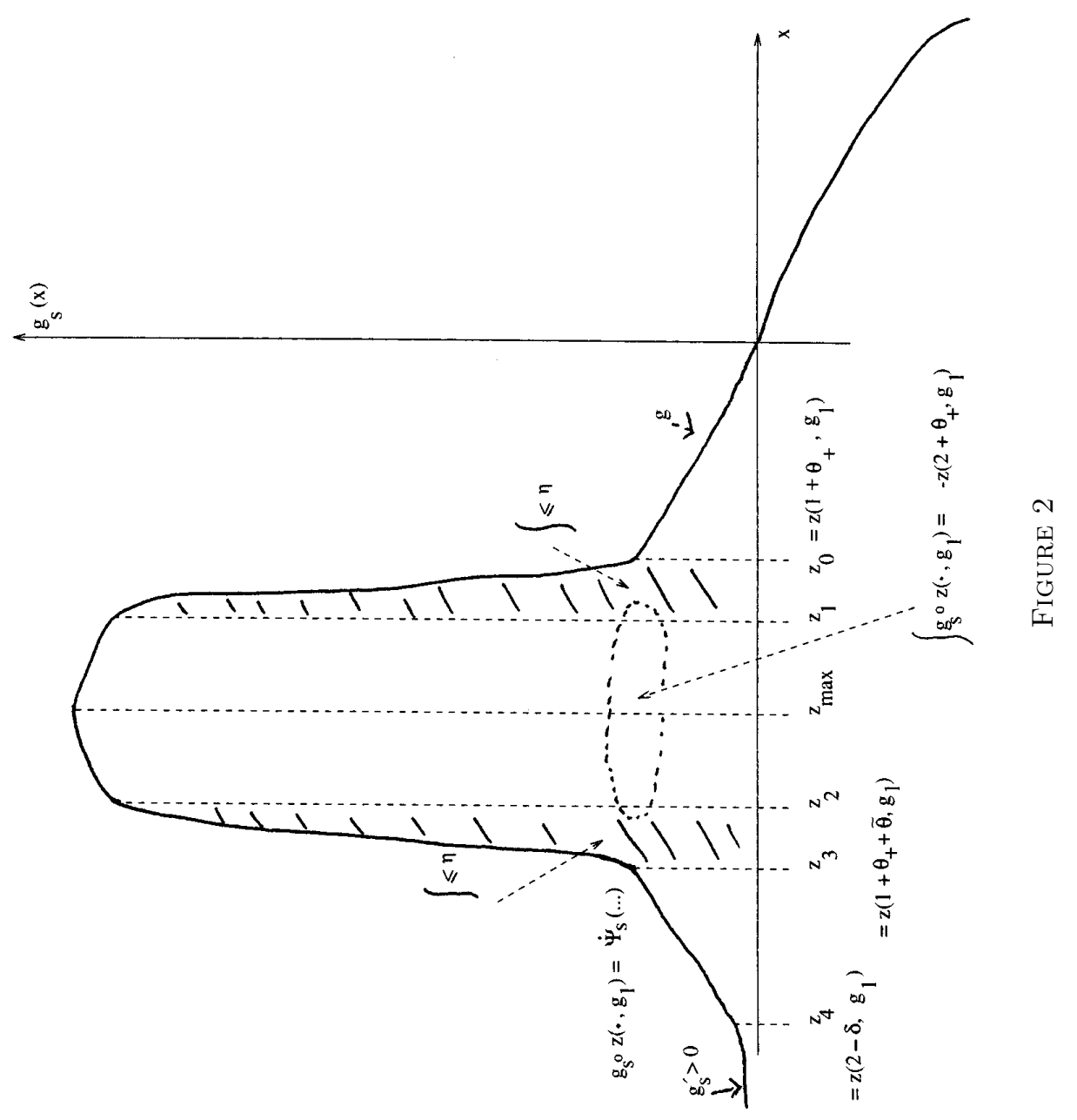




\section{REFERENCES}

[AdHW] U. An der Heiden and H.-O. Walther, Existence of chaos in control systems with delayed feedback, J. Diff. Equs. 47 (1983), 273-295. MR 85a:58061

[BC1] M. Benedicks and L. Carleson, On iterations of $1-a x^{2}$ on $(-1,1)$, Ann. of Math. 122 (1985), 1-25. MR 87c:58058

[BC2] M. Benedicks and L. Carleson, Dynamics of the Hénon map, Ann. of Math. 133 (1991), 73-169. MR 92d:58116

[DL-W] P. Dormayer and B. Lani-Wayda, Floquet multipliers and secondary bifurcations in functional differential equations: Numerical and analytical results, Z. Angew. Math. Phys. (ZAMP) 46 (1995), 823-858. MR 97f:34047

[Dr] R.D. Driver, Ordinary and delay differential equations, (Applied Mathematical Sciences 20) Springer Verlag, New York, 1977. MR 57:16897

[DvGV-LW] O. Diekmann, S.A. van Gils, S.M. Verduyn-Lunel, and H.-O. Walther, Delay Equations, (Applied Mathematical Sciences 110) Springer-Verlag, New York, 1995. MR 97a:34001

[G] T. Gedeon, Cyclic feedback systems, Mem. AMS (to appear). CMP 97:07

[GP] P. Grassberger and I. Procaccia, Measuring the strangeness of strange attractors, Physica 9D (1983), 189-208. MR 85i:58071

[H] J.K. Hale, Functional Differential Equations, (Applied Mathematical Sciences 3), Springer Verlag, New York, 1971. MR 57:6711

[HL1] J.K. Hale and X.B. Lin, Symbolic dynamics and nonlinear semiflows, Ann. Mat. Pura Appl. (4) 144 (1986), 229-259. MR 89g:58130

[HL2] J.K. Hale and X.B. Lin, Examples of transverse homoclinic orbits in delay equations, Nonlinear Analysis 10 (1986), 693-709. MR 87i:34087

[HS] J.K. Hale and N. Sternberg, Onset of Chaos in Differential Delay Equations, J. Computational Phys. 77 No.1 (1988), 221-239. MR 89h:58115

[HV-L] J.K. Hale and S.M. Verduyn-Lunel, Introduction to Functional Differential Equations, (Applied Mathematical Sciences 99), Springer Verlag, New York, 1993. MR 94m:34169

[ILW] A. Ivanov, B. Lani-Wayda and H.-O. Walther, Unstable hyperbolic periodic solutions of differential delay equations, Recent Trends in Differential Equations, ed. R.P. Agarwal, World Scientific, Singapore, 1992, pp. 301-316. MR 93h:34125

[KS] U. Kirchgraber and D. Stoffer, Chaotic behavior in simple dynamical systems, SIAM Review 32 No. 3 (1990), 424-452. MR 91e:58141

[KY] J.L. Kaplan and J.A. Yorke, On the stability of a periodic solution of a differential delay equation, SIAM J. Math. Analysis 6 (1975), 268-282. MR 50i:13812

[L-W1] B. Lani-Wayda, Persistence of Poincaré mappings in functional differential equations (with application to structural stability of complicated behavior), J. Dyn. Diff. Equs. 7 No. 1 (1995), 1-71. MR 96e:34118

[L-W2] B. Lani-Wayda, Hyperbolic Sets, Shadowing and Persistence for Noninvertible Mappings in Banach spaces, Research Notes in Mathematics No. 334, Longman Group Ltd., Harlow, Essex, 1995. CMP 98:01

[L-WW1] B. Lani-Wayda and H.-O. Walther, Chaotic motion generated by delayed negative feedback, Part I: A transversality criterion, Diff. Int. Equs. 8 No. 6 (1995), 1407-52. MR 96c: 58115

[L-WW2] B. Lani-Wayda, and H.-O. Walther, Chaotic motion generated by delayed negative feedback, Part II: Construction of nonlinearities, Math. Nachr. 180 (1996), 141-211. MR 97g:58147

[La] A. Lasota, Ergodic problems in biology, Asterisque 50 (1977), 239-250. MR 58:9378

[Laz] V.A. Lazutkin, Positive Entropy for the Standard Map I, Preprint 94-47, Université de Paris-Sud, Mathématiques, Bâtiment 425, 91405 Orsay, France, 1994.

[LWCz] A. Lasota and M. Wazewska-Czyzewska, Matematyczne problemy dynamiki ukladu krwinek czerwonych, Mat. Stosowana 6 (1976), 23-40.

[LY] T.Y. Li and J.A. Yorke, Period three implies chaos, Am. Math. Monthly 82 (1977), 985-992. MR 52:5898

[MG] M.C. Mackey and L. Glass, Oscillation and chaos in physiological control systems, Science 197 (1977), 287-295. 
[M] M. Morse, A one-to-one representation of geodesics on a surface of negative curvature, Am. J. Math. 43 (1921), 33-51.

[MH] M. Morse and G. Hedlund, Symbolic Dynamics, Am. J. Math. 60 (1938), 815-866.

[M-P] J. Mallet-Paret, Morse decompositions for delay differential equations, J. Diff. Equs. 72 (1988), 270-315. MR 89m:58182

[M-PS] J. Mallet-Paret and G. Sell, The Poincaré-Bendixson theorem for monotone cyclic feedback systems with delay, J. Diff. Eqns. 125 (1996), 441-489. MR 97a:34193b

[M-PSm] J. Mallet-Paret and H.L. Smith, The Poincaré-Bendixson theorem for monotone cyclic feedback systems, J. Dyn. Diff. Equs. 2 (1990), 367-421. MR 91k:58098

[M-PW] J. Mallet-Paret and H.-O. Walther, Rapid oscillations are rare in scalar systems governed by monotone negative feedback with a time lag, Preprint, Math. Inst. Univ. Giessen, 1994.

[P] K.J. Palmer, Exponential dichotomies, the shadowing lemma and transversal homoclinic points, U. Kirchgraber and H.-O. Walther (eds.), Dynamics Reported, vol. I, Teubner-Wiley, Stuttgart/Chichester, 1988, pp. 265-306. MR 89j:58060

[S] S. Smale, Differentiable Dynamical Systems, Bull. AMS 73 (1967), 747-817. MR $\mathbf{3 7 : 3 5 9 8}$

[SW] H. Steinlein and H.-O. Walther, Hyperbolic Sets, Transversal Homoclinic Trajectories, and Symbolic Dynamics for $C^{1}-$ maps in Banach Spaces, J. Dyn. Diff. Equs. 2 (1990), 325-365. MR 92b:58170

[W1] H.-O. Walther, Homoclinic solution and chaos in $\dot{x}(t)=f(x(t-1))$, Nonlinear Analysis 5 (1981), 775-788. MR 83a:58060

[W2] H.-O. Walther, Hyperbolic periodic solutions, heteroclinic connections and transversal homoclinic points in autonomous differential delay equations, Memoirs AMS 402 (1989). MR 90m:58184

[W3] H.-O. Walther, The 2-dimensional attractor of $x^{\prime}(t)=-\mu x(t)+f(x(t-1))$, Memoirs AMS 544 (1995). MR 95f:58070

Mathematisches Institut der Universität Giessen, Arndtstr. 2, 35392 Giessen, GerMANY

E-mail address: Bernhard.Lani-Wayda@math.uni-giessen.de 